\title{
Understanding common sense themes of Intellectual and Creative Work: The social representation of Intellectual Property
}

by

Joel, Wiramu. Pauling

A thesis

submitted to the Victoria University of Wellington in fulfilment of the requirements for the degree of

Master of Science in Psychology.

Victoria University of Wellington 2009 


\begin{abstract}
This thesis examines the construction of Intellectual Property discourses using the social Psychological theoretical framework of Social Representations theory (Moscovici, 1984), and explores the various themes which emerge around the treatment of knowledge, ideas and creative work from both historical and contemporary perspectives. The first two chapters introduce Social Representations theory and the methodology of Thematic Analysis. Study one presents a historical account and literature review on the general themes of knowledge, intellectual and creative endeavours, and how various cultures and social powers have approached these concepts throughout history. It includes an overview of current technological and social changes around the same themes, the challenges these changes may have on existing social representations, and the groups that have vested interests in particular representations. The idea that existing dominant representations cannot adequately incorporate new representations arising from users and adopters utilising the new medium of the 'network' as a social-cultural tool is also introduced. Study two examines similar topics through analysis of public submissions to the New Zealand Patent act review. In this study, individual submissions are analysed in detail using a thematic analysis-like process, incorporating this into a Social representations framework designed to extend and test the representations observed in study one. Evidence of a dominant industrial representation involving market economic treatment of knowledge centred on a physical resource conceptual anchor was observed. Conflicting social representations held by other groups included representations of a collectivist common good centred on innovation and rights themes. Evidence that significant re-representation of the property conception away from a physical good anchor by various groups was found. Social, cultural, and economic consequences of these competing representations effect on societies are considered.
\end{abstract}




\section{Acknowledgements}

Thanks to current and past flats. 26 Palmer Street, Neil, Chris, and Dane you have been saviours coping with my destitution upon returning from my great overseas adventures and providing shelter and food. The same goes to the West Rd flat and Mike, Derek and Rohan whom have provided the same deal ( but with slightly less and more intermittent Internet, and far more distractions). The computer science graduate group AKA the Peoples Republic of Memphis, similarly your interest, shared fridge and comfy couches have been a great benefit. To my supervisor Jim Liu for putting your trust in me to look after your house for 6 months, and for sticking up for me on occasion, and providing the signposts I needed along the way to keep me somehwat cohrent. Marc Wilson for proving a stand in for Jim, and being willing to try some of my nonpsychology nomative methods of document development on occasion. To all the people whom I met in my travels through couchsurfing and helped me gather data from tech malls around south east Asia, that ultimately I did not end up using. Yayi whom has had to deal with my ability to give a straight answer on anything the last 6 months, and whom my constant pre-occupation/anxiety and grumpiness has had the greatest toll. My laptop, why? Because it has not once lost a single word of this text, nor forced me to use any of the 3 or 4 different mechanisms I devised to keep copies available. To all the various Internet communities discussion boards, focus groups, lawyers, techies, geeks and businesses and academics I have discussed this topic with over the last 3 years, your time and interest and activities has kept me churning the ideas over. Aaron for providing late night pick ups from the University. The efforts of Digital archivists and organisations throughout the world who are digitising and 
making available cultural heretidge and knowledge resources in a Usefull fashion. The advances in Digital Document search and retrieval such as Google books during the last 2 years has been of immense benefit to this research research, and archival efforts of the Gutenburg project and Wikisource have been equally appreciated, (academic journal publishers conversly are still lagging on this front, and more a source of frustration than utility). Anyone else who has suffered from my general anxiousness and pre-occupation along the way that has led me to ignore a lot of what is going on around me in the vain attempt of not becoming distracted. And lastly Charmaine whom gave me the impetus to start this in the first place, despite your not being here for any of this, this thesis remains largely a symbol of your ability to motivate me to do things I otherwise might not. 


\section{Contents}

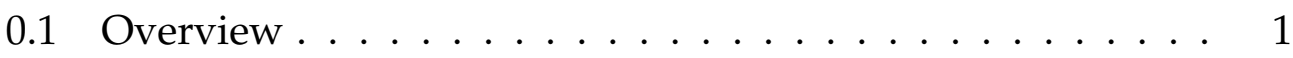

0.1 Structure .................... 5

1 Social Representations Theory $\quad 7$

1.1 History of Social Representations . . . . . . . . . . . . . . . . . . . . . . .

1.2 Common Sense . . . . . . . . . . . . . . . . . . . 11

1.2.1 A Legal Example: Human Rights Violations . . . . 15

1.2 .2 Social Objects . . . . . . . . . . . . . 17

1.3 Institutions and Groups . . . . . . . . . . . . . . . . 19

1.4 Themata . . . . . . . . . . . . . . . . . 20

1.4.1 Taxonomies ................... 21

1.4.2 Contradiction Accommodation . . . . . . . . . . . 21

1.5 Process model or Sociogenesis of Social Representations . . . 22

$1.5 .1 \quad$ Brute Facts . . . . . . . . . . . . . . . 25

1.5.2 Collective Symbolic Coping . . . . . . . . . . . . 26

1.5 .3 Consensus . . . . . . . . . . . . . . 27

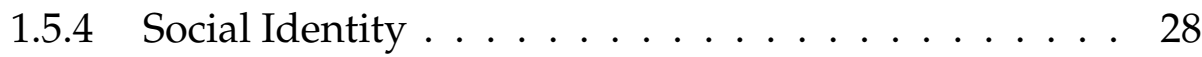

1.6 Anchoring and Objectification . . . . . . . . . . . . . . 30

1.6.1 Making the unfamiliar, familiar . . . . . . . . . . 31

1.7 Metaphors and Mechanisms . . . . . . . . . . . . . . 33

1.8 Summary . . . . . . . . . . . . . . . . . 34

2 Methodologies $\quad 35$

2.1 Thematic Analysis . . . . . . . . . . . . . . . 36

2.1.1 General Method of Thematic Analysis . . . . . . . . . 37

2.2 The Corpus . . . . . . . . . . . . . . . . . . 41

2.2.1 Historical Thema . . . . . . . . . . . . . . . . 41 
2.2.2 The Patent Act 1953, 2004 review: Public Submissions 41

2.2.3 Internet articles and discussions . . . . . . . . . . 43

2.3 Summary recap of research goals . . . . . . . . . . . . . 45

3 The Story of ICW and Society 46

3.1 Toward social representations of knowledge . . . . . . . 50

3.1.1 Early Conceptions of Knowledge . . . . . . . . . . . . 50

3.1.2 Comments on the treatment of knowledge in prehistory ....................... 53

3.2 Early modern era and the advent of print . . . . . . . . . . 54

3.2.1 First attempts attempts at commodification of knowledge ...................... 54

3.2.2 Incorporating diffuseness into the SR of ICW? . . . . 56

3.2.3 The division of knowledge in Europe and the influence of Martin Luther . . . . . . . . . . . . . 57

3.3 Interpreting privilege and Monopoly . . . . . . . . . . . . 60

3.3 .1 Of the Author . . . . . . . . . . . . . . . . . . 60

3.4 The trinity of Representation: User, Creator and Proprietor . 64

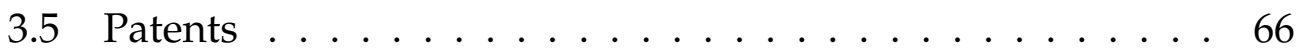

3.6 Setting a standard for monopolies . . . . . . . . . . . 68

3.7 The Modern Era . . . . . . . . . . . . . . . . . . . . . . . . . . 69

3.8 Solidifying Institutional Legitimacy of Property Discourses . 75

3.9 Conclusion of the modern era . . . . . . . . . . . . . 77

3.10 The advent of digital media and the post modern age . . . . 79

3.11 Patents and Copyright in the Media . . . . . . . . . . . 82

3.12 Concluding remarks and the map of historical representations 83

4 The New Zealand Patent Act review $\quad 85$

4.1 "Intellectual Property" as Legal Thema . . . . . . . . . . . . 86

4.1.1 A mathematical metaphor of thought and knowledge 88

4.1.2 Difficulties in the thema of Intellectual Property . . . 89

4.1.3 Groups and trends in relation to legal thema . . . . . 92

4.1.4 Revisiting legal reified definitions . . . . . . . . . . . 94

4.2 The legislative history in New Zealand . . . . . . . . . . . 97

4.3 Specific New Zealand issues: Treaty of Waitangi . . . . . . . 98 
4.4 The Data Set . . . . . . . . . . . . . . . . . . . . . . 100

4.4.1 Thematic Extraction process: Tagging . . . . . . . . 103

4.4.2 On overall Positive, Negative or integrated perspectives and consensus . . . . . . . . . . . . . . 104

4.5 Extracted Themes . . . . . . . . . . . . . . . . . 105

4.5.1 International Obligations . . . . . . . . . . 106

4.5.2 Business, Economics and Prosperity . . . . . . . . . . 110

4.5.3 Property, Exclusions and Control . . . . . . . . . . . . 114

4.5.4 Human Rights, Traditional Knowledge and The Public120

4.5.5 Authority and Legitimacy . . . . . . . . . . . . . . 125

4.5.6 Inventor, Novelty and Innovation . . . . . . . . . . . 129

4.5.7 Drafting Issues / Legal wording . . . . . . . . . . . . . . . . . . . . . . . . . . .

4.6 On the role of conflict and contradictions . . . . . . . . . . . 131

4.7 So where is the Property? Summary . . . . . . . . . . . . . . 133

4.8 Informing Social Representations Theory . . . . . . . . . 135

5 Review and Concluding remarks $\quad 137$

5.1 Concluding remarks . . . . . . . . . . . . . . 137

5.2 Final Thought . . . . . . . . . . . . . . . . . 143

$\begin{array}{ll}\text { Bibliography } & 144\end{array}$ 


\subsection{Overview}

Understanding knowledge, the essence of thought, communication and dialogue is (and will continue to be) at the heart of a diverse range of both academic and societal pursuits. In this regard, this thesis is intended to expand recent discussions occurring in society centred on the advancing technological and economic changes that are occurring in the world (Pugatch \& Elgar, 2006). Consequences of increasing globalisation and the decreasing communicative distances between any two entities, as enabled by information and communication technologies (ICT). The discussion of knowledge continues to be central to these changes. However, understanding the 'common sense' about knowledge and information exchange has largely gone ignored in research. Instead, the focus has been on economic and legal challenges, and societies and individuals outside of this scope have generally been treated as passive recipients at the end of value chain. The structures of the recent past have been shaped by mass media and the technologies that enabled it. This has meant access was guarded by 'gatekeepers of money', and overt control and censorship either via government or that of the market. Today's technological environment challenges these traditional structures. Every individual is able to gain access to technology allowing for similar distributive power as once reserved for mass media, and that only a decade ago was impossible. Through the medium of the network, societies and minority groups have found a new empowerment that facilitates access to a participatory knowledge environment once reserved for the elite. This has led to significant changes in the flow of abstract knowledge in society. The chance that a piece of information around you right now has come from a peer driven distribution system, as opposed to a centrally managed one, is far greater than it was 15, 10 or even five years ago. Whether it is a song you are listening to on your media device, a print on a t-shirt, or an image on your wall. Most important is the idea in your head, from an on-line journal, an article from an indynews website, a political blog, or a recipe for designing plastic explosives. The elements that make up your thought and ideas are increasingly likely to come to you from sources detached from organisations with the distributive powers of the past. It is also more likely that 
you transmit information to others outside of your physical social network in the same way, knowingly or not.

The legal-economic nexus that has grown up around the value of exclusive rights associated with knowledge, and the transmission and communication of the same, has difficulty harnessing these new participatory practices. Their representation of knowledge is fundamentally different, where previously knowledge has been an item of trade that has leverage in a variety of markets. For the individual the same bits of knowledge, and the processes surrounding it, fulfil a very different function. The same can be said for governments, industry and creators. All share different representations around how any given bit of knowledge came to be, will function now and in the future, and how it should be controlled. Because of inherent circumstantiality, and because knowledge is an intangible and abstract conception to begin with, treating knowledge from any one viewpoint comes, by definition, at the exclusion of all others. One cannot treat it as a commodity while also agreeing that it can have no objective value. Conversely, one cannot attach commodity rights and legal considerations to every piece of information and thought that you experience. So, to answer the question of control for one group is at the exclusion of others. Discussions around these themes are the ones which are resonating around the globe at the moment, including issues of fair use, industrial application, commercial exploitation, creative expression, open access, all are seeded within how different groups conceive of knowledge information and the processes around it, and how best to control it. The questions of control, and the rights associated with knowledge distribution use and creation are not new. The changes in technology and how global economies and many organisations now position themselves to rely on particular representations of knowledge, and more importantly its exchange, are echoes of past debates around similar themes spurred by comparable technological and societal changes.

This thesis will map the history of, and current discussions about, the representations of the processes and conceptions surrounding knowledge. I shall attempt to demonstrate that there are distinct representations of abstract knowledge processes, which for simplicity I will refer to as Intel- 
lectual and Creative Work (ICW) throughout the thesis, in different parts of society, and that these representations can serve different, potentially conflicting, functions.

The topics covered here represent a work in progress, a moving target, and many of the branches of the original thesis planned early in 2004 have not received the time they deserve to fully cover this fundamentally important social and cultural evolution. This manuscript represents a small contribution to the global developments and body of study that is rapidly emerging around these issues. Since the original thesis was conceived, many of the references have become outdated, or at least in need of clarification and expansion, and the research that I have pooled around this area has been constant, if unstructured. There is data from side studies that I carried out while travelling Asia that have not been included, huge topic areas. One such involves developments in the United States around proposed orphaned works bills, and a particularly relevant legal case that may see the overturning of the 1984 legal precedent establishing the legal basis for Software and Business methods patents (not to mention recent local developments in copyright bill amendments, digital strategy bills, WAI262 among others) that just have not found a place to be incorporated. Special note must be made of the psychological theories applied here while Social Representations theory In the end has been the correct choice as the principle theory of investigation, there are other equally relevant theories of inter-group conflict, generalised political psychology, authority and legitimacy, motivation, altruism and image management, organisational and industrial psychology, leadership and community formation, and many more from the wider discipline, that might also be applied to the subject matter of this investigation.

This particularly abstract and difficult to 'nail down' topic will only continue to grow in relevance in the future. At this point in time we have very clear choices about how we can proceed, making choices about the representations we choose to privilege, and legitimise, as a society. It is only hoped that this thesis can inform those decisions by revealing the commonly encountered conceptions to this point in history. 
Overall Aim The aim of the thesis is to demonstrate current societal trends in debate around how knowledge artefacts are appropriated into legitimised representational fields, and to do so in a way that conforms to the processes involved in the generation of Social Representations. The current surge in discourse around the topic suggests a form of symbolic group coping at societal and cultural levels. Further evidence to support this comes from the conflict and integration of new phenomena, and information relating to various positions, by disparate groups. The goal of the thesis is to try to capture, and provide plausible explanations for, how these conflicting representations contribute to a common sense understanding of the various group based representations of intellectual property, and also how each group-specific representation differs from others. A secondary goal is to determine if a reified legal political-economic nexus representation exists, and if it functions as the dominant common sense understanding. If this dominant representation exists how, and to what extent, is it included in everyday discourse. The adoption of thematic analytic methodologies to achieve these goals within the greater theoretical framework of Social Representations theory (SR) (Moscovici, 2001; Wagner \& Hayes, 2005) hopefully will lead to a better understanding of the component themes of these representations, and how these differing representations may influence behaviour and stated attitudes (Wagner et al., 1999). In achieving these goals, there is a natural progression towards producing a narrative history in the form of thematic maps that illustrate both historical and present-day representational fields. In fact, production of such maps is a key goal of this work.

It is my hope that my consideration pf the general topic itself spurs further research within the wider field of psychology as to the real-life treatment of knowledge artefacts in day-to-day life, as legal and economic perspectives are vastly over-represented within the literature.

Research Methodology My research methodology employs Thematic Analysis (Aronson, 1994; Braun \& Clarke, 2006) in a theoretically-driven approach with Social Representations as a guiding theory, it is therefore necessarily constructionist in nature. The data corpus comprises generally 
of public discourse on topics relating to Intellectual Property. The main dataset used from this corpus is that of public submissions to the New Zealand Patent Act review 2002 - 2004. Thematic analysis is employed to discern key themes present in this dataset. A wider corpus of Internet news and discussion forum articles will be used to provide corollaries or additional themes in relevant topic areas. For a full description of the methods used, please refer to Methodology section which begins on page 35.

The bulk of the opening chapters provides an introduction to Social Representations Theory followed by an extensive background reading of historically relevant literature relating to the control and conception of what I call ICW. The importance of creating a historical context in which to frame current trends and discourses is the assistance it provides in framing and influencing current themes and discussion. Within SRT this involves the processes referred to as anchoring and objectification, that in turn relate to symbolic coping. These concepts are discussed further in 1 . Key to this thesis is the aspect of conflict associated with this process and the debate. Patents are used throughout, as they represent a particularly succinct legal construct, and one that elicits these conflicting positions. Debate surrounding other intellectual property topics will be used where appropriate to illustrate themes relevant outside of patent law discourse. Of particular note is copyright law, which arguably has produced even more conflicting discourse than patent law.

\subsubsection{Structure}

As mentioned previously, the introduction section consists of a chapter providing the reader with a theoretical overview of social representations theory. This is particularly important in establishing the core concepts involved, as they will be referred to throughout. Thematic analysis is also briefly introduced as the basis for the methodology used in analysis. A large section is devoted to a review of historical events and accounts loosely around control and construction of ICW. Much of this is devoted to understanding how various treatments of knowledge throughout history including that of; religion, culture, technological advance, the state, 
censorship, have (among others) been implicated in constructing representations around ICW. This section relies on historical narrative working toward an historical representational map of the subject matter. This broader historical frame allows for the extraction of likely themes, and this is used as an initial thematic map for an interpretive analysis of the corpus. The initial thematic map used as a starting point for themes can be observed in 2.1.1 on 40 of the methodologies chapter. Discussion of each theme occurs alongside analysis within the Patent submission chapter. Implications of this analysis include consideration of how each of the identified themes may relate to building a representational map of common sense understanding of ICW. The product of these themes as an inter-related thematic and representational map is investigated from a 'highlighting and hiding' (Lakoff \& Johnson, 1980a) approach. Future research directions on the topic, and how it applies to Industry and Society in general, will be discussed. 


\section{Chapter 1}

\section{Social Representations Theory}

\footnotetext{
"The power of narrativium in Roundworld is immense. Things happen because of it that you would never expect from the laws of nature. For example, the laws of nature pretty much forbid an Earthbound object suddenly leaping up into space and landing on the Moon. They don't say it's impossible, but they do imply that you could wait a very long time indeed before it happened. Despite this, there is a machine on the Moon. Several. They all used to be down here. They are there because, centuries ago, people told each other romantic tales about the Moon. She was a goddess, who looked down on us. When full, she caused werewolves to change from humans into animals. Even then, humans were quite good at doublethink; the Moon was clearly a big silver disc, but, at the same time, she was a goddess.

Slowly those tales changed. Now the Moon was another world, and by harnessing the power of swans we could fly there in a chariot. Then (Jules Verne suggested) we could get there in a hollowed-out cylinder fired by a giant gun, located in Florida. Finally, in the 1960s, we found the right kind of swan (liquid oxygen and hydrogen) and the right kind of chariot (several million tons of metal) and we flew to the Moon. In a hollowed-out cylinder, launched from Florida. It wasn't exactly a gun. Well, actually it was in a basic physical sense; the rocket was the gun and it went along for the ride, firing burnt fuel in place of a bullet." - The Science of Discworld II: the Globe Pratchett, Stewart, and Cohen (2003, pg40)
}

Social Representations are comprised of a set of structured components which build on a number of philosophical, sociological and anthropological traditions (Voelklein \& Howarth, 2005). They are incorporated within psychology under the social cognition umbrella (e.g., Augoustinos, Walker, \& Donaghue, 2006) by some and into the growing discursive tradition (e.g., Antaki, Billig, Edwards, \& Potter, 2003) by others, and have been incorporated somewhat in the more dominant experimental/cognitive positivist tradition by others (e.g., Bauer \& Gaskell, 1999). Moscovici, 1984 as cited in Moscovici, 1988 provides the following definition of social representations:

... the collective elaboration "of a social object by the community for the purpose of behaving and communicating". They are further referred to as "system of values, ideas and practises with a twofold function; first, to establish an order which will enable individuals to orientate themselves in their material and social world and to master it; and secondly to enable communication to take place among the 
members of a community by providing them with a code for social exchange and a code for naming and classifying unambiguously the various aspects of their world and their individual and group history". - Moscovici, 1988

Which background apologists of the theory subscribe to greatly influences the methodologies employed in their investigation, and, to an extent, the various components of the theory that are employed (Verheggen \& Baerveldt, 2007). This comes both as a blessing and a bane for researchers using the theory, as there is a certain detachment from any one tradition. Some, however, argue for a more nomothetic approach. For example, in their paper Discourse analysis means doing analysis Potter and Edwards (1999) argue towards using Discourse Analysis (DA) as a research methodology, because SR lacks methodological focus. They reason that SR contains elements of both representations (in concepts such as social objects) such as used in DA, and also cognitive-perceptual personal constructs (such as in categorising and sense making). This common thread between discourse analysis and SRT and other socially grounded theories very much overlap from one another in there high level approach in their representational analysis. For example social representations theory includes components of classical Durkheimian (Durkheim, 1973) sociological theory, and linguistic theory elements such as semiotics, metaphor analysis (Lakoff \& Johnson, 1980b) and draws heavily from the Sapirwhorf tradition of social constructivism expressed via linguistic relativity hypothesis (Sapir, 1963; Sapir-Whorf hypothesis - Wikipedia, the free encyclopedia, n.d.; Lakoff \& Johnson, 1980c). In this vien it posits that a cultures linguistic and gramatical portfolio influences how members interact with the world around them. The level at which a purely linguistic approach differs from these commonalities with SRT is that SRT is almost a purely group interaction focused theory, whereby language portfolios demarcate groups, and it is the group associations that influence behaviour of individuals rather than language portfolios in and of themselves. This relationship between the Sapir-Whorf tradition is one where both share a structuralist view of reality, however in SRT there is a focus on how the mediating factor of the social environment affects individual perception 
of reality.

For these reasons, and others, SRT is often overlooked within Psychology due to a disciplinary bias towards experimental methodology and the cognitive positive tradition that accompanies it (Jost \& Kruglanski, 2002). Because SRT contains elements consistent with both a discursive/representational perspective and a cognitive one, it draws much criticism from both.

\subsection{History of Social Representations}

The history of the original theory stems from Moscovici's 1961 (Moscovici, 1984) investigation of French public conceptions about the practise of psychoanalysis (Voelklein \& Howarth, 2005). Moscovici argued that Emile Durkheim's theory of collective representations provided too general an approach to how particular domains were influential in forming a collective representation. Durkheim broadly introduces conceptual categories of what constitutes any given representation by providing cognitive categories such as; - science, ideology, worldview and myth - cited in (Voelklein \& Howarth, 2005). The distinction between these conceptual/cognitive categories however is never defined adequately for Moscovici.

The main problem emphasised by Moscovici in his original 1961 piece is that within a Durkheimian collective representation framework, representations were essentially transmitted in an asynchronous fashion. This means that institutions provided the principle representations for any given society, and that any given representation was inherently resistant to change once transmitted (Voelklein \& Howarth, 2005). The homogeneous and referentially authoritative concepts such as those in Durkheimian collective representations however did not adequately describe the diverse range of the data Moscovici had collected and analysed, from a broad number of both quantitative and qualitative approaches, with content analysis (Billig, 1991) being one of the more important. Moscovici observed a number of changes and variations in the representations being considered. It was clear that there were interactions and diverging representations within a given society. By labelling representations at a societal level, this hetero- 
geneity was lost and worse, inadequately described the processes occurring. Moscovici posited that there were ongoing processes within societies which influenced the emergence of representations. While institutions and media were the primary influence on the ongoing social processes, intra-society communications were observed to play a more active role than was considered in Durkheim's conception (Moscovici, 1984). These inter-societal institutional representations were named Social Representations, as they were observed to require the social participation of a members of any given group in a society for the representations to exist and be maintained (Verheggen \& Baerveldt, 2007). This basic account embedded in Moscovici's original introduction of social representations provides the beginning point for what has today become a rich epistemology of knowledge surrounding the process whereby Social Representations are both identified and the processes involved in formation, maintenance and transmission (Verheggen \& Baerveldt, 2007). The varying ways in which Social representations have been redacted in terms of how intricacies of SR theory are employed by researchers has been a point of contention (Verheggen \& Baerveldt, 2007; Potter \& Billig, 1992; R. M. Farr, 1993). Further complicating this are the fundamental issues arising because of the theory's agnostic nature in regard to methodology. Lacking a particular research methodology leaves it open to the same criticisms of (for instance) Grounded Theory (Holloway \& Todres, 2003), namely that it lacks objectively identifiable features and relies too heavily on subjective/reflexive influence of researchers own internal cognitive processes. (Boyatzis, 1998; Braun \& Clarke, 2006)

Moscovici has maintained a presence within the evolution and dialogue of the theory itself, facing heavy criticism over explanations and accounts, while writing at length. A major criticism levelled against Moscovici (Verheggen \& Baerveldt, 2007) is failure to adequately explain what is sometimes referred to as the core (Bauer \& Gaskell, 1999) presuppositions of the theory. Most of these criticisms are aimed at what is referred to as ambiguous (Räty \& Snellman, 1992; Markova, 2000) explanations.

Wagner and others (Wagner et al., 1999) have been key participants in the evolution and critique of social representations theory, and while 
many scholars (Verheggen \& Baerveldt, 2007; L. Liu, 2004; Voelklein \& Howarth, 2005; Wagner, 1995) utilise concepts defined in Wagner et al. (1999) process/sociogenesis model of social representations (Wagner et al., 1999). There is a more fully integrative view of SR theory as expressed in the wider literature. The various minutiae defined by Wagner and others (Wagner et al., 1999) are for the most part have been incorporated in research largely unchallenged, save for variations in divergent methodological considerations (Voelklein \& Howarth, 2005). This is in contrast to those researchers employing SRT concepts outside of Wagner's reading, which has created a core and periphery dichotomy in SRT.

To this end relying primarily on the concepts defined by Wagner et al. (1999) and a recent reviews (Verheggen \& Baerveldt, 2007; Voelklein \& Howarth, 2005) of dialogues in Social Representations Theory provides the most accessible break down of Social Representations. Thus the emphasis on the concepts in what Verheggen and Baerveldt (2007) call Wagner's epistemology, which views the generation of Social Representations as recursive sequence of events surrounding collective coping with new information, will be apparent to any reader familiar with SR theory. Some distance is kept from more experimentally grounded approaches such as Bauer and Gaskell (1999) and R. Farr (1987).

The biggest challenge in explaining SRT is that it has a very reified set of concepts, all which assume familiarity with the others. Explaining each of these in turn becomes a difficult task. It is left to the reader to anticipate this and if terms appear before they are defined that it is a deliberate attempt to encourage further exploration. Likewise if a term is explored from multiple perspectives, as is done with the explanation of common sense, then it is these multi-referential perspectives which help define the concept because of its abstract nature.

\subsection{Common Sense}

Central in dialogues (Voelklein \& Howarth, 2005; Markova, 2000; Moloney \& Walker, 2002) is the notion of social representations drawing on 'common sense' knowledge. Common sense knowledge when viewed within a 
SR context is knowledge that is widely shared and held to be true by most, if not all, members of a given society or community. Moreover, common sense is taken by many researchers as implicitly providing the basis for social objects through grounding in consensual reality (defined later in this chapter) (Moloney, Hall, \& Walker, 2005; D. Rose et al., 1995).

Common sense provides mutual ground for people to discuss, argue, and negotiate. Consensual reality does not imply that individuals hold the same views, rather that there is an awareness and understanding of the common views by all individuals, such that even though an individual may not personally agree with one or the other views, they can easily engage in discussion involving one or other viewpoints D. Rose et al., 1995 in Moloney et al., pg. 417 .

From this perspective, common sense is mostly demonstrably factual information, but that is (at some level) removed from micro-scientific explanations (Bangerter, 1995). An important point to appreciate is that common sense knowledge can be approached from multiple referential perspectives, yet still be understood widely. For example the statement "Rain is Wet" is common sense knowledge. Whereas a purely factual logical reductionist might expand this piece of information into something more akin to "Rain is water, water is a liquid, liquids have certain properties Including fluidity, Fluidity is perceived as wetness by human senses" (Fluid - Wikipedia, the free encyclopedia, n.d.). This could be further deconstructed into its principle scientific presuppositions each of which can be verified as inductively true (with the notable exception of human perception). However if you are not a Chemist, Physicist or other specialist interested in communicating with your own community of practise about these properties, it is makes sense to accept that rain is indeed wet for the large majority of communicative and cognitive events in an individuals life. More importantly making the inductive leap that other individuals also share this cognitive construct of knowledge about a phenomenon is what differentiates between individual belief and common sense.

Breaking this simple statement down further from a socio-linguistic semiotics (Levin-Rozalis, 2007) perspective offers an alternative to a purely scientific-reductionist perspective, and is somewhat more akin to the way 
in which common sense is used within Social Representations literature. Within this tradition, rain is the signifier for the signified - water that falls from the sky. Further, this statement provides a concrete example of a property of the signified which is being wet. This type of linguisticreductionist perspective is used widely within discourse analysis (Potter \& Edwards, 1999; Billig, 1996; Potter \& Wetherell, 1987), which itself draws heavily on methods established in the tradition of conversation analysis (Sacks, Schegloff, \& Jefferson, 1974). Indeed many scholars have argued for social representations to be used in conjunction with techniques such as discourse analysis (Antaki et al., 2003; Potter \& Billig, 1992; Potter \& Edwards, 1999) for the principle method of establishing social representations from a corpus. Metaphor arguably is the most important bridge between the two theoretical backgrounds. Metaphor within social representations theory is key to the ideas of anchoring and objectification, the processes whereby concepts and information are mapped to a pre-existing social object, inheriting the properties associated with the original object in a process analogous to the mapping and domain processes inherent in Lakoff and Johnson (1980c) metaphor studies. While this is still a somewhat cognitive, individual sense-making perspective, the ways in which common sense are construed through metaphor is arguably a discursive process. Both this and the Scientific-reductionist perspectives both make the reduction of common sense statements the paramount goal in describing how understanding and shared meaning are achieved. The shrewdness which comprises both of these approaches will be discussed later. The important point being made for the moment is that understanding common sense is based on analysis of component parts of communicative acts. And with the basis of these component parts at some point drawing from a referent source. Thus in the example given above, the referent source of the knowledge is, or has become, science and scientific processes - at least for instances of common sense which draw upon physical observations and phenomenon. A purely linguistic model becomes more useful when dealing with common sense about topics which are not physical/factual in nature.

Bangerter, 1995 uses an example taken from Rastau (1998) illustrating 
how religious knowledge can also be split into a similar dichotomy. In the example given, Bangerter describes how Rustau observed the transmission and maintenance of Brahman texts within the Hindu religion throughout time. The texts are venerated and within the religious community of practise are understood to be referent, such that meaning and symbolism can only be interpreted in a specific fashion. The maintenance of these referential interpretations is produced through a complex hierarchy of rote learning, specific phonetics and a specialised dialect. Implicit in this is the understanding that the everyday use of passages by the non-elite members of the religion would lead to misinterpretation or impure or incomplete recitations. This Sacred and Profane dichotomy as Moscovici calls it, drawing upon the Romanian religious-psychological theorist Eliade (1961) work on religious symbolism. This dichot implying that the sacred contains all information in a pure form, and the profane that which is used and exists in a corrupted state. This distinction exists in many societies and domains of knowledge, but at its core merely marks a distinction between common sense and expert domains of referent knowledge.

The utility of invoking this dichotomy is obvious in that it represents the communicative distances between groups in a society. Both common sense knowledge and referent knowledge are closely intertwined with the community of practise and cultures in which they exist (Bangerter, 1995). Referent sources, regardless of the domain or culture, are tasked with the maintenance and production of knowledge, and are only given referential status by the society in which they exist. For most contemporary secular societies, this referent domain is science. However, as Moscovici (Moscovici, 1984) points out, they are also tasked with propagating and integrating with common sense knowledge. Thus, attempting to bring the representation to be something which is more referentially aligned with the originating groups social object, yet which is still accepted and accessible by the masses. It is this process which social representations theory seeks to, in part, espouse. 


\subsubsection{A Legal Example: Human Rights Violations}

While discussing common sense in terms of scientific and religious domains as above, a more protracted and relevant example is to observe the concept of common sense using a legal referential domain. The purpose of this is to demonstrate the applicability of the SR concept of common sense to a context that much of the subject matter of this thesis will involve. The idea of political and legal referential authority stemming from a society's sense of what is good and bad is a topic which is addressed at length in a number of chapters from the excellent reference work the Psychology of Rights and Duties (Finkel \& Moghaddam, 2005), and in particular to the chapter by Spini and Doise considering 'Universal rights and duties as normative social representations'. Within the chapter the authors categorise a number of domains that are drawn on to represent rights and duties within a society. They note that within a framework of rights and duties the expert 'frames' draw from primarily legal, historical and political points of view (Spini \& Doise, 2005; Doise, 2001; Doise, Spini, Jesuino, $\& \mathrm{Ng}, 1994)$. While targeting representations of rights and duties, the authors refer to their previous studies examining the common sense views (Doise et al., 1994) about human rights held by 'laypersons', and other research covering over a decade of collaborative work within primarily European and Western cultures. In the original 1994 study they use a qualitative framework with instruments derived from the Universal Declaration of Human Rights (Universal Declaration of Human Rights - Wikipedia, the free encyclopedia, n.d.) as a basis for an expert political/legal referential domain. The object of the study was to elicit common sense conceptions held by various groups about what constituted violations of human rights, therefore implicitly identifying the common sense representations of human rights through the observance of the boundaries of what was considered a violation, and what was not. By using a survey style questionnaire designed to elicit responses to items which portrayed violations of various rights as outlined in the original declaration, the researchers hoped to infer common sense representations of human rights within the five countries sampled. The five countries sampled were all constitutionally aligned with the statements outlined within the universal declaration 
of human rights as committed by the UN in 1948 (Universal Declaration of Human Rights - Wikipedia, the free encyclopedia, n.d.), and had faced significant political restructuring after World War 2. This study showed very little variation between countries, implying a shared/common sense understanding of human rights (Clémence, Doise, Rosa, \& Gonzalez, 1995). More recently (Clémence, Devos, \& Doise, 2001) has extended the original research using similar methodologies. The topics of human rights and human rights violations are a well investigated topic by social science researchers outside of the social representations field. See (Sidanius \& Pratto, 1999) for Human rights violations in regard to Social Dominance Theory and Right Wing Authoritarianism approaches, Huguet, Latan, and Bourgeois (1998) and Cohrs, Maes, Moschner, and Kielmann (2007) for an integrative review with a emphasis on attitude and behaviour perspectives.

Spini and Doise (2005) present a review of various studies concerned with representations of human rights, including a further cross-cultural study employing similar methodologies to those adopted by Clémence et al. study. In this study (Clémence et al., 2001) 16 countries were selected based on a variety of cultural differences (Hofstede, 2001) and demonstrated wide-ranging evidence in support of three distinct clusters of human rights. Using a cluster analysis (Spini \& Doise, 2005) based on the cross-cultural study (Clémence et al., 2001) the following clusters were observed: Basic rights, socio-economic rights, and rights and duties of the individuals. Little difference between countries' common sense representations of human rights were observed. Where variations appeared they were said to account for what is described as 'extended definitions' of human rights violations. These variations are anchored to (Clémence et al., 1995) social belief structures, and also to demographic factors such as age and socioeconomic position. In particular, older participants had greater extended (more referentially aligned to the Universal Declaration statements) definitions of Human rights violations, and included less atypical definitions, a process which the authors attribute to increased socialisation (Clémence et al., 1995; Diaz-Veizades, Widaman, Little, \& Gibbs, 1995).

Demographic factors are of particular relevance as alternative explana- 
tions rather than relying on increased socialisation explanation for the increased normality of common sense representations in older populations. The effect could quite simply be increased exposure to referent source material in certain demographics- notably from generations with less generational gap between major world events instrumental in the legal legitimisation of human rights discourses (i.e. World Wars). The importance of these studies is that they examine 'common sense' in detail, illustrating that there is much homogenisation apparent across widely divergent groups separated geographically, politically and culturally. These studies highlight the importance of institutions, and in particular the role that world events play in the formation and maintenance of collective histories used to anchor current common sense positions (J. H. Liu et al., 2005). Common sense, when positioned within SRT, represents a more homogeneous macro-level understanding of concepts than social objects(discussed in the next section) or social representations do as a whole, relating widely held understanding across group boundaries. The politically and geographically distinct groups in the studies cited showing divergence only in common sense human rights understandings of what could be described as culture or individual level bound attitudes (Clémence et al., 1995). These were rights where individuals had a higher or lower degree of control (rights of children, rights of spouse, rights of movement) dependant on the societies they lived within.

\subsubsection{Social Objects}

For Social Representations Theory the idea of social objects stems from how an object exists in the social interactions of a group or society, the idea that groups and constituent members base behaviours, beliefs and values around these structures being central to the theory of SRT. SRT suggests that for a concept to be a social object it must exist first as part of a common sense understanding (Wagner, 1998; Poeschl, 2004). Wagner (Wagner, 1998) and Moscovici (Moscovici, 1988, 1984; Moscovici \& Markova, 1998) go further to suggest that for any observable human interaction there must exist a social object, beyond that of "brute facts" as evidenced in the previous section about Common sense knowledge. SRT suggests that only after 
attributes are ascribed to objects can they be navigated. Even when applied to scientific knowledge, objects must become social before they can be used in communicative exchanges. To use the example from Bangerter (1995) which discusses how even fundamental scientific objects such as quarks (the basic sub atomic unit) lack any properties until their relationship to other previously existing atomic elements such as electrons, atoms, is articulated either linguistically or mathematically. This demonstrates that even referent (sacred) knowledge itself exists within a world of social representations. This observation of the theory had been somewhat murky, and attempts (Bangerter, 1995; R. M. Farr, 1993) during the early nineties to address the problematic issue suggest that there may never be a truly definitive reference domain of knowledge. The classic dichot of science vs common sense becomes pivotal to the development of discourses leading to the clarification that there exist varying levels of representation, and further, that they are not always contradictory. Indeed when viewed within the social objects framing, which applies to all communicable properties, knowledge dichotomies become redundant (Bangerter, 1995)

Within a polychotomous world, objective positions are difficult to maintain, and so social objects bridge this purely positivist conception of reality to one where groups are able to construct stable representations, particular and referent, to their own group(s). Group members become responsible for constructing, maintaining, and influencing the social objects and associated beliefs and attitudes out of pre-existing social objects available to them. This determines the properties that are maintained or discarded, largely independent of the perceived legitimacy by outsiders. The stability of social objects underpins Moscovici's statement that representations are resistant to change. However, this property been questioned recently (Poeschl, 2004) because individuals' positions within a social structure may change, and in turn lead to representational variations, leading to the proposition by Poeschl (2004) that the plasticity of social thinking may undermine this notable resistance to change found in social representations, through the mechanism of context sensitive social objects. 


\subsection{Institutions and Groups}

The role that organisations and institutions play in terms of attitudinal change and behavioural models and norms perhaps make them the largest transmitters of both referent knowledge (Baerveldt \& Voestermans, 2005; Verheggen \& Baerveldt, 2007) and legitimising myths (J. H. Liu et al., 2005; Verkuyten \& Brug, 2004; Sidanius \& Pratto, 1999) within and across societies. The role that corporations, NGOs'(non-government organisations) and, more traditionally, the State, have over how objects are disseminated and endorsed within societies provides a platform for the anchors of social representations, through the collective framework of symbols (J. H. Liu et al., 2005) such as flags, mottoes, and public holidays. Institutions also provide fora for discussion about new concepts, information and events, and through this process influence the environment in which groups operationalise them. The power of institutions to maintain existing social representations through a status quo is perhaps their most important contribution, discussion of which draws parallels with Social Dominance Theory (Sidanius \& Pratto, 1999). Religion, for example, is given legitimacy through a state's acknowledgement of religious holidays through instituting in law public holidays. Likewise, trade and treaty agreements are maintained and legitimised by the influence they have on overriding public opinion and discussion of various legal rules present in a society. In the private sector, product advertisements and placements can produce a set of familiar symbols which are used in turn by a society to represent certain qualities or characteristics, and by an institutions maintenance of the symbol in the public eye a status quo over the links between certain symbols and qualities; Coca-Cola, Mickey Mouse, McDonald's, for example. Going beyond just corporate marketing these are products or symbols that are maintained by institutional interests as salient icons, as much as they have come to be maintained by societies themselves as they have become social objects in their own right. 


\subsection{Themata}

The role of themata within social representation as discussed in Markova (2000, 1996); Moscovici and Vignaux (n.d.) is presented as being embedded within the dialogical perspective of SRT. Because SRT carries with it a tradition of semiotics, arising from the European cultural-semiotic philosophy and linguistics schools of thought, themata, taxonomies and thema are used in ways indicative of oppositional dichotomies that underlie common sense narratives (Markova, 2000). Themata can be conceptualised as dichotomies or polychotomies, almost invariably opposed, or situated on a continuum of opposition (Moloney et al., 2005) such as dirty/clean. Being considered the underpinning of common sense knowledge, they are rarely brought into question or reflexively examined in spontaneous discourse. In many ways, themata are to common sense, as common sense is to a social representation, meaning they present the smallest divisible unit of the greater theory. In (L. Liu, 2006) the discussion of quality of life in China is framed as a conceptual exploration of themata in social representations. The author L. Liu posits that quality of life is linked to themata which are inherently less developed in Chinese society than the counterpart representation in the west. The author cites possible changes in economic imperatives and ideals transmitted through the politico-legal institution, that differ from those in the that were instilled during the cultural revolution (L. Liu, 2006). The underlying properties to which quality of life draws upon being; personal assets, purchasing power, living conditions and market availability - these are inherently capitalist and individualistic societal ideals anchored to the basic themata of having and being. By using these themata in examining the quality of life concept in China, L. Liu (2006) is able to observe divergences from developed individualist nations social representations of quality of life, and how this is intertwined in the common sense understanding of the same. L. Liu (2006) demonstrates that the same themata are present in a growing middle class of Chinese society. At the same time, for a large proportion of rural Chinese society, quality of life is anchored in more collectivist and communistic ideals centred around traditional family and community roles - towards the being continuum. 
Because of the relatively recent introduction of themata to the general dialogue surrounding social representations theory (Markova, 2000; Markov, 2003) the position and utility has been a recent point of discussion (Voelklein \& Howarth, 2005). Themata fit naturally within the process model somewhere between discourse within society, and initial anchoring and objectification by groups, perhaps in what is best described as a navigation phase. When observed as domain-free metaphors - ones lacking any concrete mapping (i.e. unlike the instance of a pipes metaphor for communication, where pipes provide a concrete physical basis for the metaphor) (L. Liu, 2004) they demonstrate an interdependence between common sense thinking and social morphology. This could tentatively come under the banner of a component of sharedness (Moloney et al., 2005).

\subsubsection{Taxonomies}

Taxonomies can be generally considered as a more diversified form of themata. While themata tend to occur as dichotomous pairs, commonly opposed - good, bad; having, being; goals, needs - taxonomies are collated lists of commonalities, such as commonly occurring and conceptually linked themes. They are clustered along with thema on what can be considered a representational field along a continuum of opposition (Moloney et al., 2005). Thus, they can be considered organised or grouped terms that commonly appear around a particular set of thema at towards either end of an oppositional continuum.

To this end, simplistically Themata can be considered a macrog or generalised form of a taxonomie, whereas taxonomies constitute lists of attributes belonging to a more generalised form, a themata.

\subsubsection{Contradiction Accommodation}

Social representations are defined when there is social and technological change (Wagner, Kronberger, \& Seifert, 2002), during times when conflicting ideologies as well as new knowledge are being introduced to the common sense world (Moscovici, 1988). One of the final processes occurring 
during generation of a social object (Wagner et al., 1999; Markova, 2000) as discussed in the next section, is contradiction accommodation. This is the notion that incorporating conflicting elements of a new social object into the encompassing social representation must happen via social identitylike processes. Describing groups that are able to incorporate elements which may oppose their own conception of a social object. To borrow from a study by Wagner et al. (2002) investigating Social Representations of biotechnology in Europe over a period from 1996 - 1999, contradictions are incorporated in such a way as to retain local consistency (McGuire, 1986) and so as not to cause dissonance within a given representation. In Wagner et al. (2002) genetically modified tomatoes are seen as both good; increased yields, disease and pest resistance, higher economic yield - and bad; against religious values, potential harmful cross-pollination, genepool homogenisation, non-renewable crops, poisonous crops, etc. Contradiction in terms of themata is generally placed within good/bad pairs, and their appearance or absence in group positions is a powerful factor in social identity formation (Elejabarrieta, 1994).

\subsection{Process model or Sociogenesis of Social Rep- resentations}

Figure 1.1 on page 23 presents a graphical depiction of social representations or what can be called the process model. First proposed by Wagner et al. (1999) in a concise overview of the Theory of Social representations (Wagner et al., 1999). As a tool in understanding the cyclic nature of social representations and in particular the relationships to collective coping, the cyclic model is useful for visualising the various events occurring during the generation and adoption of a SR. With an emphasis on the role of social objects and anchoring and objectification processes. It presents both a macro-level perspective of the social processes at work, and offers a coherent way of conceiving of the component elements.

The model represents what has been alluded to as Wagner's epistemology of social representations (Voelklein \& Howarth, 2005) and as such in its original 1999 presentation lacks some of the elements discussed here. 


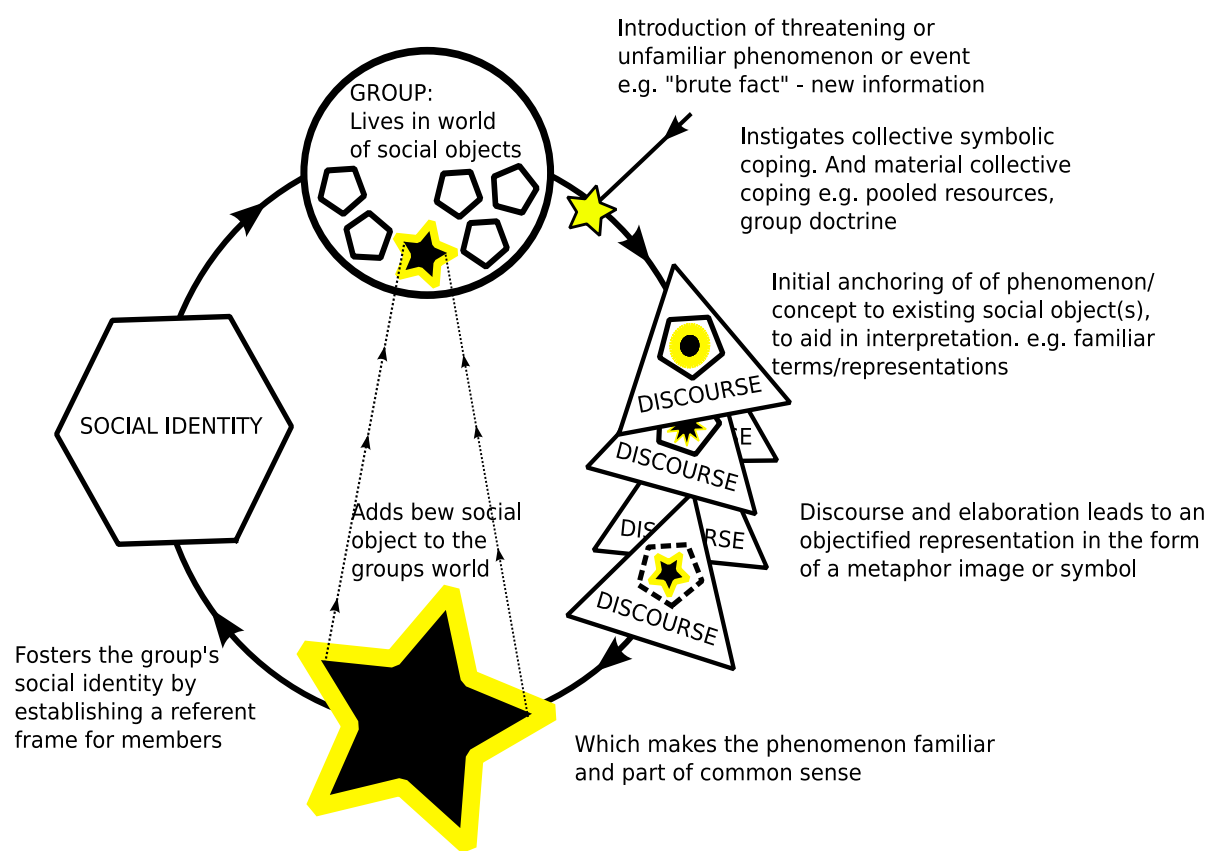

NEW SOCIAL REPRESENTATION

Figure 1.1: The Sociogenesis of Social representations process model adapted from Wagner et al., 1999 which incorporates most widely used elements of social representations 
In particular a discussion of Markova and Moscovici and others presentation (Markova, 2000; Moscovici \& Markova, 1998) of themata and only implicitly draws on the dichotomy/oppositional concepts of referent and common sense knowledge. The process model places a heavy reliance on; social objects, anchoring and objectification, and group integration of these - sometimes referred to as core elements of SRT. It's strong points lie in providing insight into how social representations influence, and are in turn influenced by, social identity, and the associated theory (SIT) (Tajfel \& Turner, 1986). It also draws heavily on the concept of social objects, discussed previously in this chapter that, within the sociogenesis model, are viewed as the foundation of social representations. The process model itself forms an iterative, recursive system, where social groups manifest, create and orientate around worlds of social objects. The cyclic nature is a core concept which can be lost when dredging through the various literature on the topic. The recursive nature of identity formation, informing and being informed by the creation of new social objects by the anchoring of new phenomenon to familiar concepts found in pre-existing social objects, and the subsequent objectification into the new. These are undoubtedly the most widely utilised aspects of Social Representations theory within the Social Sciences (Pawlowski \& Nenov, 2004; J. H. Liu \& Sibley, 2006; Wagner et al., 2002; Markova, 2000; Byford, 2002; Herrera, Lavallée, \& Doise, 1998; Potter \& Billig, 1992, 1992; Räty \& Snellman, 1992).

The role of conflict is also incorporated within the model, both in the introduction of threatening or unfamiliar new phenomenon or concepts in a groups social world, via brute facts and, secondly, in the discourse that occurs during this symbolic coping. This conflictual process in turn leads to a social representation incorporating the new social object, through the anchoring and objectification mechanisms. This is then legitimised through group identity formation, and maintenance of a particular social representation occurs via the legitimised group referential integrity, leading to the introduction of new and/or modified social objects to a group's repertoire. Individuals' own sense making of new phenomenon is determined by how much they ascribe and align to particular groups, via individuallevel social identity processes. Let us now examine in more details the 
components within the sociogenesis model further.

\subsubsection{Brute Facts}

Conflict, as a theme within social representations, is facilitated primarily by the introduction of brute facts into the world of the social object which cannot be incorporated within an existing representation. Defining the concept of brute facts late into this overview of various components within social representations theory provides a deliberate separation from the common sense/reified world dichotomy rhetoric. Brute facts may essentially be defined as discrete units of knowledge that at some point continues to just be regardless of referent, reified domain, or the group they emanate from. As already illustrated, a referent domain and its preexisting world of social objects must exist for any piece of information to be observably utilised. This does not, however, discount its existence as a purely social constructivist approach might imply. A classic example might be the tree that falls in the woods with no one to observe it - does it still make a noise? Inductive logic would tell us quite clearly it does. Conversely the argument for the existence of a divine entity, by way of a purely social constructivist viewpoint, clearly does exist. This might be demonstrated by the real, observable, effect on people's behaviour and communication. To borrow from discourse analysis (Antaki et al., 2003; Wetherell, 1998), the concept of God is demonstrably 'done'. Very clearly there exists a Social Representation of the same concept. However the concept of God, unlike the sound of surface friction and object mass displacing the surrounding air, is not a brute fact. For Wagner (1998) for a brute fact to exist it must meet a number of criteria; principally that it challenges any number of given pre-existing social representations and associated social objects, forcing dialogue, and in turn influencing social identity. To return to the sound of objects falling, regardless of referent social group (this event implicitly involves a number of conditional criteria, such as surface friction, mass, atmospheric pressure, velocity, a number of which are not immediately observable to a human spectator in any case) there is a brute fact that in many cases falling stuff makes sound and/or vibrations. For the case of the existence of God, there would need to be some 'brute 
fact' which would challenge, not just select social referent groups existing consensual representations about social objects, but force reconsideration among most (if not all) social groups. The topic of religion is a useful one to demonstrate the separation of brute facts from any given referent social group, in that it is integral in most religions to share a fundamental set of representations of social objects of the world in which members exist that run counter to other-group conceptions of the same social objects. In this example, the Evolution/Creationism debate might be one of the most salient.

Technology, Biological Processes, and political unrest are all favoured topics for Social representation research (Gaskell \& Bauer, 2001; Moscovici \& Markova, 1998; Wagner et al., 2002; Wagner \& Hayes, 2005) for the reason that they all introduce 'brute facts' into the social worlds of society. For topics such as Biotechnology (Gaskell \& Bauer, 2001; Wagner et al., 2002; Herder \& DyckBrian, 2008), brute facts come in many forms; economic impacts, challenges to views about natural order, challenges to religious doctrine. Normally in the realm of technology brute facts often occupy a single or limited reified domain long before they become a topic of common or consensual discourse. As in the case of genetically modified food crops, however the transition of this knowledge into a brute fact comes generally when two reified domains collide bringing the brute fact to salience outside of it's original domain, and triggering the process of collective symbolic coping.

\subsubsection{Collective Symbolic Coping}

Collective Symbolic Coping is a term used to describe the process that societal groups go through when new information is introduced into them, often in (but not limited to) the form of brute facts. There are four steps to collective symbolic coping as proposed by Wagner et al. (2002).

1. the creation of awareness

2. production of divergent images

3. convergence upon a couple of dominant images in the public sphere 
4. normalisation

Generally collective symbolic coping occurs when significant media attention, policy discussion, activism or economic impact, are related or attributed to a given topic or concept that cannot be incorporated in a social representation easily using existing social objects. Generally, representations formed as a result of the process do not rely on pre-existing knowledge (using the biotechnology example this would be pre-existing scientific knowledge). The new representation forms by coalescing and anchoring to particularly salient media events, images or icons. Thus, in instances where collective symbolic coping is identified as occurring, media institutions are known to have greater impact upon societal representations of the topic (Wagner et al., 2002) by providing a dominant image/discourse for initial stages of SR formation. Highly salient images/discourses are made available across social groups and provide the basis for consensually agreeable components of a concept to emerge.

\subsubsection{Consensus}

Consensus between social groups forms late in the sociogenesis process of social representations. While consensually identifiable images may be guided by institutions and media early on, having clearly identified group positions that are consensually identifiable and belonging to a particular group marks the end of the anchoring and objectification process. While anchoring and objectification warrants its own section, it is sufficient to briefly say that it is the process of using existing social objects, first in an attempt to map characteristics and then during objectification, to ascribe new meanings to these mapped characteristics. A full discussion of this process follows later in the chapter. Consensus solidifies the shared meaning of an objectified object that might otherwise disappear or change. When a particular social group is able to effectively agree upon properties of social objects, it maintains the social representation, not only of an objectified object but also potentially the renegotiation of existing social objects. Since SRT does not preclude variation of individuals and the shifting of a social representation inherent through time (Bauer \& Gaskell, 
1999), consensus can be observed primarily by the subsidence of discussion around thematic sets of social objects (D. Rose et al., 1995). Consensus in many cases is marked by public statements or some form of domain referent liturgy being issued. In the case of Social representations of Genetically Modified Organisms (GMOs) in the EU (Wagner et al., 2002), the authors make reference to a relatively late Media release from the Greek Orthodox Church in 1999, speaking out against GMOs. While they had observed high incidence of societal discourse as measured by media and public debate prior to the church's announcement in 1999, the Church's statement represents what might be considered a good indicator of consensus following the most intense period of discourse. While institutions play an important role in helping form Social Representations, this particular example illustrates a referent group forming consensus because of the highly non-secular nature of Greek society and the embedded-ness of the Greek orthodox church in daily life for a large section of Greek society. The position of the church represents a common sense consensual position as determined by its influential position in the social identity of groups in Greek society. This example also demonstrates how different groups can take social objects and subvert the original referent domain transmitters social object - the church in this case assumes a referent consensual position by virtue of majority in-group referent position through religious doctrine. The consensus provided through doctrine and edicts provides support to the maintenance of a stable social representation (Wagner et al., 1999) and enables the addition of a new social object to the group's world so the social identity orientation and formation processes can begin.

\subsubsection{Social Identity}

The way social groups inform and influence identity is, if not completely understood (J. H. Liu, Wilson, McClure, \& Higgins, 1999; Robinson, 1996; Yamagishi, Mifune, Liu, \& Pauling, in press), fairly established within social psychology as a whole. Much of this understanding stems from the seminal work of Tajfel and Turner (1986) on Social Identity Theory (SIT), written in response partly to questions around inter-group conflict. SIT has evolved over time, and has formed a significant part of social represen- 
tations theory (Moscovici, 1988) since SIT gained momentum within social psychology. For SRT it is the mechanism for how social groups, and the representations they harbour, influence the behaviours and attitudes of individuals. SIT brings a loose collection of related theories and themes under one banner . Social representations, as discussed in Moscovici (1984), have since used SIT as the primary mechanism by which attitudes and behaviours are assumed to work, as well as the final component in the formation and maintenance of social objects in the world (Howarth, 2002). The four principle mechanisms of SIT are:

1. Categorisation - putting self and others into a category to produce meaning by associated labels, e.g., They are German, I am a Liberal.

2. Identification: Association with our in-groups, to bolster self esteem.

3. Comparison: We compare our groups with other groups, placing favourable emphasis on our own.

4. Distinctiveness: We desire distinctiveness and positive comparison with other out-groups

While these four tenets have come under scrutiny, with a shift in emphasis from in-group favouritism towards a model of perceived reciprocity being proposed as being the fundamental factor in determining behaviours (Yamagishi et al., in press; Yamagishi \& Kiyonari, 2000). They remain useful for supporting SRT in providing explanations or predictions for how inter-group interactions may occur. In terms of completing the sociogenesis process, SIT is simply how a given social object may influence the orientation for, or against, other groups holding alternative social representations. For example, a group that forms a negative social representation of genetic engineering may resent a group which forms a favourable social representation of the same. The influence of the anchoring and objectification process becoming apparent in the attributes groups may emphasise in their given referent social object. To better understand how different attributes and ultimately how these in turn form biases based on group membership we need to review in detail the anchoring and objectification process. This is addressed in the next section. 


\subsection{Anchoring and Objectification}

Social representations are themselves built upon pre-existing representations in much the same way as interpretive repertoires are used within discourse analytic frameworks (Augoustinos, LeCouteur, \& Soyland, 2002; Billig, 1996; Potter \& Wetherell, 1987). In turn this creates a self-referent recursive structure within a given social representation, in that pre-existing social representations are used to anchor newly introduced topics, and this in turn changes those existing social representations. Continual anchoring and objectification is arguably a product of modern societies, where new information is rapidly and continuously introduced to many disparate groups. These intra-group communications are facilitated through the use of shared points of reference, or collective representation.

Objectification is a continuation of this generative social representation process, while it is not always present for any given social representation it describes the process whereby an anchored representation comes to embody the living truth regardless of factual content. Objectification is purely constructivist in nature, whereby discourse becomes reality by providing its own version of an underlying objective truth (Byford, 2002). Understanding when, and if, a given social representation has come to be objectified demonstrates a pervasiveness of a given underlying discourse or ideology over the continued evolution of any given social representation.

The relationship between Anchoring and Objectivication is both in terms of a timeline of the process (anchoring must occur before objectivication), and in terms of underlying informing theory. Anchoring being somewhat akin to metaphor production, and thus bounded by linguistically grounded theory. Whereas Objectivication is the contextualisation of the initial anchoring based on new information that becomes avaialable, and is more grounded by social and group interactions process and theory.

One of the most important aspects of the anchoring and objectification process is the initial anchoring to existing social objects. This occurs normally in response to a lack of information in existing social objects groups have at their disposal as explanatory structures. The anchoring process is most structurally similar to metaphor concepts hinted at earlier in the chapter, and in metaphor terms is a direct mapping of a referent domain 


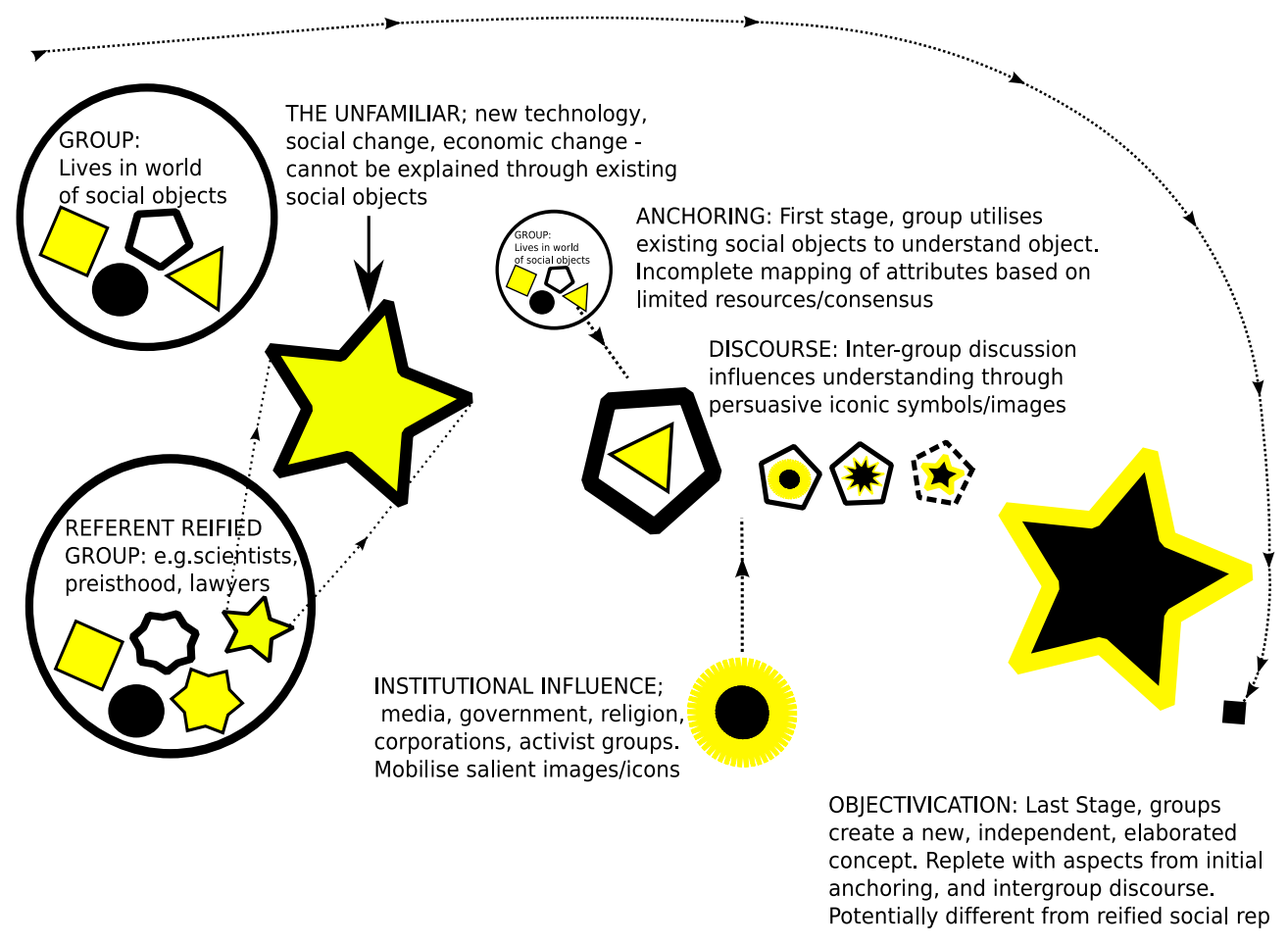

Figure 1.2: The Anchoring and Objectification process in detail

system onto a target domain. As such, examining how social representations are initially anchored using a metaphorical analysis approach can shed a great deal on the end objectified structure. Of course the main purpose is to enable navigation of unfamiliar concepts and information. To make the unfamiliar, familiar.

\subsubsection{Making the unfamiliar, familiar}

SRT is commonly framed by its functional qualities as a theory. One of these functional qualities concerns how an SRT allows new concepts to be made accessible. When a new technology is introduced it can have the potential to disrupt and significantly impact upon established norms, values and behaviours (Wagner et al., 2002). The processes by which a new technology achieves these impacts are often beyond comprehension of all but a few groups. Thus, social representations are formed to allow affected groups the ability to cope with a new phenomenon. To do this 
they must take something apparently arcane, and form meaning around their interactions, direct or not, with it.

Sometimes technical/expert referent knowledge associated with such technology is assimilated or transferred into a usable form through various institutions to be used by the general public. The resultant social representation is then used as an anchor for subsequent interpretation of new innovations. Depending on the type of Technical/Expert Knowledge involved, the societal-level SR may diverge significantly from the original meaning and purpose of the often originally reified knowledge. Räty and Snellman (1992) utilise this concept in their notes on Criticisms of SRT, with the following quote from the introduction attempting to summarise SR within the familiar/unfamiliar framing. This is a classical SRT view of the function of social representations (Moscovici \& Markova, 1998).

Social representations are characteristically a phenomenon of a modern society, a society in which changes are too rapid to precipitate into permanent traditions. Their essential feature is the effect of science, a reified universe, on everyday consciousness. Social representations have their origins in the abstract, technical concepts of science, which are transformed into comprehensible, familiar and safe ones in the consensual universe.Räty and Snellman (1992, pp 4)

This quote succinctly summarises classical SRT, and the emphasis can clearly be seen in the making the unfamiliar familiar theme. The reason I have chosen to place it within the anchoring and objectification subsection rather than at the beginning of this chapter is because SRT, as it exists today, is far more wide-ranging in its scope. Anchoring and objectification is still very much at its heart in terms of its functional components, rather than its more philosophical bent aspects, and deserves to stand out more fully than other aspects. As a rhetorical and interpretative tool, it stands to be the most fully operationalised in the rest of this thesis, using a discourse/conversational semiotic linguistic tradition of metaphor analysis to bridge a linguistic/discourse tradition with SRT. 


\subsection{Metaphors and Mechanisms}

The role metaphors have in fostering understanding of concepts is an important and well established one. The use of Metaphor as a tool for analysing understanding is employed throughout Language Science and Social sciences, and as far ranging as Law and Computer Science (Lakoff \& Johnson, 1980c; M. Rose, 2002a; Barr, 2003; Lakoff \& Johnson, 1980a; Gannon, 2001; Yu, 1998; Potter \& Wetherell, 1987; Antaki et al., 2003; M. Rose, 2003; Billig, 1996). The key role and feature of metaphor is to map from one domain onto another, transferring features of the original referent domain onto the target. Among a number of classic examples one of the more pervasive is the Pipe domain, where pipes are used as a reference domain. The reasons for the pipe metaphor's prevalence are manifold, the properties which are most commonly mapped are the physical ability to conduct the flow of their contents, to connect to other pipes, to terminate, leak, and extend from a reservoir. Not only this, but they are a common object encountered in everyday life. These features are used widely, from computer metaphors to knowledge, electrical circuits, communication, and performance, among other target domains. It is important to point out the use of the term 'domain' when discussing metaphor revolves around properties, generally physical but not necessarily, rather than a specialised SRT conception. The classic studies by Lakoff and Johnson (1980b) serve as a basis for a number of wide ranging interpretations of how metaphor can be used within more general theory of mind and society. Indeed metaphors are honoured within social representations theory as a pivotal component of the anchoring and objectification process. For without metaphor, or as it is termed more definitively within social representations theory anchoring, there is no process of social representations - only a loosely bundled set of dialogues. Themata also share most if not all the features of metaphor, and could at least in some instances be considered as such. For example the thema of good and evil can be expressed as a metaphor of evil (such and such is evil) within a metaphor construct if something is labbeled evil there is a highlighted aspect of bad/detrimental, (which is in contrast to a hidden contruct of good/not evil). Metaphors are useful to people because of there ability to highlight aspects of concepts and systems which 
might not otherwise be immediately obvious, and they also tend to simplify concepts so that a greater range of people can understand them. The converse of metaphor is that they can hide certain features, and also introduce inconsistent or irrelevant features which may not exist in the target domain. One example might be the use of a Lego block metaphor for molecules. While the Lego block metaphor adequately demonstrates the positive and negative forces involved, and also the joining of different substances, it cannot map such concepts as electron shell around an atom, the strong and weak forces involved in molecular bonding, or the exchange of elementary particles between molecules to form isotopes. Highlighting and Hiding (Lakoff \& Johnson, 1980b) are important to consider when examining anchoring and objectification processes, because of the qualities and degradation or supplanting that occur during this stage having wide ranging influence.

\subsection{Summary}

While social representations theory may appear to present a somewhat fragmentary guiding theory, it also offers a flexible frame for an exploratory thesis such as this one. Because of its fundamentally qualitative reflexive approach to itself, it is sometimes difficult to understand how one researcher to the next has employed and operationalised a particular term or presented a piece of research. However, in its ability to observe how abstract concepts permeate into a society as shown in fields of; technology, human rights, medical understanding among others - it is perfect for the topic of this thesis. There is no other theory which has been applied to such wide-ranging or abstracted topics within social sciences, or addresses the societal conceptions in the same way. 


\section{Chapter 2}

\section{Methodologies}

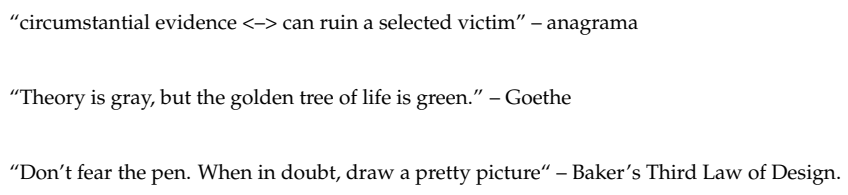

The principle theory being employed throughout this work is Social Representations Theory (Clémence et al., 2001; Moscovici \& Markova, 1998; Wagner et al., 1999) which does not by itself provide a particular methodology. Social representations theory is covered in depth in the Chapter Social Representations starting on page 7. A brief recap of Social Representations is that it is a theory of observing socially constructed objects of understanding and how understanding of those objects comes to be achieved, maintained, and altered by groups encountering them. It is focused primarily at a societal level with the presumption that codifying social groups' representations of the world and objects within that world allows a better understanding about how those groups might interact on a societal level. How social representations are accessed by a researcher investigating any given topic is controversial within the literature (Voelklein \& Howarth, 2005; Doise, 1993) and left somewhat open to investigator discretion. I have chosen to employ thematic analysis as the analytical methodology, and to use Social Representations as the principle theoretical framework which to draw observations derived from thematic analysis (Braun \& Clarke, 2006) into.

The research questions I hope to answer are: 
- To identify if there are social representations of a globalised concept of abstract knowledge and if so how do different groups realise it?

- To identify elements of the social representations and associated themes, present in modern day social objects as well as attempt to identify historical representations of ICW ${ }^{12}$

- Identify groups which claim to have stake in ICW, and if present, the features of conflicting and contradictory representations, and how and why contradictory representations are held.

The reason for undertaking this is grounded in the current controversies surrounding copyrights and patents under globalisation that have been made apparent by recent changes in business practises, electronic media and communications technologies, and the impact they have on societies and individuals' lives. Many researchers (Vaidhyanathan, 2001; Benkler, 2004; Lessig, 2001; Hesse, 2002; Ruttan, 2006) in other disciplines ranging from Law to Economics and History have attempted to put current debate surrounding these changes and challenges into perspective from there respective disciplines. I hope to add a social psychological perspective to these and other researchers' efforts into the general area of intangibles and information resource practises within societies, by illustrating the representational practices of various groups engaged with the topic.

\subsection{Thematic Analysis}

Thematic Analysis (Braun \& Clarke, 2006; Aronson, 1994; Boyatzis, 1998) provides a structured framework for doing qualitative analysis, while still

\footnotetext{
${ }^{1}$ I operationalise this abstract knowledge/ideas expressions and thought as Intellectual and Creative Work (ICW) throughout the thesis, the work suffix included in the phrase is a little misleading, but it is included with intellectual and creative categories to qualify the concept with some sort of input/output

${ }^{2}$ Richard Stallman a Software developer and rights activist provides an overview of why intellectual property is a loaded term in the article Did You Say "Intellectual Property"? It's a Seductive Mirage" (Stallman, 2004)
} 
retaining the reflexive flexibility required for analysis of abstract and fundamentally socially constructed object of investigation. Unlike similar methodologies such as content analysis the emphasis is not on semantic dissection of communicative events (Potter \& Wetherell, 1987; Sacks et al., 1974). Neither does it rely on having a specific theoretical grounding in how it approaches making sense of meaning gleaned from data. It does however define a number of core practises to follow regardless of whether being driven from a theoretically grounded or exploratory basis (Braun \& Clarke, 2006).

\subsubsection{General Method of Thematic Analysis}

According to Braun and Clarke a good thematic analysis consists of 5 stages, some with reiterative processes within them making for a 15 point, idealised model of conducting thematic analysis, figure 2.1.1 shows an idealised process for a thematic analysis.

\begin{tabular}{|c|c|c|}
\hline Process & No. & Criteria \\
\hline Transcription & 1 & $\begin{array}{l}\text { The data have been transcribed to an appropriate level of detail, and the transcripts } \\
\text { have been checked against the tapes for 'accuracy'. }\end{array}$ \\
\hline \multirow[t]{5}{*}{ Coding } & 2 & Each data item has been given equal attention in the coding process. \\
\hline & 3 & $\begin{array}{l}\text { Themes have not been generated from a few vivid examples (an anecdotal approach), } \\
\text { but instead the coding process has been thorough, inclusive and comprehensive. }\end{array}$ \\
\hline & 4 & All relevant extracts for all each theme have been collated. \\
\hline & 5 & Themes have been checked against each other and back to the original data set. \\
\hline & 6 & Themes are internally coherent, consistent, and distinctive. \\
\hline \multirow[t]{4}{*}{ Analysis } & 7 & $\begin{array}{l}\text { Data have been analysed - interpreted, made sense of - rather than just paraphrased } \\
\text { or described. }\end{array}$ \\
\hline & 8 & Analysis and data match each other - the extracts illustrate the analytic claims. \\
\hline & 9 & Analysis tells a convincing and well-organized story about the data and topic. \\
\hline & 10 & A good balance between analytic narrative and illustrative extracts is provided. \\
\hline Overall & 11 & $\begin{array}{l}\text { Enough time has been allocated to complete all phases of the analysis adequately, } \\
\text { without rushing a phase or giving it a once-over-lightly. }\end{array}$ \\
\hline \multirow[t]{4}{*}{ Written report } & 12 & $\begin{array}{l}\text { The assumptions about, and specific approach to, thematic analysis are clearly } \\
\text { explicated. }\end{array}$ \\
\hline & 13 & $\begin{array}{l}\text { There is a good fit between what you claim you do, and what you show you have } \\
\text { done - ie, described method and reported analysis are consistent. }\end{array}$ \\
\hline & 14 & $\begin{array}{l}\text { The language and concepts used in the report are consistent with the epistemological } \\
\text { position of the analysis. }\end{array}$ \\
\hline & 15 & $\begin{array}{l}\text { The researcher is positioned as active in the research process; themes do not just } \\
\text { 'emerge'. }\end{array}$ \\
\hline
\end{tabular}

Figure 2.1: The 15 point process of a Good Thematic Analysis, adapted from Braun and Clarke (2006)

This is the generalised approach taken with both the two studies presented here; historical themes associated with Intellectual and Creative 
Work, and Public Submissions to the New Zealand Patent Act review. Both analysis incorporate a generalised thematic analysis approach as the guiding basis. A narrative approach was used for the historical and literature review study (J. H. Liu et al., 1999), which is even more exploratory in nature. This was used principally to gain an initial understanding of the contexts and to gain insight into how historical social representations and thematic fields might have been utilised by groups in the past. This allowed observation of how consequently these groups evolved to meet changes in technology, cultures and environmental constraints. This initial historical analysis also allows for a wider initial investigation of the more general nature of how knowledge and intellectual and creative work may exist as social representations in the everyday lives of groups, in many different contexts, and in a way the more focused second study would not allow.

The second study follows the methodologies used in the general theory of thematic analysis more closely, principally by relying on the use of themes that were extracted from the literature review of Internet and media discussions and historical accounts of Intellectual and Creative work in societies. To this end the primary technique employed is a tract labelling system, where multiple themes are attached to tracts from the submissions. In a similar fashion as is used in many massively collaborative Websites which follow design paradigms touted as Web $2.0^{3}$, one of these features allows users to tag content with arbitrary labels. This practise is fairly well established method in contemporary social science research under many guises, however the level at which feature of discourse is tagged/categorised varies from technique to technique. Other approaches which mark categories on smaller discrete units of content, begin from content analysis which uses a quantitative driven micro syntactical approach, tagging features at a word/grammatical level, then to conversation analysis which tags features of discourse (text/transcripts) at an event level, moving through to Discourse analysis which operates on a higher level shared meaning approach. My approach to tagging can

\footnotetext{
${ }^{3}$ See http://en.wikipedia.org/wiki/Web_2 for an overview of web 2.0 design elements and methodologies
} 
be seen as somewhere slightly above a discourse analysis approach, with my theoretical grounding being more varied than that of a strict DA reading which benefits from the sort of flowing multiparty discourses that a full DA treatment is best suited.

\section{Thematic Maps}

The use of thematic maps as a tool in Thematic Analysis is employed within both studies. The intial map of potentially occurring themes and groups is shown in figure 2.1.1 which was based on preliminary assumptions about what might appear in the corpus. This will be elaborated on and refined in a way that the themes are incorporated into the Social Representation concept of social objects. ${ }^{4}$ The initial themes as shown in the figure were refined during the historical literature review to contain the themes encountered there. This will be later used as the principle basis for thematic extraction tags for the Patent act review study. This refined table of themes is shown in table 2.2.1. Thus the mapping and relationship function that thematic maps have within thematic analysis methods have been replaced with a hybrid map incorporating social objects from social representations theory ( social object in themselves contain a number of themes ).

\section{Metaphor Analysis}

Metaphor analysis (Lakoff \& Johnson, 1980c) is another tool, which is not inherent to thematic analysis and is most often employed within conversation analysis techniques (Potter \& Edwards, 1999). Its usefulness in social representations occurs primarily within the initial anchoring and objectification phase of SR formation. By using metaphor analysis as a bridge between the two theories (CA and SR) it allows for a similar interpretation of how a particular metaphorical anchoring ( or mapping in metaphor analysis jargon ) can highlight certain features, thereby emphasising a particular theme or aspect of a target object that as the mapping applied to it, while at the same time hiding certain other features which the mapped

\footnotetext{
${ }^{4}$ The developed thematic maps can be found on page 83 and page 135
} 

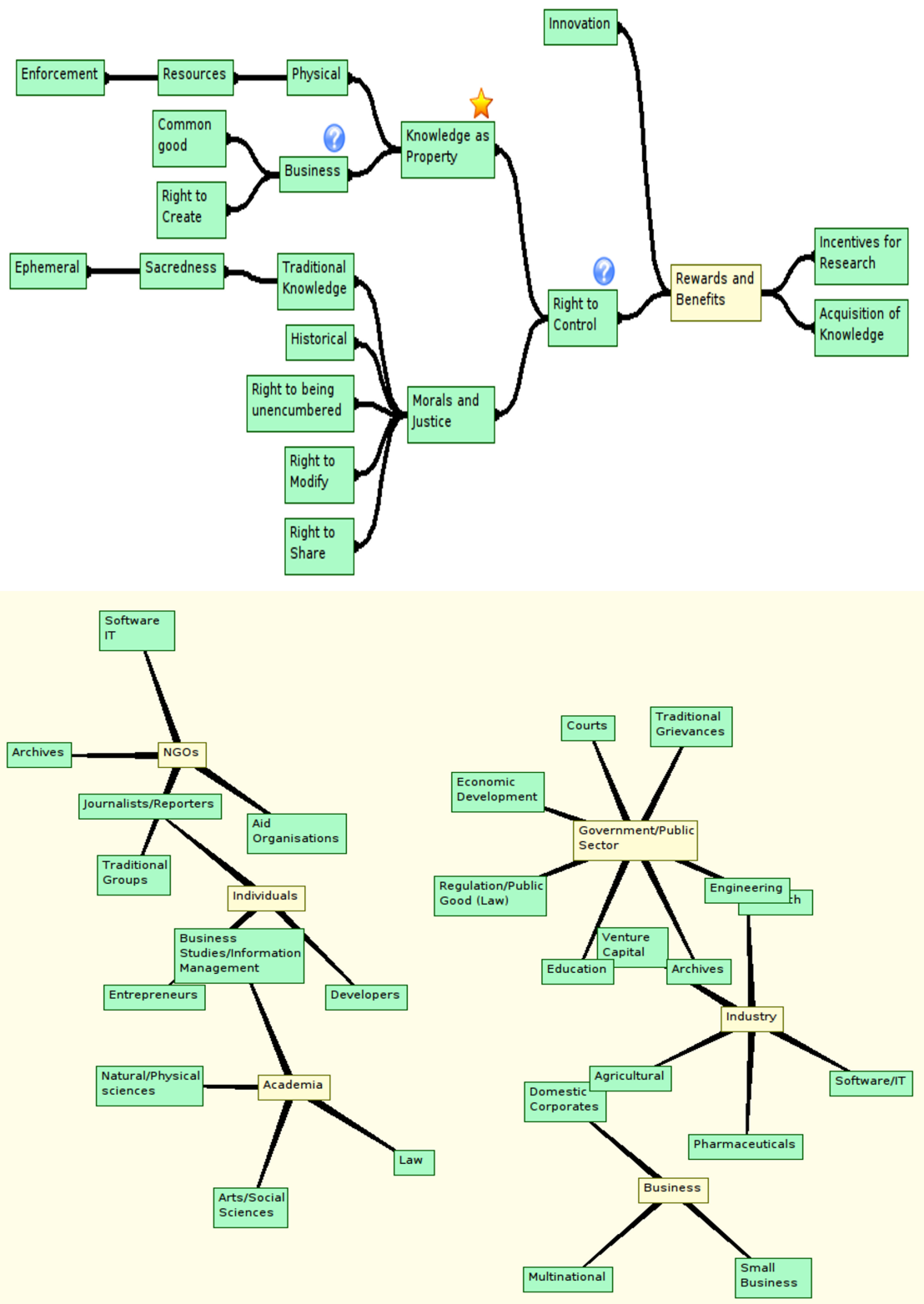

Figure 2.2: 2 Initial thematic maps of potentially occurring themes (top) and representing groups (bottom) based on reflexive understanding of the topic 
object may have because the anchored / reference domain does not contain any structure which resembles them. This mechanism is particularly useful when examining the social representation of intellectual and creative work, which in itself is a purely abstract object. As such it should be highly sensitive to metaphorical influence, especially if anchored to a physical domain.

\subsection{The Corpus}

\subsubsection{Historical Thema}

It would be impossible to start to interpret current representations without first looking to the past to see if there are patterns, cycles and themes which are analogous with current trends and discourses. To this end this narrative account provides an overview of global, societal and cultural examples of dialogues and historical events which revolve loosely around a theme of control of knowledge and creativity. This formed the basis for the thematic map which will be used in the primary data set of the patent act. Because it represents only a brief overview from secondary sources it cannot be viewed as a separate data set in of itself, more so a literature review of historical aspects which may help better understand current social objects in use around current social representations of intellectual and creative work. Broadly this data set can also be thought to contain a huge corpus of related material from Internet and Media Sources. This includes a number of popular technology and culture web blogs, legal blogs, main stream news sources, relevant documentaries and features, artists corporate and trade organisation discussions. Documenting all of these however is beyond the scope of thesis. Relevant material has been archived however and is available for inspection should any interested party wish to have a definitive list of these sources.

\subsubsection{The Patent Act 1953, 2004 review: Public Submissions}

The main data set is a corpus comprising of a mostly complete set of public submissions to the New Zealand Patent Act review. It includes public 
Table 2.1: Granular themes as encountered during the Literature review and Internet and Media corpus

\begin{tabular}{ccc}
\hline \hline & Themes & \\
\hline Freedom of knowledge & Human right to communicative freedom & Economic Inefficiency \\
Detrimental Effects & Incentivising Creativity/Innovation & Constraints on Creativity / Innovation \\
Coercion & National Mission & Global Mission \\
Natural Rights & Rewards & Public Good \\
Economic Rewards & Human Nature & Corporate Opportunism \\
Indigenous Rights & Trade Barriers & Harmonisation of Laws \\
International Obligations & Property & Business \\
Research & Developing Vs Developed Nations & Barriers to Entry \\
Education & Cumulative/Combinational Nature of Knowledge & Competitive Advantage \\
Individual Freedoms & Ease of Administration & Legal Precedent \\
Business Models & Measurement of Value & Historical Validity \\
Censorship & Control & Power of Knowledge \\
Authors/Creator Rights & Non-exclusionary nature of information & Property \\
Theft & Fear, Uncertainty and Doubt & Technological Challenges \\
Dominance & Exclusions & Specialist Markets / Industry \\
Traditional Knowledge & Prosperity & The Public \\
Censorship & & \\
\hline
\end{tabular}

submissions from each round ${ }^{5}$, and official summary documents as issued by the Ministry of Economic development (MED), the government ministry in charge of the review of the act. This act has still yet to be passed into law, and the final public submission round pertaining to the drafting of the act finished in 2005. There has been a further enquiry and review specifically on the issue of a research exemptions clause. The research exception round, which includes a public submission component, is not included in the corpus due to the enquiry document only being released by the Ministry for consultation after initial coding had begun. The last official document relating to the review process that is included in the data set was made available in 2006 and is a summary document from the ministry that purports to cover all discussion points from all rounds included for the final consideration process of the Draft Act. It provides the most authoritative official acknowledgement of the points which will be considered pending a final version of the proposed Patents Act 2004 legislation.

The data set comprises approximately 65 public submissions ( table 2.2.2 shows a full list of submissions ), and a number of related documents (such as summary and overview documents released from the ministry as well as the draft act itself). It is important to note that a number of the pub-

\footnotetext{
${ }^{5}$ The process of issuing a public review and enquiry beginning in earnest at the end of 2001
} 
lic submissions were not made available under the Official Information Act to the researcher as they were considered to contain compromising information pertaining to submitters or the companies they represented. The data set was coded for a number of criteria, table 2.2.2 on page 43 shows the criteria used to assess each document. Some documents were fairly substantial (the largest weighing in at nearly 200 pages) while others were short e-mails. A coding scheme that attempted to assess minute features obviously would heavily favour longer submissions in terms of reporting frequency. Because the goal was not to undertake an extensive discourse or content analysis only some language feature information was retained.

Table 2.2: Example of coding scheme utilised in assessing tracts of text, and examples of how this was used in analysis

\begin{tabular}{|c|c|c|}
\hline Example Tract & Coded for & Example of usage in analysis \\
\hline $\begin{array}{l}\text { For New Zealand, costs of patents are likely } \\
\text { to exceed benefits, as New Zealand is a } \\
\text { net technology importer. However, to not } \\
\text { award patents at all would involve risk and } \\
\text { cost: it would make New Zealand vulnera- } \\
\text { ble to diplomatic pressure and would result } \\
\text { in the loss of benefits of being a signatory to } \\
\text { TRIPs }\end{array}$ & $\begin{array}{l}\text { Stake, Obligations, Outcomes, Identity, } \\
\text { Economic/value imperatives, Compro- } \\
\text { mise, Negative opinion }\end{array}$ & $\begin{array}{l}\text { Support for construction of important } \\
\text { themes for the submitter (Economic). New } \\
\text { Zealand as society and being the primary } \\
\text { concern (Stake, Identity, Negative). Out- } \\
\text { comes of loss due to international obli- } \\
\text { gations i.e TRIPs agreement (Obligations, } \\
\text { Outcomes). Acknowledgement of harm } \\
\text { from patents system (Compromise, Nega- } \\
\text { tive) }\end{array}$ \\
\hline
\end{tabular}

\subsubsection{Internet articles and discussions}

There are many obstacles to using any corpus obtained from on-line sources, especially because of the diffuse multi-referential nature of Internet users. Determining any given site's referential and reader base is something that marketing agencies spend great expense trying to determine, in many cases without great success. Trying to collect an accurate profile of users of any given site, while quantitatively measurable through use of: geographic, user platform OS and browser choice, click statistics - the measure of how many links and what links on a given page any user in a single session uses; does not provide really provide any sort of accurate demographic or referential picture of any given site's users. This is further confounded by a large amount of non-human generated statistics, or incomplete/inaccurate data as a by-product of the Technologies employed, 
Table 2.3: List of submitters

\begin{tabular}{|c|c|c|c|c|c|}
\hline & $\begin{array}{l}\text { Round } 1 \text { - Submissions on } \\
\text { Discussion Paper }\end{array}$ & & & $\begin{array}{l}\text { Round } 2 \text { - Submissions } \\
\text { to Draft Exposure }\end{array}$ & \\
\hline Number & Submitter & Classification $^{a}$ & Number & Submitter & Classification \\
\hline 1 & Valerie E Looper & Patent owner & 1 & P J Burn & Orthopaedic Surgeon \\
\hline 2 & Ray Palmer & Unknown & 2 & Marisusz Nowostawski & Research Fellow \\
\hline 3 & Peter Andreae & Academic & 3 & Andy Elvey & Computer Programmer \\
\hline 4 & $\begin{array}{l}\text { New Zealand Association for } \\
\text { Animal Health and Crop } \\
\text { Protection (AGCARM) }\end{array}$ & Patent owner & 4 & Terry Cole & Individual \\
\hline 5 & John Parker & Academic & $4 a$ & Terry Cole & \\
\hline 6 & $\begin{array}{l}\text { Lewis' Barristers and Solici- } \\
\text { tors }\end{array}$ & Patent owner & 5 & Don McIntyre & CEO Lifevent Medical Ltd \\
\hline $6 a$ & BIO-GRO New Zealand & User & 6 & $\begin{array}{l}\text { New Zealand Open } \\
\text { Source Society }\end{array}$ & Industry Group \\
\hline 7 & $\begin{array}{l}\text { Women's International } \\
\text { League for Peace and Free- } \\
\text { dom }\end{array}$ & Humanitarian group & 7 & P L Berry \& Associates & Patent Attorney Firm \\
\hline 8 & $\begin{array}{l}\text { Crop \& Food Research Insti- } \\
\text { tute }\end{array}$ & Patent owner & 8 & David Shaw & Surgeon \\
\hline 9 & Tait Electronics Ltd & Patent owner & 9 & $\begin{array}{l}\text { Auckland UniServices } \\
\text { Ltd }\end{array}$ & Business \\
\hline 10 & Greenpeace & Environmental group & 10 & FICPI Australia & Professional Association \\
\hline 11 & $\begin{array}{l}\text { Pharmaceutical Manage- } \\
\text { ment Agency Ltd (PHAR- } \\
\text { MAC) }\end{array}$ & User & 11 & NZBio & Industry Association \\
\hline 12 & $\begin{array}{l}\text { Action, Research, and Edu- } \\
\text { cation Network of Aotearoa } \\
\text { (ARENA) }\end{array}$ & Humanitarian group & 12 & Fisher \& PaykelLtd & Manufacturer \\
\hline 13 & AJ Park & Legal practitioners & 13 & $\begin{array}{l}\text { Institute of Patent and } \\
\text { Trademark Attorneys of } \\
\text { Australia (IPTA) }\end{array}$ & Professional Association \\
\hline 14 & Telecom New Zealand Ltd & Patent owner & 14 & Adam Warner & Individual \\
\hline 15 & Adam Warner & Unknown & 15 & Baldwins & Patent Attorney Firm \\
\hline 16 & $\begin{array}{l}\text { Researched Medicines In- } \\
\text { dustry Association of New } \\
\text { Zealand (RMI) }\end{array}$ & Patent owner & 16 & Pipers & Patent Attorney Firm \\
\hline 17 & New Zealand Law Society & Legal practitioners & 17 & $\begin{array}{l}\text { Researched Medicines } \\
\text { Industry Association of } \\
\text { New Zealand }\end{array}$ & Industry Association \\
\hline 18 & ZESPRI Group Limited & Patent owner & 18 & $\begin{array}{l}\text { Management \& Technol- } \\
\text { ogy Systems Ltd }\end{array}$ & Business \\
\hline 19 & $\begin{array}{l}\text { FONTERRA Co-operative } \\
\text { Group }\end{array}$ & Patent owner & 19 & Morgan Read & Individual \\
\hline 20 & $\begin{array}{l}\text { Wellington Rainforest Action } \\
\text { Group }\end{array}$ & Environmental group & 20 & Robert McKinnon & Computer Programmer \\
\hline 21 & $\begin{array}{l}\text { Pacific Institute of Resource } \\
\text { Management }\end{array}$ & Humanitarian group & 21 & Watermark & $\begin{array}{l}\text { Patent Attorney Firm (Aus- } \\
\text { tralia) }\end{array}$ \\
\hline 22 & $\begin{array}{l}\text { Federated Farmers of New } \\
\text { Zealand }\end{array}$ & User & 22 & Ian Cockburn & Patent Attorney \\
\hline 23 & $\begin{array}{l}\text { New Zealand Institute of } \\
\text { Patent Attorneys }\end{array}$ & Legal Practitioners & 23 & $\begin{array}{l}\text { New Zealand Law Soci- } \\
\text { ety }\end{array}$ & Professional Association \\
\hline 24 & $\begin{array}{l}\text { Partner, Baldwin, Shelston } \\
\text { Waters }\end{array}$ & Legal Practitioners & 24 & PHARMAC & Crown Entity \\
\hline 25 & Glenn Fisher & Unknown & 25 & FICPI New Zealand & Professional Association \\
\hline 26 & $\begin{array}{l}\text { Nursery \& Garden Industry } \\
\text { Association of New Zealand }\end{array}$ & Patent owner & 26 & Ancare NZ Ltd & $\begin{array}{l}\text { Animal Health Products } \\
\text { Firm }\end{array}$ \\
\hline 27 & Auckland Uniservices Ltd & Patent owner & 27 & Alan Wilkinson & Computer Programmer \\
\hline 28 & Jay Ray & Individual & 28 & $\begin{array}{l}\text { New Zealand Institute } \\
\text { of Patent Attorneys } \\
\text { (NZIPA) }\end{array}$ & Professional Association \\
\hline 29 & Jane Penton & Individual & 29 & A J Park & Patent Attorney Firm \\
\hline 30 & Jon Carapiet & Individual & 30 & $\begin{array}{l}\text { Fonterra Co-Operative } \\
\text { Group Ltd }\end{array}$ & Dairy Co-Operative \\
\hline 31 & David MacClement & Individual & & & \\
\hline 32 & GE Free New Zealand & Environmental group & & & \\
\hline 33 & GE Free Northland & Environmental group & & & \\
\hline 34 & Ngatiwai Trust Board & Māori group & & & \\
\hline 35 & Chrystal Pitcher & Individual & & & \\
\hline
\end{tabular}

${ }^{a}$ Classifications are as they appear in official Ministry media releases and summary documents 
spiders - directory indexing agents, proxies and deliberate obfuscation by users. These all contribute to making quantitative analysis of any given web resource complicated. Further many sites do not publicly disclose site statistics, and many keep them closely guarded secrets either for privacy reasons, or for potential commercial exploitation. However like conducting readership surveys for newspapers, certain inferences can be observed from the articles, discussions and topics which appear on a regular basis. To supplement the wide literature review on the general topic of intellectual and creative work and societal control, limitations and freedoms,' a range of Internet websites were monitored regularly, and articles discussing topics such as freedom of speech, freedom of expression, copyright, trademarks, patents, court cases relating to these were reviewed. Any that included a comment and submission feedback section were snapshotted and stored for reference. The primary goal of this was to gain general insight into the various communities of practise which enter into discourse about the topic. This was not only useful for gathering themes for use in the Patent Act analysis but also in understanding which groups constructed. Both stake and the general themes of limitations of the topic. It also had the added advantage of keeping the researcher up to date with current legal, political and lobbying actions occurring around the world. While this data set has not been analysed in detail as a separate study it is implicitly included and used within chapter 3 .

\subsection{Summary recap of research goals}

While the methodology is loose by any account, the utility of this approach is that it allows for non-deterministic outcomes within the analysis that is required. This reflects the abstract nature of the topic of investigation, which concerns how ICW, ideas and knowledge, exist as social objects? Can SR theory foster a better understanding of how differing groups might create a common sense understanding for collaboration within a society? And have these social objects occurred through history, or are current representations as investigated through the New Zealand Patent Act Review submission fundamentally different? 


\section{Chapter 3}

\section{The Story of ICW and Society}

To understand the world around us people use histories and stories so that we are better able to frame our current environments and worlds. While this narrative process is not in itself a Social representation it does present a valuable opportunity to win arguments, highlight divergences and patterns around a topic. The problem with framing such an abstract concept as Intellectual and Creative work within a historical view is that it may be difficult to understand what it is that we are we examining. Do we look only at political and legislative controls in history, do we observe societal changes, do we draw on scholars and philosophy of knowledge and religious institutions, or do we emphasise technological change? The answer is a vague combination of all of the above, with a sceptical view that acknowledges those who write history seldom do so with an objective viewpoint. Those that try are only able to utilise the information available to them so in many ways must be viewed to have transferred a least a little of their subjective opinion into their records and narratives, knowingly or not. However, the traditions of institutional knowledge allow for a cumulative and incremental expansion of knowledge, but the knowledge must by definition take on a parochial taint (Bekar \& Lipsey, 2001). The concepts of collective symbolic history (J. H. Liu et al., 2005, 1999) incorporate this realisation somewhat, and try and draw on the narrative themes contained within the stories of history to point the way towards the importance of events as they are. Highlighted and drawn to attention are the stories as they exist within and between groups and societies today. 
As indicated within the opening chapter on social representations, for a social representation to exist it must draw on the social objects which are available to a given group first, and anchor to them. A social representation is by no means a stable construct. As in the Bauer and Gaskell (1999) model, a social representation can only be inferred in a single moment in time. The social objects that surround it and discourses which conceived it are all in flux as new information and discourse impact and become available to the individuals that comprise a group. That is not to say some are not more stable than others, but from a reductionist perspective like any quantitative variable if it is measured it is only valid for that moment in time, subject to the reiterative process of generation.

The topic which I define as Intellectual and Creative Work, encompasses what is referred to commonly in economic discourses as Intellectual Property, Knowledge Capital and intangibles, (Gowers, 2006) among other things. The importance of which is neatly summed up using only an economic perspective in the following extract from the Gowers' (2006) review on intellectual property in the United Kingdom.

" The increasing importance of knowledge capital is seen in its contribution to the value of firms. In 1984 the top ten firms listed on the London Stock Exchange had a combined market value of $£ 40$ billion and net assets of the same value. Advance twenty years and the asset stock of the largest firms has doubled while their market value has increased nearly ten times. The difference in value is accounted for by intangible assets: goodwill, reputation and, most importantly, knowledge capital" Gowers, 2006, p. 4

The questions this piece of information raises for me and for any partially interested observer of money and human endeavour, both personal and of the larger global kind, is this. If we are to for the moment assume that the United Kingdom is typical in regard to these trends as a developed nation, which for the most part it is, and extrapolate out to the world (Gowers, 2006; Yasuda \& Kato, 2007; Carlaw, Oxley, \& Nuth, 2006), when and how did people start to measure, what must have existed before (in this case knowledge )? And how have they come to place such great value as to justify a 9:1 ratio supplementary to measures of economic worth of what has been considered for the majority of recorded history the whole of 
capturable, quantifiable resources? Why have minerals, organic produce, land and all that is the physical realm of working these resources become such a fraction of capital in comparison to the new global market economy of intangible ideas?

In terms of social representations and related social psychological theory, which is what I am primarily interested in rather than the legal and economic mechanics involved, this abrupt change in the conception of Intellectual and Creative work even when just reflected within the subset considered by economic analysts is monumental. From a conception where many types of ICW are regarded as un-quantifiable to one where it clearly is, as demonstrated by the attachment of a market value, constitutes the single widest impacting event of recent history for its potential influence on what power, success and dominance are considered to be. This conception also implies that the price of a thought, the price of transmission of that thought and the price of reception of the same is all now potentially part of a market, somewhere. From humming a song you heard on the radio, to viewing an advertisement on the side of a bus, to playing a guitar - all fall under some sort of value paradigm. The potential when viewed from this economic perspective is that each of these has a market value, or multiple values attached to finer grained components of these behaviours. Where economic value is attached there must first exist a suite of control, rights and legal frameworks to necessitate stake, so what of these?

For the individual this is a vague amorphous and far away concept, the real question is, does what is reflected in the economic and legal reified world constitute a brute fact within a wider societal and cultural perspective? As I began, for any observer with a passing interest in money and the extent of human creative endeavour, which arguably is the majority of the world, it should if there is any validity to these claims of rights and value implied within the economic referent frame.

This is the economic narrative, the story of ICW as many states, corporates and non-government organisations tell. There are of course many alternative narratives many which try and work within the economic narrative which at it's core relies on free market efficiency (Benkler, 2002), 
and others either gloss, or reject the economic and thus property frame altogether. But all stories, no matter what embellishments the current discourses may have acquired in their current tellings, draw from the same bank of historical resources.

To continue the economic story to it's logical conclusion, does this brute fact of the commodification of intellectual and creative works serve to impact on the social representation of the creative and communicative world of the individuals that make up a society? At least from the referent domain of Law and Market Economics, Individual and groups must assume and somehow incorporate all the artifacts of intellectual and creative work that go into everyday experience within this market dialogue. Has this happened? Do people now evaluate their actions and exchanges within this market model ascribing the value, rights and legal duties that go along with it into how they see and interact with the world? What are the social objects that have come out of this? Where are the conflicts, and what is that elusive common sense understanding that underpins all of this? What were, or are, the existing social objects that are used to anchor this new paradigm? What are the rights, morals and duties that are found within the past, and incorporated into the now?

For each narrative story that exists around ICW these are the same questions that need to be asked. It would be foolish to assume any one is correct. To assume that the legal and economic referent sources are the sole proprietors of the reified way of how ICW should be measured would be to relegate whole cultures and epistemologies as worthless simply because they exist outside of the economic capital and the legal controls required to maintain the fence of rights that creates economic value.

At least for the last 3 questions in the preceding paragraph, looking at the evolution of the legal, socio-cultural and technological influences, and more so the epistemological traditions of the past may provide some insights into these questions, they also provide a starting narrative and story to begin to unravel the themes in current discourses, and whether a Social Representation of Intellectual and Creative work exists today. Or have world economies simply been gamed ${ }^{1}$ by stake holders to invent enclos-

\footnotetext{
${ }^{1}$ Gamed is a popular colloquialism, when something is gamed or by gaming a system,
} 
ing rights to create value for something that isn't there for the vast majority of people? If so then this should be reflected within the Social Representations surrounding intellectual and creative work as the social representations will reflect the attitudes and behaviours groups might hold in regard to ICW.

\subsection{Toward social representations of knowledge}

\subsubsection{Early Conceptions of Knowledge}

The endeavour of creating and communicating knowledge begins in prehistory, and may likely have begun in earnest with early tool building primates ( (Matsumura, 2006; Zimmer, 1995, 2007). The instruction in complex use of tools and other complex behaviours within groups is still observed within modern non-human primates today. Many of these complex behaviours have been demonstrated to occur only within certain social groups where these behaviours pre-exist in other members. It has also been demonstrated that the knowledge can become extinct when there are no remaining practising members to pass on the knowledge (Cox, 2000). In this context it is the rudimentary step from purely environmentally conditioned animal to one which may both receive and pass on complex forms of knowledge without direct experience. This forms the basis for ever increasing complexity and even more importantly, greater distance from personal experience to synthesised knowledge in its place. Here the 'Work' component occurs in two stages; the effort of communicating, and the effort of receiving, The work component is the process of investing labour into the communication of concepts. Importantly the process of storage of knowledge is abstracted, while there is probably work occurring to continue to keep knowledge accessible for the individual, at a group and social level a discrete unit of knowledge may become more amorphous especially when historical, social political and cultural contexts are incorporated. It is these complications that provide the institutional basis of control, appropriation and legitimacy. The old adage that knowledge

means to exploit weaknesses 
is power provides insight into how knowledge is valued for ability to influence. Various states, rulers and theocracies have imposed restrictions on forms and types of intellectual and creative work(s) which were permissible in a given society. And so the history of the institutionalisation of systems of control and rights surrounding ICW begins as censorship and as a way of controlling propagation of ideas. Until the advent of writing systems, however, most knowledge propagation would have likely been spread through direct individual interactions, reducing the need or effectiveness of any widespread system of enforcement and attribution. With the advent of writing systems decrees and policy became easily centrally governed and widely distributed leading to the advent of large pre modern era society.

In these pre modern era societies we see the first refined forms of specific philosophical traditions relating to knowledge (Hesse, 2002). Distinctly lacking is the concept of ownership from any of the pre modern societies. The Greeks, Chinese, Jewish and Christian worlds all disavowed any form of human ownership of knowledge. One anecdote of the extent to which knowledge was viewed as beyond the extent of human systems of valuation via money was in Greek society. Plato is documented as decrying of the Sophists who charged for instruction (Hesse, 2002). However manuscripts were still able to be traded and owned, but only in exchange for the physical labour involved not for the texts themselves. Within Chinese philosophy even stricter practises keeping knowledge and even the systems of communication (Alford, 1997) bared from commerce are observed. The concept that both the knowledge and the characters themselves originated from nature and from divine sources respectfully and thus could not be bought or sold. Confucian thought despised commerce and so with it writing for profit. The art was practised for reputation, self improvement and the esteem of future generations. Exceptions were made for incidental gifts of patronage however (Alford, 1997; Hesse, 2002). The system of bureaucratic power entwined within the Chinese socio political environment comprised mainly of educated middle class, the so called literati. This provided another means of institutional diffusion of knowledge, whereby scholars and political dignitaries routinely were ousted 
from power to subsequently again rise through various levels of society. This type of arrangement meant that a patronage system for scholars and teachers was well established in the form of restitution in China by the time printing was introduced (Alford, 1997).

Islamic cultures also did not practise ownership of ideas rather all knowledge was thought to come from God, with the Koran being the single most important work from which all other knowledge was derived. However because of the Islamic tradition of oral recitation being paramount in transmission of the word of Allah, the importance of the Koran (which literally means recitation) was as a tool for memorisation. This means it was checked against faithful oral accounts, rather than the reverse as in much western religion. This reliance on oration meant that the influence of printing technology was slow to influence Islamic regions (On Islamic Customs and "IP": Jakarta Post, n.d.; Kuran, 2001; Jamar, 1992). A notion of authorship did emerge eventually. However this was more a practise of ensuring reputation against fraud. An example to best illustrate the Islamic notion of authorship best describes the construction under Islamic law:

\footnotetext{
"A thief who stole a book, was therefore not subject to the punishment for theft - the amputation of his hand. Islamic law held that he had not intended to steal the book as paper and ink, but the ideas in the book - and unlike the paper and ink, these ideas were not tangible property." - Jamar, p. 2
}

A similar concept of knowledge existed in the Judeo-Christian world. Knowledge was a gift from God to be shared freely. Medieval theologians interpreted the biblical passages underpinning these ideologues and wrote them into cannon law doctrine: "Knowledge is a gift from God, consequently it cannot be sold" (Hesse, 2002). Selling something that belonged to God constituted the sin of simony. Doctors, Lawyers and Professors were all bound by this and prevented from charging for their services. However they might receive gifts for imparting the wisdom, in a similar manner to the Chinese construct. (Hesse, 2002). While the philosophical underpinning of intellectual and creative work being a taboo article (McGraw \& Tetlock, 2005; Golub, 2004) in commercial exchange and ownership was almost a fairly ubiquitous norm among cultures in the 
pre-modern age, this did not mean that knowledge flowed freely. Rather knowledge was proscribed, reproduced and censored by those agents whom were in some way 'ordained' with it's administration. This tightly controlled ideas and expressions and ensured they were intertwined with rulers and theocracies ideologies. In Europe the tight relationship between the roman-catholic and the various monarchies and emerging states, allowed the church the means to solidify an institutional agency. In the Islamic word the practise of religious law did the same and within China the bureaucratic agency.

\subsubsection{Comments on the treatment of knowledge in prehis- tory}

The various cultural accounts of knowledge presented above all echo one common discourse from which a social representation of knowledge can be inferred. This discourse is the one of "sacredness" assigned to knowledge. Essentially what seems to be the dominant discourse in early history is the conception of language and by inference all knowledge, as being divine in origin. This overarching "sacred" representation of knowledge seems to be non-culture specific. Within the context of this sacred representation, knowledge holders and repositories of knowledge become closely intertwined with whatever ruling doctrine was in power. Abstract notions of knowledge and creativity were essentially embedded within individual social roles and with those roles varying levels of affiliation with hegemonic powers. With the exception of the Greek academy, institutions devoted to institutional memory may not have been detached from in power ideologies (Bekar \& Lipsey, 2001). These institutional memories free from parochial traditions, Bekar and Lipsey argue, are central to $\mathrm{Cu}^{-}$ mulative and incremental expansion of knowledge. Diffusion was left to individuals who held knowledge and were valued not for the knowledge itself but for its practise. This formed the core valuation of an individuals intellectual and creative worth. This is best evidenced in the tradesman like quality of how knowledge is described, and how individuals were seen as intermediaries between the divine and people. Certainly innova- 
tors must have existed, but demonstrably that within the social representation that seems to be observed the concept of creator does not have invested in it any conception of ownership over the knowledge used in production, attributing it to divine origin. Based on the above observations any claim to proprietary over knowledge would need to based on some sort of claim to divine lineage, indeed this is well evidenced in both the Judeo-Christian tradition (i.e Ten Commandments) and the Greeks (with the practise of Oracles). Implied in the "sacred" representation is a commons conception of ICW, but lacking a method of effectively maintaining abstract knowledge at an institutional level apart from parochial tradition. ICW is represented as being diffused through practising individuals, mediated through the social structures obscuring what is otherwise a fairly strong discourse of "right to access".

\subsection{Early modern era and the advent of print}

\subsubsection{First attempts attempts at commodification of knowl- edge}

The advent of printing technologies first in China in 868 A.D ( the Diamond Sutra, a Buddhist scripture, is one of the earliest examples of dated wood block printed text) with block impression and then move-able type in $1041 \mathrm{AD}$ attributed to Bi Sheng (Alford, 1997) and the perfection of the technique later within the Korean kingdom of Goryeo would lead to profound social and cultural changes. China and surrounding areas were not anywhere near as influenced as Europe would be however. This has largely been credited to the ideographic script ( hànzì ) employed in the region which limited both the amount of written material, and the effort required to reproduce it. The ideographic script also meant that there were only an elite proportion of society able to read and write it, and learning it required much effort.

In Korea (which had been using the Chinese system of Hanzi up until this point) this recognition of the importance of developing a common, more widely accessible script, and the inherent problems with the ideo- 
graphic script, as well a political/cultural identity issues, led to the development of Hangul in the kingdom of Goreyo around the 1400s. Hangul from a written language perspective remains one of the most technically efficient, being able to represent the full range of human vocal sounds and most languages ( although in practice only a small subset is commonly used ). Because of its deliberate design goals of representing sounds rather than ideographic depictions ( phonemic) it was immediately popularised outside of its original intended purpose ( which was primarily as a simple writing system for women and commoners) (Diamond, 1999).

Around the same time as Hangul was being introduced in Korea the importance of accessible mode, as well as media, would be similarly echoed in Europe but in more subtle way as regional languages supplanted Latin as the lingua franca of written material, leading eventually to Latin's decline and an important factor in coalescing nations in the European block into more well defined countries by promoting and legitimising regional languages as being symbolic of national boundaries (Hesse, 2002).

The increase in literacy to come about by the codification of spoken languages into written formats would lead to previously inaccessible official and historical documentation, being thought to become more widely accessible, thus leading to the first attempts at censorship and control around information which had previously been tied to gatekeepers, scribes and bards. And with moveable type the technology of mass duplication was made available to societies.

In terms of control over the publication of literature China had already implemented legal codes banning transcription and publication of certain texts as early as the Tang dynasty (A.D. 618-907) (Alford, 1997; Hesse, 2002). With the advent of move-able type and printing an extensive regulatory framework was established around the print industry during the Sung dynasty (960 - 1179) with official governmental printing houses being established and granting state privileges over publication of certain ICW, such as almanacs; astrological charts and dynastic histories, to select imperial officials (Alford, 1997; Hesse, 2002; D. Rose et al., 1995). However these privileges were not property or ownership, but a grace extended from those in power, and as such were revocable at any time (Hesse, 2002). 


\subsubsection{Incorporating diffuseness into the SR of ICW?}

At this point the "sacredness" observed in early history was changing. With publication and literacy no longer as rare, the concept of controlled diffusion became very tightly wrapped into the social representation of ICW. Intellectual and creative works commanded a position in society where the benefits of distribution were more important than the concept of sacredness. Having viable mobilisers (namely publishers, translators and the social elite), allowed the separation of individual knowledge bearers from also having to occupy the role of knowledge maintainers and distributors.

This separation, amounts to a representation of a 'Proprietor', a vendor distributor and merchant of ICW as being key within the evolving representation. While the content and social representation surrounding it may have remained somewhat anchored to the 'sacred' discourse, the method of transmission became an integral aspect of the representation in itself. The state was comfortably able to assign a right that had never before existed to publishers, the right of reproduction. While this had existed previously within the various religious institutions, the rights to reproduce knowledge were carefully crafted rituals. The Chinese state's investment in publishers, both by helping to establish them and granting them privilege, was the first time secular society would be granted privilege in the same way religious institutions had. This would create precedent for state institutions to divest privilege over knowledge resources in such a way. It of course also created an implicit discourse that the state would police such matters.

The privileges seen here embody more a negative right than positive. Up until this time ICW appears to be constructed on the basis of an "allow all, deny some" rule chain of reproduction rights. In introducing a system of privilege to favoured publishers, and defining categories the rule set changed to a "deny all, allow some" rule chain. This created a market, not so much for the written material itself but for the privileges the state could grant over publication of materials.

While we can almost be certain that this representation of ICW existed only among those people who it applied to directly, (i.e publishers), it did 
create the precursor to a core concept later employed in Europe, which was the division of concept and reproduction, (or rather idea and expression) and that each could be treated as separate entities. This mediated the SR of ICW within a system of control rather than one of a marketplace.

\subsubsection{The division of knowledge in Europe and the influ- ence of Martin Luther}

The development of the European printing press by German Johann Gutenberg in 1453, some 400 years following the Sino-Chinese implementation, brought huge changes in the way in which intellectual and creative works would be valued. Traditional representations and conceptions about knowledge would be challenged. The printing press is arguably the second or first most disruptive technology yet invented. A disruptive technology is one that displaces or fulfils a need within a market where there is a existent system, in this case hand transcription. The term also describes the economic impact of the new technology (Christensen, 1997). The widespread adoption of the printing press in Europe firstly rendered many traditional forms of control ineffective, which relied on exerting control over the specialised labour force required to reproduce a text; notably the practise of monastic transcription, which a single monk could take as long as 20 years to finish a copy of a bible whereas a printing press could produce many thousands of copies in weeks. This changed both the status of printed texts from being luxury items held in high regard by only a wealthy and privileged few, to being items available to lower classes. Second it allowed individual authors to mass produce texts at little time and expense allowing for a wider range of topics to become available and for greater influence to be exerted by individuals and groups within society. Martin Luther's widespread dissemination of provocative ideas on the interpretation of biblical scriptures in the 16th century would be one of the first demonstrations of the power the printing press would have on the ability of individuals to influence society through mass media. Luther's excommunication by the catholic church and his subsequent exile for the rest of his life reduced none of his ability to publish and have his work dis- 
seminated widely throughout Europe. Arguably the Catechisms, Small "Guidelines" to various Christian doctrines, formed the basis of modern Christian faith, this in combination with his German translation of the Bible (the first translation of the bible into a local dialect) undermined the power of the priesthood to act as intermediates, putting scripture into the hands of the people (Haile, 1983). These pieces were written while in Luther was in exile but were widely distributed and reproduced via confidants.

These and other demonstrations of the power of printing presses to empower and alter control of social institutions and belief just after the advent of printing in Europe took advantage of the relatively unprepared authorities ability to deal with the new technology in an effective manner. It is unsurprising that following the introduction of the printing press, many European nations began to introduce more structured forms of legal and institutional methods of controlling who was entitled to publish works, and various rights of authorship and attribution that would accompany those privileges to publish. It is interesting to note that in the case of Luther that the terms of his excommunication also included a ban on his works. The so called "Edict of Worms" banned the reading or possession of his writings. However, sympathetic political connections spared Luther from a dire fate and hid him into exile. The edict was never enforced and the promulgation of the ban was most likely diffused by both the distributive framework made available via print and the pervasiveness and demand for his work, which meant profits for the printers and distributors at a time when concepts of ownership/authorship compensation did not exist. This further aided the growing popularity for Luther's ideas throughout Germany and Europe (Haile, 1983).

The demonstration of Germany's inability to adequately control and censor the advent of wide scale publishing reflected the complicated system of principalities and governance in the region. The republic of Venice perhaps was the first to introduce a legal system of controlling publication privileges in 1476. More a privileged monopoly, Venice granted exclusive printing privileges for the Venice region to individuals for a period of 5 years in 1469. This concept of granting exclusivity of printing privilege to 
individuals for a period time for particular cities, regions and or genre of texts spread rapidly first to other Italian states, and then to England and much later France (Hesse, 2002).

England's implementation of the concept differed slightly, and came in the form of royal grant in the form of the title of "Kings Printer" first granted in 1504 to a one William Facques (Hesse, 2002). This grant gave exclusive privilege over the publication of royal statues and proclamations and other official documents. By 1557 the crown had given exclusive privilege of printing and publication in general to the stationers' guild for all texts within the kingdom. In 1559, owing to the continued religious unrest within the English kingdom, Queen Elizabeth I ordered an injunction forbidding publication of any text unless it had been licensed by censors appointed by the crown. The stationers guild were required to keep 'copies' of the licences for each and every book it printed, these copies were in turn bought sold and traded within the guild members in exchange for exclusive 'right' of publication over those works. By the 1570's four prominent members held a majority of these licences of 'Copy-Right' over specifically the most lucrative books and genres within the guild, to which they claimed the 'letters patent' provided their own exclusive and perpetual property (M. Rose, 2003). In actuality these privileges were created for reasons of royal censorship and were a 'privilege' which could be revoked at any time by the crown. This system constituted of the first large publishing monopolies rooted in royal privileged monopoly grants. These privileges grants also were applied widely to other domains outside of publishing, the purposes of which were for control of the spread of religious ideology and other forms of ICW that may be against the state. This privileged system was to be adopted across Europe leading successfully to the inseparability of the commercialisation and diffusion of such ICW to the interests of the absolutist states attempts to control knowledge in their realms. 


\subsection{Interpreting privilege and Monopoly}

Discourses of control are pervasive during the initial period of European pre-modernity. So too do these discourses permeate the social representation of ICW. While not explicitly removing the divine discourse, by essentially expanding the diffuseness aspect states are neatly able to compartmentalise and legitimise expressions of knowledge in a way which would allow for it's control. This can be seen within the establishment of various systems to award monopolies over a huge range of ICW and related practises. The social representation at this point is maintaining the integrity of the divine aspect by not actually invoking a property discourse, but by giving power to state both to grant privileges and police those privileges. The treatment at this early stage is uniform, all forms of ICW are essentially incorporated into an exclusive monopoly grant like system (at least within large parts of Europe). Text and the written word at this point are handed over exclusively to publishers within this legal framework as a single expression of ICW (and therefore as a single grant of monopoly) as being just another form of trade monopoly, further implicating publisher's into an overseer and proprietor role over texts. This period is important, as the publishers' role as the maintainers, registrars and enforcers of censorship, becomes the most integral part of wider societal level representations of ICW. The second most important impact is on the legitimacy of trading in ICW, through the increased willingness to grant monopolies to secular society and the rise of economic discourse as an objectification for treatment of abstract notions as trade items. Publishers were able to successfully transform grants of privilege into commodities, within their own spheres.

\subsubsection{Of the Author}

But what of the author in this system? Authors still were unable to claim royalties/profit from their published work. Authors were free to sell manuscripts to publishers in exchange for a one time fee, but unless they were granted a royal privilege in their own name, any proceeds from the sales of their work went to the publishing guilds. For authors the value of publication 
was in the royal and aristocratic patronage that it might bring with its' publication. The concept of intellectual property non existent and inconceivable in the accepted theo-philosophical epistemology of the time.

The renaissance era beginning in the 1700's would bring into question many of the social structures prevailing throughout Europe and with it a new understanding and debate over the nature of knowledge would emerge from a varied range of parties.

The widespread availability and dramatic increase in literacy within the middle class during the 18th century saw a shift from intensive to extensive reading. This fuelled a burgeoning publishing industry with a growing demand for secular modern literature (Hesse, 2002). This would in turn lead to strains on a system of publication which was rooted in the idea of there existing only a fixed amount of divine knowledge to be known, transmitted and interpreted. This increased demand for secular materials of various descriptions; - theatrical plays, self help manuals, novels - would also increase the number of individuals aspiring to be writers. For the first time writers across Europe would begin to attempt to live by their pen rather than patronage, unsurprisingly demanding better remuneration (Hesse, 2002), these factors combined would bring into question the traditional concept of authorship.

Following on from these demands saw the rapid increase in 'Piracy' across Europe. If "no licence existed before publication" the rogue publishing houses argued, meant that the work was freely available to be copied. The most vocal and prolific of the rogue publishers first came from small disputed regions or across national borders, they also made available cheap reprints of popular works, widely disregarding the guilds privileges. They touted themselves as servants of the public interest by "freeing the public from the monopolies of the publishing guilds". Why? they argued, were the guilds privy to exclusive right over works of authors who were no longer living, nor for that matter works composed before the invention of printing? Would not the propagation of the enlightening works eclipse the selfish interests of individual publishers? (Vaidhyanathan, 2001). In revising many of the laws concerning censorship with the aim of increasing the educational levels within their realms, governments and states 
inadvertently would bring into question the entire system of privilege and grant of publication right. The disorganisation inherent in the old system within the burgeoning industry and a formulation of a replacement, would be primarily guided by a deep philosophical questioning on the nature of knowledge. With increasing frequency secular authors began to claim that they themselves were the originators of their work, rather than transmitter from a divine source. They were claiming their creations as their own property, and as such subject to the same legal rights as other forms of property. Demands that authors should not have to sell their works in order for them to be published became common, with many claiming that those were contractual rights up to the author to trade and use as they wished (Hesse, 2002). The publishing guilds hoped to secure perpetual rights in order to defend in court against publishing companies not complicit with the old system of grant of privilege. Publishers in outlying regions and cities railed against the monopolies of the guilds. These conflicting interests created a debate over the underlying epistemological nature and purpose of knowledge throughout Europe, over what on the surface appeared nothing more than a system of commercial policy (Nachbar, 2006). Indeed the first legislation to introduce some form of recognition to the rights of authorship, the oft referenced British Statute of Anne $^{2}$ (8 Anne c. 19, 1710; Vaidhyanathan) was in response to the monopolies which the publication guilds had enjoyed. The statute introduced in 1709 , would be the first legislation which conferred rights to authors, rather than publishers. The exclusive rights of authors were valid for a period of up to fourteen(14) years at which time it would pass into the public domain or if the author was still alive for another fourteen (14). For existing works not already in print it granted twenty-one (21) years. It also made explicit reference about the fact that authors and publishers could not restrict use after publication. Above all else the act was designed to give authors precedence over who they choose to publish their work.

Primarily championed by the publishers' guilds, after suffering from falling out of favour with the state and monarchy in the preceding decades,

\footnotetext{
${ }^{2}$ Full text of the original can be found on the wiki source project http://en .wikisource.org/wiki/Statute_of_Anne
} 
it was to be challenged almost immediately by the same factions in a collusive suit, designed to allow for matters to be taken through the common law courts, rather than legislative courts. The first attempt at this failed as the court discovered that the two publishers involved were working together to create a common law precedent for the treatment of authors conveyed rights as a form of property, and as such that there were perpetual rights of ownership.

A real case, challenging the statutes twenty-eight (28) year term occurred later in 1769. A publisher had bought the rights to an poem called "The seasons" and had enjoyed the exclusive right to publish it for the full fourteen (14) years plus fourteen (14) year period under the statute. It lapsed in 1769 and another publisher printed copies, and subsequently was taken to court by the original publisher (Vaidhyanathan, 2001; M. Rose, 2003) This case was won by the original publisher, setting a precedent in common law. By using property theory and law to apply to ICW, in particular the concept of perpetuity of ownership was established. The publishers were understandably happy, however in 1774 after the rights for "The seasons" had been on sold to another publisher Beckett, and one Scottish publisher Donaldson would challenge this ruling, by publishing an unauthorised edition of the seasons. Donaldson would be subsequently taken to court by Beckett, and would successfully appeal taking the case to House of Lords, who ruled that their was no such thing as copyright in the common-law, effectively removing and securing the role of what would come to be called the Public Domain.

It is interesting to note, that this case famously is the first to appeal to some conception of the public domain. While the discourse of the 'public domain' does not enter into use until some time later, there is a strong negative right of anti-monopoly which overshadows most of the legal 'talk' at the time. Indeed the statute of Anne and the interpretation and confirmation in the Donaldson vs Beckett case demonstrated that anti-monopoly discourse was a strong argument against what is essentially a economic positive right argument on the side of the authors (and to which was championed by the publishers after loosing their monopolies over publishing). However even at the time of the Donaldson vs Beckett decision 
there was disagreement within a panel of judges asked to advise on the case (M. Rose, 2003).

The continued argument over the nature of copyright and authorship would continue well into the 1800 's, with many notable scholars championing aspects of the philosophical underpinnings surrounding ICW. Arguably this progression would culminate in the Berne convention in the late 1800s at the start of the modern area.

\subsection{The trinity of Representation: User, Creator and Proprietor}

The period covered above is large, but it represents perhaps the most important transition towards modern social representations of ICW. There becomes a clear separation in the treatment (at least in England) between textual ICW such as; - Literature, Encyclopaedias, Dictionaries and Journals. The main driver for this was historical, with the separation existing as a result of a censorship system of registration, which was adapted into a trading system by the publishing houses. This separation became a convenient for detailing the rights of reproductions of individual texts, and was legitimised by the state when it allowing the publishing guilds to keep current monopolies of privilege over publications when it abolished all others. The convenience is eventually transformed into it's own legal discourse of 'Copyrights' ${ }^{3}$, which until this point had not been legally distinguished from the grants of monopolies. This is the first real introduction of the "rights" repertoire into the ICW representation, and produces a discourse anchored in an individual notion of rights. This introduction and adoption into wider social representations, perhaps is the most significant event. The introduction of an objectified notion of rights rooted in an economic discourse was initially championed by the publishing guild to allow trading of grants of privileged amongst members. However the individual notion of 'rights' implied in this representation is picked up dramatically by authors.

\footnotetext{
${ }^{3}$ It is interesting to note that the term Copyright does not actually appear in the statute of Anne itself
} 
The entry of authors to the debate for the first time seems to be product of the artificial separation of ICW into Copyrights and "other". This gave authors the ability to be able to target a single legal construct to introduce new discourses of "natural rights" into the ICW representation. These "natural rights" discourses reflected a dramatic shift away from the "divine" SR. Essentially authors were arguing that they were creators of new ICW and as such "owned them" as a natural right. This discourse marks the entry of the property discourse from a completely different referential base. While property had entered the ICW representation some time before it had been in the form of ownership over grants of privilege. The authors were introducing the notion of property from a common law perspective and as such inject many of the discourses surrounding physical property into the social representation. This anchoring to property discourses provides the single most accessible reference frame for the "public" in the continued evolution of ICW representation.

A by product of this property discourse, was in the creation of a market based repertoire detached from publishers interests. This "readers" discourse (Chartrand, 2000) is echoed in the various state proclamations which included statements which referred to the good of society, and in particular how monopoly discourses were introduced for legitimacy of policy. The construction of "readers/public" was also invoked by authors themselves within counterarguments. The role of dissenters i.e. authors opposed to the "natural right" discourse and to a lesser extent publishers whom did not follow legal doctrine (constructed for the first time as "pirates" during this period) were instrumental in the production of this representation. Both appealed to representation of the "right to access" along with creating a new complementary discourse of "social nature" of knowledge. Both of these complemented the "divine" nature of knowledge representation. Importantly these contra arguments more explicitly created the "public" in a way as benefiting from relaxed ICW controls. The notion of the "Public domain" is for the first time championed detached from the divine implication of right to access, but rather invested within a "public good" discourse. The "social nature" discourse strongly reflects this, and creates the "public/reader" in a way which is not just one of a 
passive receiver but also as a transmitter as well. This varied significantly to the mainstream publishers' conception of the "public" as being passive receivers.

While the expansion of the various roles afforded to actions within the social representation of ICW led to a representation which invoked powerful property based discourses, this was never legitimised by institutions. In the British case it was strongly negated by ruling bodies, which would slow the transformation of ICW as being sacred or social in nature to being vested within a property discourse. However the expansion of the property and market discourses would, by the start of the modern era, be pervasive and while the social nature of knowledge debate would also prove to remain popular, it would become less and less represented within institutional forms of legitimacy.

\subsection{Patents}

The system of patents essentially has remained unchanged, from its' initial conceptions first encountered in late 15th century Italian origins (Drahos \& Smith, 1999). In England the 1623 Statue of Monopolies ${ }^{4}$ would be the first system of patents to be widely used, replacing the "Letter Patent" grant of privilege that required inventions to be registered with the crown before receiving legal protection (Nachbar, 2006). However this system, implemented during the rule of James the I, was widely criticised and abused as simply a way of extracting tax. It and was open to abuse from the crown granting patents over all manner of goods, and it suffered from this abuse during James I's reign. Following these criticisms the crown was forced to declare all current monopolies void (with the exception of the publishing monopoly afforded to the stationers guild), and to put limits on what a patent could be granted for. This led to the clause of patents only being granted for "projects of new invention". It would be revised under the rule of Queen Anne that any patent application must be accompanied by a description of the invention. The actual wording of the statute of monopolies from the rule of Queen Anne (reigned 1702 - 1714) and remains

\footnotetext{
${ }^{4}$ Full text available from http: / wikisource.org
} 
for a large part the basis for current patent law in both Australia and New Zealand. In particular section 6, which includes the phrase " manner[s] of new manufacture... [by] inventors".

The system for patent applications involved submission of ideas and inventions to some form of state institution, lodging a mock up or description of the device with an institutional power and in exchange for this disclosure be given a monopoly over the production and use of that device or method. The wording in the English statute of monopolies belies the general atmosphere of mistrust in regard towards granting monopolies and also in the scope to the type of application which a patent may be granted. In reference to scope, the "method of new manufacture" represents the technological advances starting to take shape before the beginning of the 1st industrial revolution (Bekar \& Lipsey, 2001). The majority of the act makes reference to the illegitimacy of existing monopolies and to the exclusivity granted. Implicit within the act is the understanding of the harmful effects of monopolies. The original length of grant under the Statue of Monopolies was limited to fourteen(14) years, although under the earliest Venetian system this length was 20 years, matching many countries' current length of patent. The importance of the statue is echoed throughout the majority of the Anglo-western world, with most colonies using the statue as the basis for their own versions (Vaidhyanathan, 2001; M. Rose, 2003). It is used reflexively throughout most modern debate as the basis for state based regulatory practise. Nachbar (2006), goes to lengths to explain the influence the statue has had among discourses of economic control against a free trade and market perspective and surmises that while it is used and referred to commonly as "the" economic guideline in discourse regarding monopolies, its' importance is more in terms of political accountability. The institutional aspects of control and accountability within the framework of legislation involving ICW was somewhat diluted by the economic undercurrent that the institutional controls created.

With the guidelines for the granting of monopolies established through the referent authority of the crown, the interpretation has varied little in the various localised versions. The history of patent law in America well reflects the nature of the English statute. Even more so, the original Amer- 
ican patent bill as implemented by Thomas Jefferson goes to great length to distinguish the limits of patentable material. Jefferson is well known to have been within the Condorcet tradition of assuming knowledge as being social in nature and that it's diffusion was not to the decrement of the originator. Jefferson's perspective was recorded in various places as such :

"ideas cannot be property" ... "there can be no natural right" ...

Society may give exclusive right for "convenience of the society", in fact, "ingenuity should receive a liberal encouragement" - Thomas Jefferson as cited in Vaidhyanathan (2001, pp 23)

Thus America's patent policy from the first patent law put into effect in 1790, (although Patents and Copyrights are explicitly included in the American Constitution itself) included many provisos to ensure that when patents were granted they met a number of criteria. These criteria included a test for novelty, and for submission of a model of the invention.

Unlike the British patent law, the context in which it was introduced was quite different. The original Statute of Monopolies was introduced to explicitly remove individuals and organisations from the seats of power they had previously occupied and so was introduced in a context of economic monopolies being rampant over various forms of manufacture and to ensure that any monopolies granted would lapse after a period of time. American patent policy reflected a greater emphasis on encouraging innovation. While as previously mentioned the economic nature of the Statue of Monopolies tended to be more pronounced, any reading of the American patent law as being an economic control however would be far less effective. The nature of granting patents under the original American policy was rooted in benefiting society as a whole, and providing only encouragement for innovation. The Statute of monopolies corollary to this was implicit in the limited duration granted over the method of new manufacture.

\subsection{Setting a standard for monopolies}

The social representation of ICW before copyright had existed was within a system which largely also regulated all other forms of trade. When copy- 
rights became part of the discourse, everything outside it essentially fell into an all inclusive category of 'other' ICW. Patents, while pre dating any specific copyright legislation, existed as simply a way of granting a monopoly. When the practise of granting monopolies in a way akin to taxation and cronyism was abolished in England, a standard was set which would apply to new monopolies which could be granted. This standard was 'method of new manufacture'. This relegated monopolies to only exist within the realm of ICW. This in combination with the distinction afforded through copyright discourses reflects a social representation which pre-supposes that knowledge and innovation is scarce. This is reflected in the protectionist stance under which patents are implemented. Innovation is anchored to economic incentive discourses within the patent system. While the ideal of increasing public benefit is well represented, it is done so within this core discourse of "innovation is scarce" and therefore requires incentive and encouragement. This 'innovation is scarce' discourse at this point is nascent, being heavily counter-weighted by public good and social nature of knowledge discourses.

\subsection{The Modern Era}

Industrialisation of many sectors of the manufacturing industries across Europe in the late 1700 and 1800's would create new challenges for creators of ICW. These challenges would be emphasised in the shift from ICW law debate being almost exclusively the domain of publishers and authors, into something more accessible and relevant to wider industry. The push came from sectors such as textiles and engineering, which were feeding the rapid growth of many other industries. No longer were copyrights as important as the right to monopolies over ideas and methods. For engineering and textiles this was more relevant than most. Competitors could easily dissect the workings of a machine and replicate its function. Patent law burgeoned with the "letter Patent" or simply "Patent", giving monopolies not just over the expression, but also over the idea itself. For engineering applications this was precisely the protection required to prevent competitors producing equivalent machines and under- 
selling the original. Thus the industrial age would be marked by flourishing patent environment spurred by technological and manufacturing innovation. The systems of Copyrights and dialogue of authors rights championed by philosophers such as Locke, and Hegel (Locke, 1838; Hesse, 2002; M. Rose, 1995, 1996, 2002b, 2002a), and the counter discourse of collective and social knowledge led by Condorcet, would influence the various cultural and social changes occurring. The French revolution in 1789 (Hesse, 2002; Khan, 2002) as a particular example, after which time the French would legislate similar protections to that in England. After operating for a long time under a titles and patronage system, to reward authors and intellectuals.

The establishment of overarching institutional controls would already be in place in the form of patents would seem to be supplemental to these academic discourses, with the main focus of debate firmly centred around authors rights, and probably not making such as distinction between copyright and patent as is made today in terms of the philosophical debate over the nature of knowledge. These authors may or may not have appreciated the already established system of institutional control regarding short term monopolies as established in patents. This omission is quite telling of the types of application to which patent policy was invoked. ICW related resources during the pre-modern era implicitly and clearly being defined within the emerging discourse of Copy-rights, while patentable material ( after the statute of monopolies) relating primarily to methods of "new" manufacture. When viewed within the technological environment of the 17th and 18th century's these methods of manufacture clearly were meant to be related to devices mostly involving primary production and physical methods. This was true both for American and British derived systems, it is not until relatively recently that the distinction between the two domains of coverage that copyrights and patents had become blurred.

The boundaries and limitations to which individuals could claim some form of institutional protection and rights to ICW, be it patent or copyright, and to a lesser extent other "soft" rights such as trademark and trade secrets starts to become contested during the expansion of trade boundaries. Copyrights and more so patents up to that point had existed within 
the vacuum of various territorial states. Within Europe, as cross border trade in goods and publications become more widespread, the need to establish legal frameworks for mutual trade success became paramount. Ensuring a stable economic environment also meant requiring a stable political and legal system to remove the free rider $^{5}$ problem. Drahos and Smith (1999) suggest that viewing systems of trade from this proximal basis produces a three stage process regarding the diffusion of the concept of Intellectual Property. It is important to note that Drahos and Smith's entire treatment emanates from a supposition of ICW as "Property". As demonstrated at least when viewing the pre-modern era, it is most likely erroneous from the perspective of social representations, lacking either a strong anchoring body of knowledge, or an objectified discourse within society. Indeed, institutional knowledge (in the form of legislation) prevented it from being viewed as such.

The following is an adaptation of Drahos and Smith (1999) three stages

Territorial: Dominated by boundaries of state and sovereignty with institutional frameworks being distinct from country to country. Holders of rights in one state did not have the same set of rights in another. Diffusion of ICW and 'free riding' across boundaries.

International: Increased interest in trade amongst nations. States entering into Bilateral agreements with other states. However states which perceived themselves as recipients of positive externalities remained isolationist. Increasingly complex agreements, leading to the establishment of institutions to negotiate multilateral agreements i.e. International Literary Association, BIRPI, WIPO

Global : More members nations joining institutions set up to facilitate multilateral agreements, leading to complex needs from a wider range of countries in varying stages of development. This initially created

\footnotetext{
${ }^{5}$ In economics, collective bargaining, psychology and political science, "free riders" are actors that consume more than their fair share of a resource, or shoulder less than a fair share of the costs of its production. The free rider problem is the question of how to prevent free riding from taking place, or at least limit its negative effects.
} 
an environment where many developing nations could gain access to copyrighted / patented material at reduced expense. Eventually this created dissatisfaction and bilateral embargoes from leading nations on member states which were believed to have too relaxed Intellectual Property laws. Eventually this would lead to the TRIPS agreement, and establishment of WTO.

Drahos and Smith's stages closely resemble the distinction made here between pre-modern and modern era. It is interesting to note that the transformation from political and social discourse to an almost purely economic discourse is legitimised by the establishment of institutions which exist outside of state spheres and polemics thereof.

Given the time line implied, the beginning of the International age begins after the French revolution. One of the first decrees they highlight recognising another state's system of ICW appropriation of control, would be a French decree of 1852 granting copyright protection to foreign works and foreign authors without the requirement for reciprocity (Drahos \& Smith, 1999; Khan, 2002). This was unprecedented and while England would have acts passed before this time $(1834,1844)$ these acts only granted reciprocal protection to acquiescent states. The impetus for England's early adoption was in response to the publishing environment in the newly formed Untied States of America.

America as already noted had adopted in it's constitution the ability to grant patents and copyrights "but only for a limited time", so that "the public may benefit from the fruits of Science and the useful Arts" (American Constitution). This incentive discourse pervaded the evolution of copyright in America. The history of American treatment of copyright provides an excellent example of the type of environment described in Drahos and Smith's international stage.

By the time the first lobby to extend the original 1790 term of copyright from fourteen (14) years (with fourteen(14) years renewable if the author was alive) appeared in 1831 . There had already been some extension of material that was valid for copyright. The successful lobbying led by Noah Webster (a prolific writer whose dictionary formed the basis of American English) who had campaigned for the first state based protections even 
before the constitution in 1790, would increase the period of copyright in the US to twenty(20) years renewable for an additional twenty (20) years. This duration would remain unchanged until 1909.

This process of increasing the scope, and extending the period of protection would repeat itself in almost all parts of the world. So would the recurring theme on which it is argued from, the individual/author/creators rights discourse which almost every case for extension up until late in the 20th century was based (Vaidhyanathan, 2001). Thus if considering the dominant social representation it is quite clear that by the mid 1800 's, at least within the realm of literary copyright, the process of anchoring and objectification is well established of the "authors" bundle of rights. However the argument that the anchoring was tightly mapped to property discourses is perhaps more contentious than inferring that it was more understood in terms of "creating" or "work" of an individual. Chartrand (2000) makes reference to this myth of an authorial "creator". While the act of creation implies ownership, if the property discourse were as implicit within the objectified social representation of ICW then the perpetual term and "natural right" nature of copyrights, first encountered in Beckett vs Donaldson (and in a similar case in America in 1934) would have been successful in influencing policy. The outcomes of these cases however has not been consistent with current legal directions however, as almost every lobby group contesting term length of copyright has argued for perpetuity. While this approach has been successful for increasing the length to, in the US's case, a period of 95 years. As for current policy, it has failed to ever confer the perpetual copyright sought by authors, in the US or elsewhere (with a scattering of exceptional cases such as the copyright on Peter Pan). Returning to the international situation of institutional forms of ICW protection, America found itself on the receiving end of a very positive number of externalities, namely the huge amount of British and European literature, all of which in American early copyright policy was afforded no protection. Thus publishers trading in cheap copies of popular European works flourished, making available classics and current works alike at prices affordable to a much wider proportion of the population. British and European authors were of course upset at this practise and 
England called for a Trans Atlantic copyright treaty to be put in place. (Vaidhyanathan, 2001).

America refused. The justification was threefold; that the value of the knowledge made available was of greater good, - the economic worth was better vested in the burgeoning publishing industry as afforded by defacto protection piracy granted, and there was no "natural" right of intellectual property in ideas. Of course publishers during this period had the best seat in the gallery. Not only could they publish highly sought after works, without any royalties exchanging hands, they could also publish American authors, many of whom at the time were the publishers themselves, under copyright protection.

These attitudes would change quickly once American authors started to gain popularity and number. As writing became a more popular occupation the authors began to question the effects of the proliferation of cheap European books. Not only were these works competing directly with there own, they would almost definitely be selling cheaper. This added to fears of losing the fledgling American cultural identity in the flood of European influence brought in through literature. Authors began the first wave of backlash against the copyright environment and joined with European authors to lobby for change. The publishers were not happy about the renewed interest in creating a trans-Atlantic copyright treaty. They had a lot to lose, especially the numerous publishing houses which specialised exclusively in cheap European reprints. The publishing houses however had a much stronger set of arguments in their favour against the authors decry of unfairness to foreign authors and establishment of a US body of literature. Despite numerous attempts the authors would be unsuccessful in lobbying for a trans-Atlantic treaty for nearly an entire century. It would take a collapse of the publishing houses collusive system of price fixing, ironically a result of "upstart" cheap publishing houses not adhering to the "courtesy system" of established publishers' practise of price fixing, before the bill would see fruition. In 1891 the British-American trans-Atlantic treaty was passed affording the same protections to British authors' works. This signalled the start of a new movement in the expansion of copyright term championed almost exclusively 
by the same author who had in his early career benefited from the lax protections afforded to foreign authors, Mark Twain AKA Samuel Clemens. Clemens once again invoked the natural property discourse and asked for perpetuity or at least a term of author's life plus fifty (50) years (ironically almost identical to the current American law). Clemens did not get what he wanted before dying but the term of copyright was extended once again in 1909 due in large part to his efforts. It was not to the extent he had argued, due in large part to publishers political clout (Vaidhyanathan, 2001).

Europe during the same period of time had been establishing multilateral agreements between nations. The realisation that continued bilateral agreements were both exhaustive and complicated to maintain and enforce lead to the culmination of authors and publishers groups to form institutions devoted to the proliferation of harmonised protections between states. The end result was the Paris and Berne conventions in 1893 and 1896 respectively. These conventions covered the two main bodies of ICW controls with the Paris convention" for the Protection of Industrial Property" covering broadly patents and trademarks and the Berne convention "for Protection of Literary and Artistic Works", broadly covering copyrights. Both of these documents would become legitimising historical institutions in themselves. Each document provided definitions of both the scope of ICW included, and baseline controls that signatory nations agreed to uphold. For both the most important is recognition of foreign works being subject to the same laws as the interpreting nation.

\subsection{Solidifying Institutional Legitimacy of Prop- erty Discourses}

The Paris convention revealingly makes exclusive use of the term property in reference to ICW. This treatment of patents as property and specifically when combined with the industrial prefix implies that the social representation involved in the patent system is anchored within industrialisation concepts and processes. This supports the previous notion that the evolution of the patents system was primarily driven by the industrial revolutions (Bekar \& Lipsey, 2001), and that by the time of the second industrial 
revolution in the late 1800's the social representation of patents seems to be well fixed within the industrial process representation. The centrality of big industry to the concept of the patent at the point the Paris convention was created enables speculation over public perceptions. This "industrial" concept of the patent system to this day is somewhat evident in popular culture portrayals of innovators getting rich by selling their patents to big industry or indeed the perception that anything patented is better than the competition. While these are not immediately provable as being the end objectified result of the anchoring of the social representation of patents to industrial processes, it seems that there is at least a degree of anchoring, if not some objectification involving industrial processes in the social representation of the patent. The fact that patents are so intertwined in this process is a clear indication that transformation of ICW into property discourses has well and truly become a baseline argument (Potter \& Wetherell, 1987). Any 'social nature' and 'public good' discourses are implicitly represented in the form of term and duration of patents. The social representation of 'innovation is scarce' is far more progressed within modern SR's of the patent system, and is the largest single source supporting continued existence of forms of institutional protections. Also complicating the patents social representation is the cross pollination of the creators/authors discourse from the authors and copyright debate. This is expressed throughout via the inclusion of the property discourse and is incorporated within the 'innovation is scarce' discourse by proxy of its pervasiveness. It is, however, most strikingly encountered within the economic discourse pervading the property discourse. This effectively is legitimised in the SR of patents and excludes any supposition of multiple independent or social producers of innovation or indeed ICW.

Returning to copyright discourses, the Berne convention (covering copyright) makes no reference to ICW as property. In fact the Berne convention goes on to make explicit the idea/expression dichotomy within copyright discourse. While it prescribes a minimum period of duration for copyright, it does not imply any natural rights nor property discourses. Thus the social representation of copyright in the modern era provides a clear distinction that an idea is social and can be independently created 
by multiple independent sources and is inherently 'social in nature', and that only individual expressions can be protected or have rights associated with them.

This produces a paradoxical social representation of ICW within the dyad of copyright and patent in the modern era. On one hand patents represent exclusive property discourses, objectifying ICW in a way which creates an imaginary scarcity and exclusivity so that it may be protected and valued in way which a physical resource may be, all so that it can be viewed as a viable commodity. While on the other hand, copyright acknowledges the non-physicality of ICW through the separation of idea and expression.

\subsection{Conclusion of the modern era}

With the Berne and Paris conventions homogenising most of the world's practises in regard to treatment of overseas produced ICW and with more and more countries joining the conventions through the 20th century, the emphasis on incentive and creators rights became subtly shifted. No longer were these rights in dispute at an institutional level, and the incentive discourse was now at this point a baseline argument for justification of the entire system of ICW control. While the period of protection would continue to be debated as before, it no longer held the impetus it once did during the initial debates. For a large part the 20th century would all be about scope, such as debates of pharmaceutical and chemical patent eligibility. Where would software fit into the system? Electronic devices? All of these are interesting and valid discussions in their own right, but do not add much to the debate save for the evolution of legal case history, and a rehashing of now familiar cycles and discourses.

Two events however do add value to the construction of ICW control and ownership, mainly because they demonstrate that problems with the system which have only recently become popularised notions, but occur during the first half of last century making them somewhat exceptional. The first is the aviation industry. Just prior to the first world war, the avionics industry was at a standstill, mainly because patents were be- 
ing used to stop competitors from producing important aspects of planes (namely a patent held over the wing design held by the Wright brothers). Following the commencement of WWI the government intervened and forced automatically cross-licensing to allow competitors to produce planes. A similar theme is echoed in the Radio industry but rather than a single patent being held by a single entity, it was multiple patent held by multiple entities. Patents covering important parts aspects of the radio were owned by disparate companies, producing a situation where none of them could produce viable products, eventually government intervened as it had done with the avionics industry. This time because of the commencement of World War II (Perelman, 2003[a]). While these are unremarkable to the observer they illustrate clearly that the current systems promise of incentive for innovation does not operate as clearly as it is represented.

Perhaps responding to these and other concerns, four independent and prominent scholars during 1930 - 1950 published articles regaling against the current system of patent and copyright. These scholars argued from varying points of view and speciality for what at the time was perceived to be to varying degrees a socialist system of administration of the ICW systems of control. Driven by concerns of the economic viability of the current system along with suspicion over the treatment and continued growth in scope of coverage of the institutional controls as applied to what one of them termed as "cultural knowledge". These four Arnold Plant, Robert Coase, Michael Polanyi, and Norbert Wiener essentially were at the heart of a debate over cultural property that existed during the first half of this century. A debate in which has seen their work reinvigorated in the past decade (Benkler, 2000, 2002; Hart \& Moore, 1990; Boldrin \& Levine, 2008).

The reason for including these anecdotes about the conclusion of the modern era, is that the concerns being expressed now were at least in part represented by a similar debate spurned by the privatisation of society, and the information monopoly positions of power states found themselves in after WW II. If a neo-liberal push toward privatisation marked the end of the modern era then this surely made a mark on discourses informing representations of ICW control and ownership. If up to this point com- 
plete privatisation of ICW had been limited by anything it would have been the amount of influence governments had in the institutional legitimacy. The neo-liberal push following world war two in many parts of the world would have been the last transition in terms of the governments role of distributing power to individual entities in the form of rights and privileged to being little more than an overseer.

The situation in socialist states such as China and Russia was significantly different however both adopting various forms of title and indirect reward for innovators and intellectuals through most of the 20th century. Recently China has colluded towards an IP regime following the rest of the world, while Russia occupies a similar position. Both, however, face very having a differing set of social representations around ICW ownership and control practices, and with their late entry to the homogenisation process, instigated by globalisation, must content with differing disruptive social institutions which are far removed in practise from the normative institutional frameworks provided by traditionally capitalist economies ${ }^{6}$.

\subsection{The advent of digital media and the post modern age}

Out of all the technological advancements which may have impacted on the social representation of ICW, none not even the printing press is as disruptive as digital media. Not only did digital media remove the physical medium from the equation, it promised to remove the traditional role of publishers. When combined with communication networks digital media has allowed a greater level of access to ICW than ever experienced in human history.

In a few short years, digital media went from a niche of the technological savvy to an all pervasive and rudimentary part of almost all industry and society. It changed the way news was reported and broadcast, manuscripts compiled, documents read, monies watched, music acquired.

\footnotetext{
${ }^{6}$ For a review of Chinese approaches to Intellectual Property in comparative frames, see Guanhong and M. Rose
} 
The seemingly vast commons of digital media available at the press of button became something taken for granted, and existing outside the realm of institutional controls.

For the first few years this attitude was definitely persuasive, discourses surrounding the social nature of knowledge became common place, along with various other rhetoric, which created legitimising myths around the free trade of various forms of ICW. Eventually the popularity and persuasiveness of the technology meant that the user base grew, and as this happened, more ideologies joined the fray. Many of these ideologies were in conflict with the anarchistic and tolerant nature of the institution of early adopters. The clash in ideologies would eventually become something which would enter into public debate. The most visible of these conflicts were the Napster case which came to a media highpoint in 2000. The Napster case pitted the rights of the recording industry against the rights of Internet users. The "fuzziness" of that description is a reflection of how ill defined either side's argument was at the time. Suggestions that it was a clear cut case of copyright infringement on the part of the Internet users, is a cursory approach at best. The full story is somewhat more complicated. What was at stake in the Napster case was essentially which ideology garnered more support, the social nature of ICW or the natural property right of authors. Essentially the fight was lost before it had begun. Copyright laws, especially in the US under the newly introduced digital millennium copyright act, were more heavily weighted in favour of creators and holders of rights than ever before. Even under the sometimes blurry guise of digital media distribution, to which Napster's defence clung to, which the small company claimed it was not actually copying content only providing a service which enabled other users to find each others content and share it. While effectively technologies like Napster are content agnostic and do no infringement by themselves the propensity for them to become leveraged in ways which communities find useful means they come to represent ideologies.

Cases similar to the Napster example, that challenge the limits of interpretation, have been occurring more and more frequently. Napster and similar technologies, products and services, tend to challenge directly the 
dominant market economics of media distribution networks. The conflict between the two ideologies was worsened by the slow adoption by industry and insistence on blaming enabling technologies rather than utilising them. While Napster, and more recently Grokster and various other Peer to Peer (P2P) file sharing cases reflect the disharmony in current copyright systems, they have created a rather vigorous debate over fair use rights. At issue is what social representation is dominant regarding exchanges involving ICW. Given the huge number of people engaging in what is now in some nations a criminal offence, on a daily basis with a varying demographics, understanding the social representation of both ownership and associated rights, and control based positive rights, rather than negative rights which current copyright law and international treaties such as TRIPS seem to focus exclusively on may provide insight into ways which fair use can be adequately defined.

The bundle of rights given to authors and creators of copyrightable material has become so compartmentalised as a product of ever more fine combed definition of a potential market. This is a good indicator that the representation of these rights has become objectified not as property or rights, but as a 'capital good"7. This economic social representation is a product of the hyper commodification, present in many globalisation discourses. The legitimacy of this representation is only perpetuated more so by the existence of organisations such as WIPO, and a push towards, total knowledge management practises, whereby knowledge resources are "captured" so they might add value to an organisation.

The problem with these approaches is that the treatment afforded to exchange practises may not fit the construction of ICW resources as economic goods. This disparity if properly understood could help inform current debates revolving around ICW control and ownership.

\footnotetext{
${ }^{7}$ capital goods, in contrast to consumer goods, are goods used in the production of (physical) capital. Capital goods refer to real products that are utilized in the production of other products but are not incorporated into other products themselves.
} 


\subsection{Patents and Copyright in the Media}

To understand better some of the most prevalent themes present around Intellectual and Creative work, we must look towards the landscape of digital media and the decreased communicative distances as facilitated by miniaturisation and the ubiquity of ICT technologies in society. These two technological advances have created not only a number of new subcultures, but have facilitated and empowered existing groups as well as disrupted old distribution centric views of ideas and their expressions. While the treatment of the data-set from the Patent Act review submissions provides a finely focused set of discussion, it is devoid of discussion for the most part about many of the discourses pinned to other forms of Intellectual Property law, namely copyright, but also trade secrets, trademarks and designs. The differentiation imposed by the Legal definitions are highlighted in the discourse different groups have opined surrounding these distinctions. Every day I am bombarded with articles about some legal action which draws into question elements of the general theme of Control and Rights surrounding Intellectual and Creative works, and the nature of knowledge. The debate is echoed by multitudes of blogs, news sites, petitions, action groups, legal advisers, software developers, technology enthusiasts, research companies', industry spokespeople academics, and collectives which tout varying degrees of support or disdain for the existing situation. Because of the much wider reach and scope of discussion in all of these forums there exists a much richer corpus, but potentially one without a single common thread.

Going back to the original intention of this thesis, which was to demonstrate the social representations surrounding how Intellectual and Creative work, and in particular to observe how these representations might diverge from the reified legal political-economic nexus's treatment, Internet and media are perfect pickings for helping illustrate further some of the previously identified themes, as well as adding to these.

As such the corpus here is illustrative. Examples of comments and dialogues from a cross section of articles related to intellectual and creative work are presented along with their relevance and position within a thematic map from which social representations can be inferred. 


\subsection{Concluding remarks and the map of histor- ical representations}

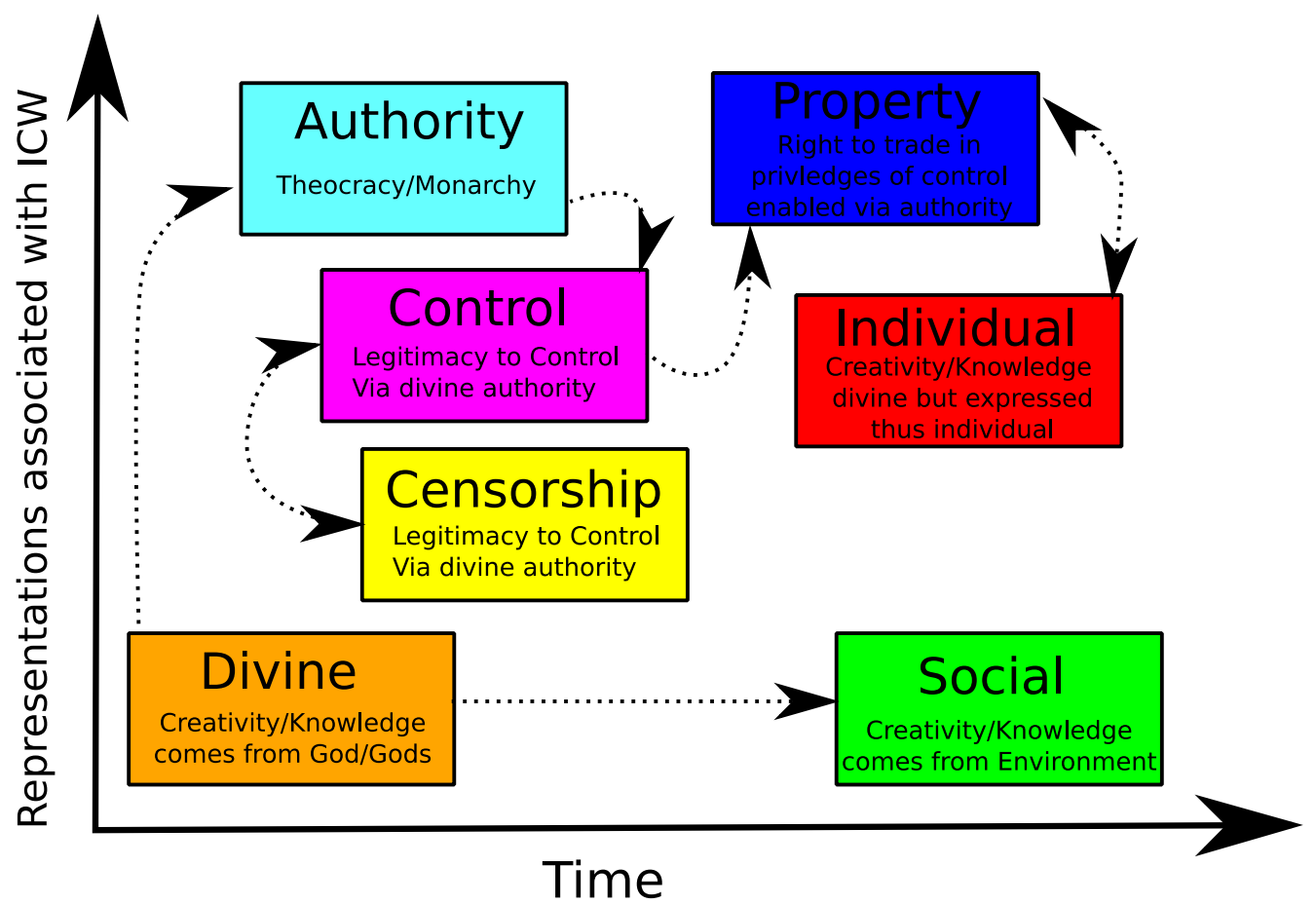

Figure 3.1: Themes present in social representations of Intellectual and Creative work and the nature of knowledge in societies up until the end of the 20th century (interactions between themes and representations are shown by arrows)

Omitted from this analysis is a closer examination of the various counter culture movements engaged in activities which, within the current ICW control environment, are subversive. The largest of these which has acted as a vehicle for increasing public awareness is the Free/Open source software movement. The Free/Open source software movement has championed the rediscovery of the social nature of knowledge representation and acted as a model for other disciplines, such as Biological sciences. Its particular expressions are somewhat unimportant, the fundamental persuasiveness of the movement lies in it's co-ordination and development processes. The distributed nature of knowledge resources are able to be 
effectively harnessed across spatial distance by multiple individuals. In terms of social representations of ICW, this is the biggest new discourse to be introduced by these counter-cultures. However for the purpose of framing current day discourses and movements, the purely narrative approach needs to stop here. Because current movements and changes are currently in what Wagner describes as a state of collective symbolic coping, any attempt to build a narrative around recent events needs to be detached from the historical treatment afforded to the topic of representations and themes of ICW in the past.

A synthesis of the various narratives presented in this chapter, in the form of a representational map (Figure 3.1found on page 83 )through time, presents a succinct summary of the narrative story I have built up around the literature and events relating to ICW. The use of a time component in the diagram is a novel component not following a strict reading of thematic analysis as presented in Braun and Clarke (2006). However, because of the hybrid nature of the methodologies used, and the increased explanatory power it enables by showing the various important interactions between representations over time (as represented by the arrows, double sided meaning both representations had significant influence on each other over time), it provides a useful basis to expand on with a more methodologically grounded study, to be introduced in the next chapter. 


\section{Chapter 4}

\section{The New Zealand Patent Act review}

\footnotetext{
"Intelligence rapidly creates satisfying, sometimes surprising plans that meet an array of constraints. The products of intelligence may be clever, ingenious, insightful or elegant. Sometimes, as in the case of Turing's solution to cracking the Enigma code, an intelligent solution exhibits all of these qualities. Modest tricks may accidentally produce an intelligent answer from time to time, but a true intelligent process that reliably creates intelligent solutions inherently goes beyond a mere recipe. Clearly, no simple formula can emulate the most powerful phenomenon in the Universe: the complex and mysterious process of intelligence.

Actually, that's wrong. All that is needed to solve a surprisingly wide range of intelligent problems is exactly this: simple methods combined with heavy does of computation, and an example of the problem. In some cases, we don't even need the latter; just one well-defined statement of the problem will do. " - The Age of Spiritual Machines: when computers exceed human intelligence Kurzweil (2000, pg 73)
}

Understanding the legal context that frames the legislative process, and the rhetoric in which the submissions to the patent act review are grounded, is important as it can be considered a reified world within an SRT conception. This introductory section describes those concepts, processes and terms, broadly considering these as Legal Thema that form (in part) a reified legal world surrounding intellectual and creative work. In this process of observing the reified thema, alternative common sense representations that incorporate legal thema are proposed so as to provide a second rhetorical frame for examining the themes from the data set. In this way the introduction section to this study combines elements of a analysis and traditional discussion section to allow more agility when discussing topics and themes observed from the public submissions. 


\section{1 "Intellectual Property" as Legal Thema}

Intellectual Property law is a catch-all term to describe a set of legal concepts and related policy and laws surrounding a set of four or more distinct types of law, each of which must be approached separately. They impose different sets of rights and restrictions on a society, and convey different sorts of exclusivity rights to the rights holder. Typically included under the Intellectual Property (IP) banner are - copyright, trademarks, patents and trade secrets. Figure 4.1 shows the distinct legal concepts and laws which are commonly and collectively referred to as Intellectual Property.

Outside of a legal context, Intellectual Property is commonly used to describe quite distinct concepts and objects which have little or nothing to do with the four legal principles, including knowledge capital, goodwill, and intangibles among other market based measures. As such, Intellectual Property considered from an informed legal-economic viewpoint, describes almost all forms of non-physical resource. As a phase space (the area of potential states in which a property of a system can occupy) it encompasses all human imagination and communication, the physical manifestations of the same, and the interactions within the environment and inhabitants. Various legal jurisdictions impose different treatments for elements out of a bisected segment of this phase space which can be considered human thought. Figure 4.1 shows these various treatments and legal fields as positioned using the idea/expression dichot separation paradigm 1

The underlying fabric upon which the legal distinctions are drawn is not well defined however, relying upon each type of law and legal system of control to operationalise exactly the parts of knowledge, creativity and express that are covered. There is no legal concept of the overall phase space itself, leaving each individual legally acknowledged segment; trademark, copyrights, patents, etc to overlap and converge in places. Because

\footnotetext{
${ }^{1}$ Table 4.1.4 shows similar separations and examples of types of Intellectual Property, as adapted from an official information sheet from an official New Zealand government website describing Intellectual Property http://www.med.govt.nz/templates/ MultipageDocumentPage 28277 . aspx
} 


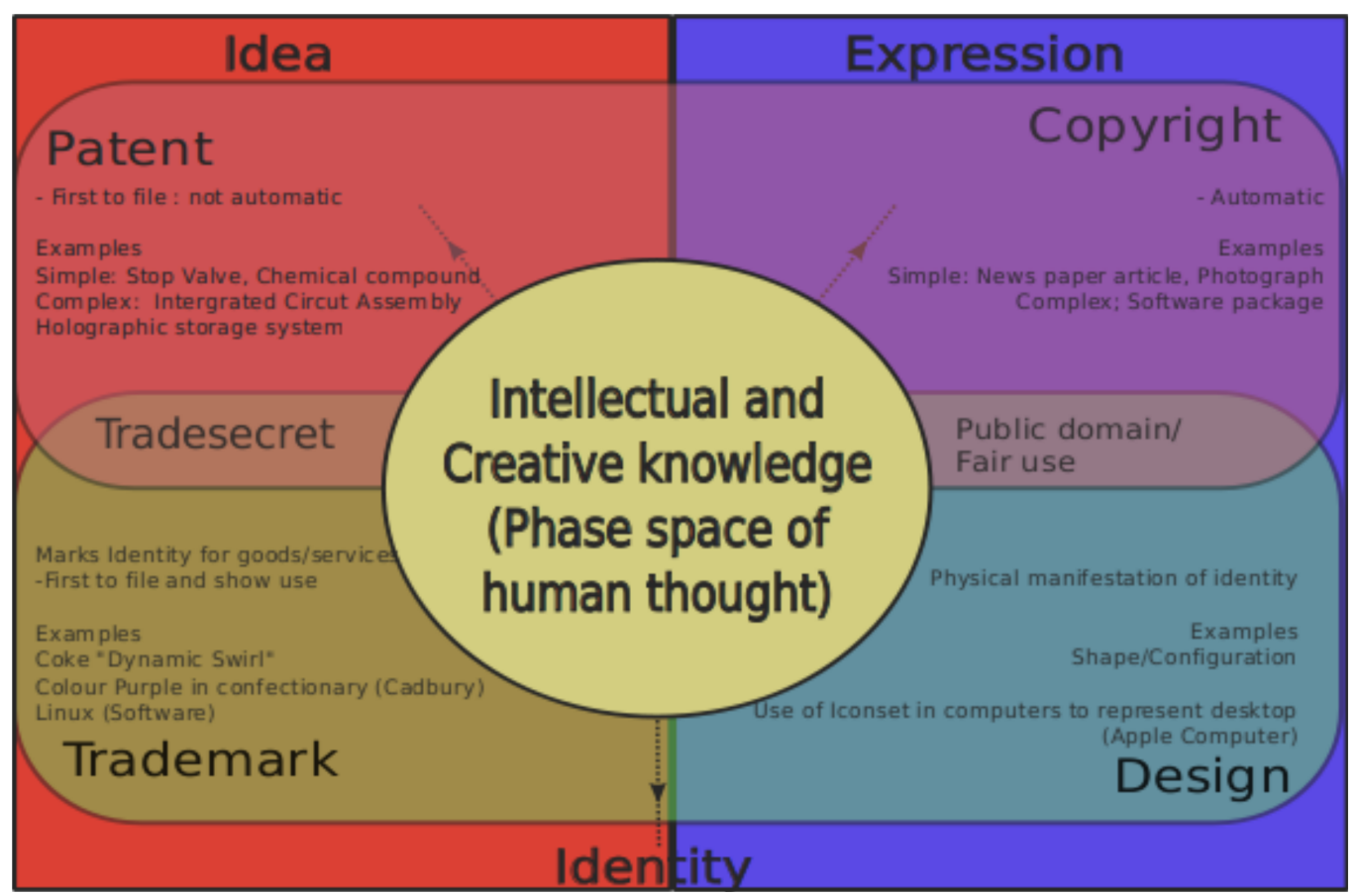

Figure 4.1: The legal fields which are collectively referred to as "Intellectual property" and the legally recognised and comodifiable types of segments of conceptual phase space/ ICW 
this thesis is more broadly interested in the underlying fabric upon which all of these legal distinctions sit, it is important to attempt to anchor define it so that the varying legal distinctions can be viewed together, because of the inherent commonality, as demonstrated by the umbrella term of intellectual property.

\subsubsection{A mathematical metaphor of thought and knowledge}

If we return to the Venn diagram, as consider it a as a phase space of thought. We can roughly mathematically conceptualise a function consisting of a set of potential inputs $i$, where $i$ is for simplicity sake based on the number of states in non-quantum physical reality. $i$ is dependant on the constant c, which is roughly the number of potential states the central nervous system of an organism o, can be in at any given moment $t$. Multiply this by the sum of all previous conceptual states an organism o has encountered in it's lifetime up to time $t$, and we roughly have the phase space of experience (and if we consider self aware organisms, thought, if we define thought as a collection of chemical-electrical states) for any particular organism o.

This function does not consider environment, which will undoubtedly constrain the set of inputs $i$ further but even if the input $i$ occurs only in environments e unable to support viable continued existence of the organism $\mathrm{o}$, it does not affect the phase space. If I were to teleport a badger into space orbiting a red dwarf star, the badgers nervous system is still likely to experience input $\mathrm{i}$ if only briefly, if even only to register how hot it is, and the feeling of being crushed as it implodes. The chances of this occurring are slim at best of course. Further when going back to a human as our creature of investigation, the conceptual phase space as a set does not make a distinction between inputs $i$ which are registered and those which are ignored or being variable in eliciting response (which could be considered for arguments sake as consciousness). For instance while my alarm clock wakes me on any normal given day, there are many occasions where this stimulus does not effect my rising (suspiciously correlated to the time I got to sleep, and how good the book/website I was reading was).

While we could plug some numbers into the equation, such as the av- 
erage number of neurons and nerves in a Human CNS (central nervous system), the average age of an individual human inhabitant on earth, and the number of possible combinations of states of energy and positions the organism/person could occupy in space, we would get still get a very very large number. Even when we throw away a huge chunk of the set by only including the likely environmental constraints it remains a very very big set.

An important aspect of this conceptual phase space is that it exists relative to time $t$. All potential states are transformed by all previous inputs and related states before time $t$. It is not something an organism chooses for the most part to engage, it is a fact of existing as a highly developed life form in a universe of whizzing buzzing energy. The problem of introducing any legal precedent which would give an exclusivity of sorts to elements of this phase space creates a problem of enforcement, essentially having to exclude other organisms which may inhabit a part of that conceptual space, and as such denies the reality in which all organisms exist.

This is a very low level mathematical conceptual metaphor demonstrating the difficulty in separating and defining at high levels knowledge and creative endeavour. And helps illustrate the hypothesis I want to present which is this difficulty in definition of ICW, from a societal and social level. That argument is simply that knowledge cannot be coherently treated as a commodity, at least not within a free-market economy paradigm. Any result to do so precludes the potential experience of others by having to operationalise a system of rights and controls around who is allowed to have access to a particular bit of knowledge, something which by virtue of the simplistic phase space illustration presented here, is inherently impossible unless isolating organisms from environments which they otherwise could inhabit. Like our badger experiencing the heat of a dwarf star, societies are being co-colluded into having to accept their environment as dictated by the conceptual spaces that are salient to them.

\subsubsection{Difficulties in the thema of Intellectual Property}

Unlike physical property or goods or even demarcation of labour - work, under which free market economics are based, there is no objective way 
to demarcate segments of the phase space without introducing properties that are not inherent to the system. Other clear differences between segments of the phase space and physical commodities, are that use of the phase space is not exclusionary in nature, that is, use of the resource does not prevent others from using it. Indeed use of the phase space only enhances it by virtue of expansion of an individual personal phase space through time. In essence any attempt to commodify the conceptual phase space into traditional economic models must do so through systems that must introduce artificial scarcity and constraints into the system. Technology has produced a system whereby access by individuals and groups to segments of the phase spaces in a relatively unadulterated form. Through "the network" a medium comprising of the Internet and communication devices and supporting technologies such as digital media and storage, the ubiquity of the network mimicking the conceptual phase space closely by providing near zero transaction and storage costs, and existing ignorant of the raw conceptual segments that inhabit it - information. The challenge again is how to impose over this medium the artificial scarcity and controls demanded of the economic treatment of the conceptual phase space, and this has not, until recently, been inherent in the technologies of the medium. This has led to a more natural evolution of behaviours and practises relating to conceptual space objects in societies in recent history because of a lack of interference from institutions. Those that benefit from maintaining scarcity through preserving economic treatments however have been put in a compromised position and have accordingly attempted to reaffirm through legal and economic positions the legitimacy and importance of this representation, as well introducing technologies that introduce controls and scarcity, to support the legal positions. The current legal system therefore puts control and therefore a commodified value of knowledge, into the hands of the few or individuals, when viewed globally, through these mechanisms. The situations seen in the past with the industrial revolution have many parallels now in the age of the knowledge revolution, we see greed in the media moguls, and research arms of multinationals as once were evidenced by the factory owners of the 1800s, and the same discontent at the situation from 
primary users of the medium, as from disgruntled factory workers. How both representations and uses are to coexist harmoniously remains to be seen, looking toward legal structures provides at least some grounding as to the common sense understanding of these themes.

There is some recognition of these differences between understanding the phase space as a commodity, as opposed to an organic shared conceptual set of experience and thought, existing in the set of Laws and premises surrounding intellectual property. This concession comes in the form of legally limited time periods of exclusivity over controls, which concedes the economic argument to that of a public good for a sacrifice in the short term. However this nod to the public is more and more ceremonial in practise. The admission that the entire system, and especially that of patents, is one of monopoly rights ( and thus exists outside of free-market economics to begin with ) is explicit in many discourses. The importance of these inclusions is that they inherently define a differing value outside that of economic conception, by implying the value that segments of the phase space have, may come from transmission and accessibility within societies. These are the reasons why limited periods of monopoly for most forms of intellectual property exist. Why a Patent (in most cases) is 20 years, why copyright is 50 years + life of the author (in many jurisdictions), however these provisions echo an era where distribution was controllable, where costs of transmission, duplication and organisation needed to obtain the monopoly rights in the first were prohibitive, either by complication of the legal process, distance, perceived value from investors or other constraints, most importantly that of a tie to physical goods, ink, paper, vinyl all of which enabled control over some aspect of distribution or manufacture and necessitated significant investment from some party. Today many of these factors do not influence the probability that a right may be applied for or granted in the first, essentially enclosing more and more of the conceptual phase space, off limits, via legal protections over exclusivity and control rights. Many recent reports and enquiries into the legal conferral of protections argue for increased global harmonisation, increased ease of issue for patents / trademarks, and copyright has been automatic in most jurisdictions for decades, but for further protections in 
this area of law also by highlighting the ubiquity of the network which makes inherent human behaviours more visible, including that of sharing segments of the conceptual phase space (Gowers, 2006; Drahos \& Smith, 1999; Yasuda \& Kato, 2007). Add to this automated on-line filing systems, a trend to make patent fillings as broad as possible, gaming of the patent system via use of sub-marine patents (whereby a patent holder files, and waits until a company using an element of the patent becomes profitable before seeking enforcement), increased complexity and the sheer number of patents now existing leading to backlogs of in examiners offices and poor quality of granted patents. There is plenty of evidence to suggest that IP laws should be relaxed, scaled down, and more rigorous and harder to apply for in the first, rather than the converse which fuels a growing publicly funded management and administration cost of maintaining current methods of deploying the patent system, both nationally and internationally. These two principles are at the heart of the conflicting positions that individuals orientate around knowingly or not, and the advantage as predicted by social dominance theory (Sidanius \& Pratto, 1999) goes to in power, status quo, groups. Acknowledgement of the public and individuals and in many cases organisations and groups which exist outside of the hegemony, even when they are the majority in numbers, is a central question for legal and policy decisions.

\subsubsection{Groups and trends in relation to legal thema}

Policy makers, industry body advisory groups and global organisations tend towards a positivist perspective where the ultimate goal is increasing global harmonisation, and streamlining the process by which applications are handled, as well as increasing the scope for which patents are applicable. Many groups wish to extend the period of coverage, and others seek to prevent research exclusions, others increased legal rights to create large portfolios of patents related to particular industry sectors, which allows creation of collusive groups, to prevent any viable competition by raising barriers to entry. These positions are strengthened by increasingly broad rulings in court for the types of activity and uses that relate to both copyrighted works and subject matter which is patentable. At least from 
a legal perspective the worlds political and legal powers are advocating for as much segmentation and exclusivity of the conceptual phase space as is administratively possible, creating a very narrow legal rule for how knowledge can and can't be treated. One which relies on the acceptance of a market-economics perspective of knowledge, and precludes at least in legal-reified worlds, novel and parallel treatments (Benkler, 2004).

The problem is that societies, cultures and people are built on a conflicting set of behaviours and beliefs about the conceptual phase space. People don't pick up a newspaper and think about how they are going to attribute how they have come across a piece of information, or that they legally should clear rights with the newspaper for using published interesting articles to pin up on the office news board. People simply integrate the information and use it according to the needs of the given setting. In this sense, knowledge is anchored to the social spaces people inhabit, the utility entirely dependant upon the interactions and environment that individual or group encounters. The right to make use of any given piece of information lies solely upon the ingenuity of the recipient (Benkler, 2000). With this construction of utility it is participation in an environment where the sources of information are explicitly made important, that determines how knowledge is treated ${ }^{2}$. While individuals and groups may decide that there is inherent value in a piece of knowledge, so much so that a market value should be attached, this is a product of participating in a market centric environment, rather than one of individual moral/rights imperatives. It is motivated by the psychopathic economic imperatives of many organisations, and motivation to control, based on an expectation that outputs be rewarded with monetary compensation and right of exclusionary controls, guided by first claim principles, in that the first individual or group to demonstrate they came up with an idea first is awarded exclusionary rights. Unfortunately even those who subscribe to this treatment also continue to participate actively in the free conceptual world, and also unfortunately for those same people, there are groups and organisations which operationalise different/non market value treatments for the same

\footnotetext{
${ }^{2}$ Academia being one where the importance of source citation is relevant for peer review ideals. Similarly in legal cases citing precedent is paramount
} 
knowledge that has come to be commodified under a market-economics social representation and valued by others. Some groups even extend their operationalisations of the knowledge and creativity to incorporate actively pursuing ways to ensure that ideas and expressions remain available to all. This can be explained either by nature of these groups inhabiting social spaces where the social utility of making available knowledge is high (file traders, for example could be considered to inhabit a social space where the utility of having a particular film is high, due to the want of others to see it ), or through an explicit understanding of the arguments for free knowledge. There are many other potential reasons and situations where groups choose to ignore imposed market centric legal suppositions, including for financial gain, but for the most part it is simply due to people living primarily in a free conceptual world, and for the expectation that Intellectual and Creative works and knowledge, indeed all segments of the conceptual phase space set, are a natural phenomenon and inherently free in nature. As demonstrated mathematically above by construction of a set encompassing the elements representing capacity for thought and experience, it is a rational and fair position to assume. Taken from this baseline position, any legal or social structures to regulate knowledge must inherently focus on attempting to regulate the environment a particular organism, or set of organisms is able to inhabit ${ }^{3}$. Because knowledge, and indeed any input which elicits knowledge, cannot be avoided nor can it be undone.

\subsubsection{Revisiting legal reified definitions}

Within the legal system this is defined in a number of ways, for copyright it exists as an Idea/Expression (Vaidhyanathan, 2001; M. Rose, 2002a) separation. This dichotomy separates the knowledge itself from a particular representation or going back to our mathematical model, input. Under

\footnotetext{
${ }^{3}$ Such as a farmer limits where his livestock can roam with fences to prevent them from encountering paddocks with toxic weeds, thus limiting the organisms environment, particular thoughts can only be regulated by preventing all possible avenues which an organism might encounter stimulus, you can't "take back" something once you have thought it
} 
copyright the particular set of stimuli the input, is assigned some sort of artificially exclusionary rights to limit the environments in which the stimuli appears. But the copy 'right' as legally defined, accepts that the knowledge itself the 'ideas' as elicited by the stimuli may be transferred, encountered or otherwise enter the environment of an organism through any other combination of stimuli other than the particular expression. A copy rights holder may exercise exclusivity rights to only that particular set of stimuli.

Table 4.1: Table showing various legal terms which are commonly referred to together as Intellectual Property

\begin{tabular}{|c|c|c|}
\hline Legal Term & Description & Example of Coverage \\
\hline Copyright & $\begin{array}{l}\text { Original literary, artistic } \\
\text { and musical works; perfor- } \\
\text { mances, sound recordings } \\
\text { and broadcasts }\end{array}$ & $\begin{array}{l}\text { Waiata, kapa haka per- } \\
\text { formances, koauau tunes, } \\
\text { weaving, carvings jewellery, } \\
\text { books, computer code }\end{array}$ \\
\hline Patents & New inventions & $\begin{array}{l}\text { New products, manufactur- } \\
\text { ing processes, new chemical } \\
\text { compounds, new technology }\end{array}$ \\
\hline Designs & Commercial designs & $\begin{array}{l}\text { Jewellery, fabric designs, or- } \\
\text { naments }\end{array}$ \\
\hline Trade Marks & $\begin{array}{l}\text { Brands, logos, names and } \\
\text { symbols }\end{array}$ & $\begin{array}{l}\text { Nike }^{\mathrm{TM}} \text {, Starbucks } \\
\text { colour purple on } \text { The } \\
\text { tionery (Cadbury) }\end{array}$ \\
\hline $\begin{array}{l}\text { Geographical } \\
\text { Indicators }\end{array}$ & Names of origin & Champagne, Feta \\
\hline $\begin{array}{l}\text { Plant variety } \\
\text { rights }\end{array}$ & New plant varieties & New varieties of apples \\
\hline
\end{tabular}

Patents, however, are concerned with the idea rather than the expression, in a patent paradigm the idea or knowledge itself is granted an artificial form of exclusionary power. The basis of a patent is that the knowledge the set of responses, regardless of the stimuli used to illicit it, are artificially constrained by the patent holders right as to what environments they appear in.

To illustrate further, let us consider the badger teleportation device, 
let's market it under the name, Badger Cannon, used earlier to transport our unwitting badger close to the furnace of our red dwarf star. If I choose only to Copyright my Badger Cannon, then if someone can figure out how my badger cannon works, then they are free to make similar devices based on the same principle (just not an exact replica) say for instance a Squirrel Cannon would probably be OK under copyright law.

If I however was to file for patent protection, other than reading the patent to determine how my device worked, no one other than the people I say, would be able to use that knowledge in any expression which relies on the same knowledge/principles as my badger cannon. Thus as patent controller I have complete control over the environments in which my knowledge can be applied to create stimulus.

Similar situations exist in terms of idea/expression dichotomy for what has been labelled as identity rights, which also warrant inclusion in discussion of the exclusivity rights in law granted to non-physical goods, and are generally included under the intellectual property banner. These are Trademark and Design rights. Trademark rights tend towards the Idea continuum, and design towards expression. Both however work on a different basis to patent and copyright, working off a principle of market value for enabling differentiation in a market, and thus are labelled as identity rights as they enable differentiation within a market. Trademarks grant exclusivity rights over symbols/shapes/logos and names as they relate to particular activities/goods or services. Unlike patents Trademarks must be shown to be actively being used/associated with the applied for categories of goods and services, and it is possible that if a trademark is not shown to be inherently related or continued to be related/used that it will be nullified. A trademark can also become so successfully, as to become associated and considered a commonly used generic label, which can also lead to nullification of certain exclusivity rights. A classic example of this is early 20th century company Hoover, which had registered its' name as a trademark, but because its' producing a line of vacuum cleaners that become popularised, leading to common usage of Hoovering to just cover the general activity of vacuuming it was effectively nullified, allowing other companies to market products using the Hoover as a verb 
in related products. Google is an example of a more recent but similar case, whereby Google has become a verb to describe general web search in general, than to apply to Google's search product(s) specifically, however Google has encouraged this practise. Also unlike patents, trademarks have an indefinite period of extension.

Design rights are more like copyright, but not just the entire expression but elements within the expression can have exclusivity rights applied to them. Such as curves and shape of a lounge suite. This is again on the basis that particular designs have inherent value in differentiating a product within a market. Design rights work more like copyright in practise, but are not automatic and must be applied for in a process that is similar to patents.

All of the discussion of the legal thema provides the tools needed to navigate the corpus of the current study, with the exception of a grounding within the specific process to which the Patent Act review itself is grounded, and the societal and cultural foundations which are may also be applicable to respondents and submitters within the corpus. The following section provides this grounding before detailing the data set and themes therein.

\subsection{The legislative history in New Zealand}

The first instance of anything resembling Patents within a specifically New Zealand legal construct goes back to the beginning of the last century where there was parliamentary discussion noted in the official parliamentary transcripts (referred to as HANSARD ${ }^{4}$ ) transcripts referencing developments overseas. However due to the colonial nature of New Zealand at that time there was very little discussion about patents law in New Zealand even around the introduction of the first parliamentary act specifically dealing with patents, the 1908 Patents, Designs, and Trade Marks Act. The current standing Patents act was read into law in 1953 and has

\footnotetext{
${ }^{4}$ On-line version of HANSARD debates are available for recent years, full historical copies are only available in hard copy from Archives New Zealand http://www .parliament.nz/en-NZ/PB/Debates/Debates/
} 
since been the principle legal mechanism guiding the New Zealand patent system. The original 1953 Act (a full copy of the act is included in the as appendix in the digital version of this document ) is approximately the same as the then British version of the same period. At that point, New Zealand was still being very much influenced by colonial legal and political allegiances to the commonwealth. Over the last 2 decades there have been a number of judicial decisions which have effectively amended certain aspects of the original bill, including branching of the legislation for particular industries, such as the Introduction of the Plants and Varieties Act 1987, which is itself implicated in a current review process. On top of this a number of legal cases have created precedent in New Zealand for certain applicability of the Patent Act to technological developments and industry not originally envisioned. In particular there have been exclusions; over Medical methods of treatment, and effective extensions to patentability i.e. A decision allowing Fisher and Paykel to patent algorithms stored in household appliance integrated circuits, Hughes Aircraft Corporation's patent applications - extending NZ law to cover software. As well as a number of other amendments clarifications and study notes that have been added over the years, as such the original Act has become rather unwieldy and is ill-equipped to deal with technological and social developments that have occurred since its conception.

\subsection{Specific New Zealand issues: Treaty of Wai- tangi}

One of the unique defining aspects of New Zealand cultural heritage is the Treaty of Waitangi which has for some time had legal and political power within the New Zealand state regulatory framework. During the 1970's following widespread collective social movements, the government would ultimately provide for recognition and legitimising of claims of historical grievances by Iwi (tribal) groups against the crown and private entities. The Waitangi tribunal was established in 1975 to oversee claims and settlements processes, and the importance and legitimacy of the founding document of the country were reaffirmed, and has since been inte- 
grated into fundamental government procedural and operational practises. The treaty itself sets out a number of obligations, concessions and commitments by both Maori and Pakeha groups to the governance of New Zealand (Aotearoa). Negotiated in the early formation of the country in 1840 and ratified by most leaders of Iwi groups existent at the time (with a few notable exceptions), it is one of the most detailed agreements to have been made between indigenous groups and colonial settlers in recorded world history, and one of the few instances where the British Crown entered into such agreements. The importance and interpretation of those agreements, which cover issues of guardianship, resources governance and the obligations to the same, in the face of technological and societal progression over the last century has had a defining role in New Zealand's political, legal and cultural distinctiveness (J. H. Liu \& Sibley, 2006; J. H. Liu et al., 1999). In terms of the treaty's influence and impact on how the Patents act and related legislation is formed, the original 1953 act makes no direct reference to the treaty, or treaty principles, having been enacted prior to the rediscovery and trend to incorporate treaty principle's into parliamentary legislation, which has been constant since the state owned enterprise act was read in law in 1986. The current draft act (included as an appendix in digital version of this document only) includes a component which seeks to address issues of Tikanga Māori and mātauranga Māori (Māori custom and knowledge). In terms of Treaty Claims which have impacted or have significant implication both in submissions, and NZ law, is the treaty claim known as WAI262 The indigenous flora and fauna and cultural intellectual property claim. This claim outlines a number of grievances against the Crown in terms of eco-diversity and cultural knowledge divestment and appropriation, both by the crown and by third parties authorised by the crown to do so. The outcome of the tribunal report is at the time of writing still in the final report preparation stage, after having gone through more than 17 years of hearings and investigations. Because of the broad scope of the claim which makes specific claims against current and past involvement in international treaties without Māori consultation (in particular TRIPS, Paris convention among others), and the western centric model of legal structures relating to issues 
of Mātauranga which have effectively led to exclusion, the outcome of the report will likely have major implications on the current draft patent act. It has been described in a briefing paper to the United Nations as

one of the most important claims of its' kind anywhere in the world - (Ross, 2005).

How these issues of traditional knowledge are incorporated into discourses and themes, and into the representational fields that compromise individual submitters and official channels is a unique deviation from many developed nations conceptions and implementation of the Patent legislation, and presents New Zealand a unique opportunity to exercise exemptions available in international treaties that recognise the place of traditional knowledge. It is also of interest for how Māori as a group are positioned in relation to submitters in terms of identity.

\subsection{The Data Set}

The data set consists of all of the public submissions to the Patent act review process, which commenced in 2002 seeking public submissions on what should be included in the draft act. There was a second round in 2004, which was more highly publicised in the media, but was technically designed to only deal with feedback about the released drafted Patent Act (released following round 1 submissions). However it sourced almost as many submissions as the original round. This second round included more submissions on policy issues concerning, and much feedback about, what should and should not be in the Act, as well as those submissions that were aimed more at dealing with technical wording and drafting issues. Many comments from second round submissions were either omitted from the round two draft exposure summary report/overview and combined media releases, or given a cursory mention. The Ministry justified this treatment in the overview documents released following the end of public submissions for the reason that:

None of the submissions identified any new issues that would warrant re-considering the policy decisions at this stage. 
The complete data-set used in this analysis comprises 65 submissions from both the initial 2002 round, and the 2004 second round. These were all the submissions that were available to be supplied from the Ministry of Economic Development, and released under the official information act. There are, however, notable omissions in this supplied set from the official submissions list that appears in summary documents, one of which is that of Zespri (a fruit and produce co-operative corporate body) which was withheld on the grounds that the submission would:

unreasonably prejudice the commercial position of the person who filed the submission

There are several other named individual submissions that appear to have been omitted from the received list that appear on the officially released lists, most likely due to clerical errors.

The official numbers from MED also may have separated some submissions where I have joined them in my analysis of the data-set (for example where two submissions are included as an appendix in official list ), and conversely joined some submissions where I have separated them. Appendices of Journal Articles, legislative errata, etc, were not included in the analysis, except during initial review of submissions or as references. In either case the number of submissions used in both remains the same at 65 even where there are some discrepancies in composition. A full list of submissions can be found in chapter 2 on page 44 .

The regulatory and competition branch also provided some level of classification in the official summary documents for each round. This classification has been largely ignored in the analysis as it is inconsistent over the two rounds, and the goal of the classification is unclear in both cases. ${ }^{5}$ It does provide a useful tool in comparison with my own classification system, and shows how the Ministry positions respondents in some cases. An example of this is where the ministry classifies individuals as Users of and Owners of both in regard of Patents and the Patent System in general.

\footnotetext{
${ }^{5}$ For example, in round one, the submission by Legal Firm AJ Parks is categorised as Legal practitioners, whereas in round two is classified as Patent Attorney firm. This becomes even more troublesome when considering industry and non-industry groups submissions
} 
Another example can be seen where the ministry discards identification of Business in favour of an Individual label for some submissions which explicitly state both and vice versa in other cases (such as for the submission 16 an ICT sector business with is positive overall in support of patents, which is categorised as business, while in another case where the respondent explicitly states they operate in a similar ICT sector, is categorised as unknown.

My categorisation treatment of submissions is based on originating primary activity as identified by the submitters themselves, with the core goal of attempting to keep nominal categorical groupings as minimal as possible without losing important granularity. Based on initial readings of submission I adopted four categories:

- Legal: This category included Law firm collective submissions, patent attorneys, and legal practitioners or Legal advisory bodies, i.e. Baldwins, AJ Park NZIPA

- Large industry and Industry groups: This category included publicly owned companies', large private enterprises and industry bodies that claimed association or advisory roles associated with those companies, i.e. Fisher \& Paykel, Fonterra, Researched Medicines Industry Association of New Zealand (RMI)

- Individuals and SMEs: This included the largest range of submitters and included individuals, small to medium size business owners, Trades people and Practitioners, engineers, academics, i.e. Management \& Technology Systems Ltd, David Shaw (a surgeon), Peter Andreae (an Academic)

- Non-Industry groups: This group included non-industry organisations, humanitarian groups, government bodies, Iwi organisations and political groups, i.e. Greenpeace, PHARMAC, Pacific Institute of Resource Management, GE Free New Zealand

Categorisation led to an extraction of the following nominal groups emerging from the data set: 15 of the submissions were categorised as Legal, 14 from Large Industry or Industry Groups, 21 from Individuals, small 
business owners or academics and the final 15 from non-industry organisations. The median length of submissions was just over three pages, however there are some substantially longer (with one submission of 160 pages not including an additional 90 pages of appendices) as well as a number of single page submissions. Some further length features present were those within a legal group tending towards the upper quartile, whereas, individuals grouping towards the bottom quartile.

\subsubsection{Thematic Extraction process: Tagging}

As discussed in the methodology section in chapter 2 the data set was subjected to a generalised thematic analysis (Braun \& Clarke, 2006) treatment as the main method of establishing themes present in the data-set. The main objective was to tag tracts of text with labels which were established from both a literature review of the topic and from initial reading and familiarisation with the corpus. The process involved establishing a set of labels which were attached to tracts of text taken from the submissions. This occurred early on in the submission process and re-readings and elaborations to the initial set of labels used were added as necessitated by the data-set. After this process had been completed submissions were re-read once again, and the broad range of themes extracted from the literature and historical review in chapter 3 (shown in table 2.2.1) were used to cluster tags and produce a thematic map out of the associated themes for just those which were applicable to the Patent Act public submissions data set. Keep in mind that the subject for this data-set is specific in its context in applying only to Patent law. However, as this is the most exclusive form of rights that can be granted over segments of the conceptual phase space it serves as a good basis for observing social representations of Knowledge and Intellectual and Creative work, with the goal of "snapshotting" current social representations and objects used to anchor these social representations of ICW in a modern day context, enabling elaboration on historical representations of ICW control as investigated in chapter 3.

It is appropriate to mention that detail and elaboration into policy aspects of tracts in the data-set, and/ or legal, drafting, legalistic principles or 
other issues that the public submission and legislative process was aimed at achieving, are not the goals of investigation. Rather the focus is on construction of underlying topics and themes, the social objects, used to navigate these issues. As such, while there are some parallels with government reports and analysis in places, the goals and thus treatment are quite divergent in purpose and scope.

\subsubsection{On overall Positive, Negative or integrated perspec- tives and consensus}

One of the first stages in the analysis of the corpus was to assign each submission a positive-negative valence label. This label was determined in terms of how, overall, the submission tended to agree or disagree with various status quo positions of increasing protections and scope. If the submission argued for increased or extended protections in some way that it was in support of a dominant legal-economic representation, and it was assigned a positive valence. A submission was assigned a negative rating if it disagreed with some aspect of the proposed and/or current treatment of a particular area of activity (i.e. plant varieties, software, business methods), or it argued for lessened protections in some manner, or appealed to explicit counter arguments. Submissions which offered a balanced and integrated perspective including aspects of both were given both positive and negative label. This rating system ignored most issues relating to legal wording/drafting issues, as they had little to no bearing on the overall position statement which the simple categorisation was designed to identify. However, it is interesting to note that submissions which dealt primarily with technical details overwhelmingly supported the dominant status-quo representation (that is a positivist harmonisation legal-economic position) of patents. Table 4.2 shows the distribution between the four different categories of submitter identified in the initial stage of analysis.

As can be seen from table 4.2, the split between entirely positive framing, and that of submissions which identified negative aspects is roughly even ( $\chi^{2}=0.02$ non significant). The categorical split also supports the notion that there is a definite dominant legal-economic pro-protectionist 
Table 4.2: Distribution of positive or negative positions of support for current or expanded patent protections

\begin{tabular}{lll}
\hline \hline Origin of Submission & Negative & Positive \\
\hline Legal & 2 & 12 \\
Large Industry or Industry Groups & 2 & 10 \\
Individuals,small business, academic & 16 & 8 \\
Non-industry group & 12 & 3 \\
\hline Totals & 32 & 33
\end{tabular}

theme, whilst the distinction between detractors is more varied. Overwhelmingly non-industry and special interest groups favour a reductionist theme. These themes, and the associated group, lend important weight and analytical power to further theme extraction.

\subsection{Extracted Themes}

A number of likely themes have already been suggested throughout the introduction by extracting representations and associated themes and anchors from historical perspectives and the cultural and legal discourses which have surrounded the development of Intellectual Property regimes in current day discourses. Table 4.5 as seen in the methodology chapter, shows a semi comprehensive list of these themes as previously encountered in literature reviews and historical accounts. It is repeated here for reference. The assumption of a legal-economic nexus and associated themes of economics, globalisation and property is hypothesised to represent the de facto legal reified view and as such when interpreting any submissions which do not question current systems for their conceptual validity, (and thus grouped as positivist in the initial coding) must be assumed to appeal to this dominant representation as demonstrated by the bias as discussed in the introduction of this chapter. This current implementation of Intellectual Property has been criticised as being ineffectual, and detrimental from societal, enforcement and education/innovation perspectives, and from a free-market and efficiency perspective by a range of individuals and groups in society (Boldrin \& Levine, 2008). Many of these criti- 
cisms are referenced by submitters in the data-set, as are arguments which support the current economic, innovative and legal benefits of the patents system. So as mentioned early in this section this conflict presents the core elements which differ in terms of how they are appealed to as baseline arguments both supporters and detractors, and are present within the submissions as expected.

We for example are a predominantly Pakeha group, yet we have objections to the privatisation and commercialisation of life forms and 'intellectual' property, and the theft of indigenous knowledge by those who wish to profit from it. The concept of 'common' ownership of resources for the good of all is deeply rooted in Pakeha cultural tradition.

There is also a conflict of interest for the NZ government because it is bound by the international TRIPs agreement which does not expressly allow parties to refuse the granting of a patent on cultural grounds. Multilateral and bilateral trade agreements could take precedence over domestic policies to the detriment of Maori.

It is this explicit invoking of various parts of the debate on the shortcomings, inadequacies and or the defence or appeal to current status-quo positions, as well as the examples used to support these positions within the submissions content on which the extraction of themes is focused. I use thematic analysis (Braun \& Clarke, 2006) to apply themes garnered from the historical review of literature as well as many un-cited references from general discussion in on-line forums and with peers over the years as a researcher interested in this topic. Where no theme exists and where there are sufficient observations from the data-set to support the creation of a new theme, it will be added as required.

\subsubsection{International Obligations}

The theme of international obligations is a powerful one, as it is used as a baseline supportive argument by proponents and antagonists of the current politico-legal-economic nexus. The appeals generally form around obligations to specific international agreements of which New Zealand is 
Table 4.3: Granular themes encountered in Dataset

\begin{tabular}{ccc}
\hline & Themes & \\
\hline Freedom of knowledge & Human right to communicative freedom & Economic Inefficency \\
Detrimental Effects & Incentivising Creativity/Inovation & Constraints on Creativity / Inovation \\
Coercion & National Mission & Global Mission \\
Natural Rights & Rewards & Public Good \\
Economic Rewards & Human Nature & Corporate Opportunism \\
Idigenous Rights & Trade Barriers & Harmonisation of Laws \\
International Obligations & Property & Business \\
Research & Developing Vs Developed Nations & Barriers to Entry \\
Education & Cummulative/Combinational Nature of Knowledge & Competitive Advantage \\
Individual Freedoms & Ease of Administration & Legal Precident \\
Business Models & Measurement of Value & Historical Validity \\
Censorship & Control & Power of Knowledge \\
Authors/Creator Rights & Non-exclusionary nature of information & Property \\
Theft & Fear, Uncertainty and Doubt & Technological Challenges \\
Dominance & Exclusions & Specialist Markets / Industry \\
Traditional Knowledge & Prosperity & The Public \\
Censorship & & \\
\hline
\end{tabular}

a signatory, in particular the TRIPs (trade related aspects of intellectual property rights) agreement. The Universal Declaration of Human Rights is another sometimes cited. Others include WIPO (world intellectual property organisation) discussion panel about Human Rights and Intellectual Property, and less notable international discussion panels and organisations are used as they relate to specific industries and issues. ${ }^{6}$ However international obligations are also used with non-specific reference to a legal/political treaties as a way of positioning New Zealand as a potential leader are used to support an argument where New Zealand has an obligation to demonstrate deviance from norms as an exemplary action for other nations. This is interesting as it communicates in a novel fashion that current norms and trends are inherently non-valuable or counter productive, both at a national and international level.

This extract from a private individual who makes no claim to be affiliated with any particular group demonstrates invoking the TRIPs as a way to support their position against inclusion of patentability of indigenous flora.

The NZ Government is bound by the international TRIPs agreement (trade related aspects of Intellectual Property), and therefore cultural-ethical exclusions to patentability could be opposed through

\footnotetext{
${ }^{6}$ For example 3 of the submissions which were primarily interested in the Patentability of plant varieties hold up an International Treaty on Plant variety and seed banks as an example of a multilateral agreement which should be honoured.
} 
the WTO....although a refuse may fit within the ordre public and morality exception... the basis for rejecting patents must be made explicit in national and international law... there may be a need to make exceptions to patentability for the 'public good'

The submitter here is demonstrating familiarity with an international treaty and using exemptions outlined in the document to support, using the processes outlined in the agreement, a loophole for a non-normative stance over a particular aspect of patentable knowledge, genetic resources in indigenous plants and animals.

In the following extract the submitter represents a non industry organisation, a government body which advises on pharmaceutical subsidies. They invoke international obligations in such a way to concede that difficulties in supporting a position of not engaging in a patent system would jeopardise New Zealand's international trade opportunities.

For New Zealand, costs of patents are likely to exceed benefits, as New Zealand is a net technology importer. However, to not award patents at all would involve risk and cost: it would make New Zealand vulnerable to diplomatic pressure and would result in the loss of benefits of being a signatory to TRIPs

Another part of the same submission includes a paragraph entitled Compliance with international law is important, the summary statement of which is

New Zealand therefore needs to accept some costs (buying into international law on patent protection) in order to gain the benefits of international law in other areas.

The same submitter goes on to conclude that we must honour the TRIPs only as much as is needed while making use of any and all means of excluding any areas possible while maintaining compliance.

Another extract which comes from an industry or industry representative co-op simply takes a differing viewpoint on international obligations where invoking the TRIPs agreement makes very little qualification as to meaning or ancillary motives. Therefore the remarks can be assumed to be in support of the status quo position. 
To ensure our international obligations are met where ever possible

And further along when discussing specific articles of the original 2002 discussion paper in regard to wording of New Zealand draft legislation

New Zealand's patent legislation should be amended to comply with Article27(1) of the TRIPS agreement to include the "capable of industrial application" (generally accepted as meaning "useful“) standard. Such an amendment would mean that an invention would have to meet the utility or usefulness standard before granting.

The use of international obligations is treated as non-contested within this submission, as such indicating an acceptance and positive attitude towards the status-quo position as outlined in the relevant documents (TRIPs).

A further quotation which uses implicitly international obligations as an incentivising mechanism comes from an extract from a Legal firm. Here the firm stresses the importance of International obligation via direct quotation from the trips and then leverages this by implicating that without transparency as provided in the TRIPs guidelines in implementing a patent system, social and economic welfare is at stake.

The "social and economic welfare" of New Zealand is and will be enhanced by the presence of a Patents Act that is considered adequate by both New Zealanders and foreign nationals. Where a patent system is understood and considered adequate by research based companies and investors, the likelihood of investment in New Zealand is increased

There are several sub themes which are linked to International Obligation, mostly associated with punishments both economic and developmental. The other is that international obligation is a baseline argument, that is present even in critical discourses of the current system. Thus International Obligation is ultimately linked to the theme of coercion and cooperation as well as harmonisation, and so is seen purely as a utilitarian argument against these factors. That this theme is so central to most submissions is indicative of the decreased distance between trading/social groups in the modern era. Up until very recently in history the 
idea of multilateral global protections were distant in thematic fields of social objects associated with ICW and control. While some global multilateral agreements have existed since late 1800's ( i.e the Berne convention) when considered globally only a handful of nation states had ratified them. The centrality here shows that most submitters conceive of protections now on a global basis, particularity because of the close thematic ties with economic representations of ICW. Global competition and global market incentives adds to its importance. There has also been a strong institutional trend since the 1990s with the steadily increasing emphasis on global trade issues being linked to bodies which emphasise commodification conceptions of ICW such as WIPO. This strong media and policy influence coming from the WIPO and WTO has obviously been effective enough to permeate social objects so as to incorporate the importance of International obligations and harmonisation, as demonstrated here. It could also be contended that a side effect of the push towards recognising international treaty obligations has been an increased emphasis on other non-intellectual property specific international obligations such as human rights, and as such multilateral agreements on human rights have also been incorporated into social objects associated with ICW within recent discourses ${ }^{7}$.

This International obligations theme leads us to the next major theme which is also universal, and through which the international obligations theme mobilises as the metric to which it claims importance, that is of market economics, business and trade.

\subsubsection{Business, Economics and Prosperity}

The theme of business had emerged from the roots laid down in history as censorship and royal favour, and became central in ICW representational fields after the introduction of the printing press in Europe led to publishing guild members swapping royal rights to publish amongst themselves. Eventually this would lead to a property treatment of and a market for trading in the property of rights. Individuals soon joined the mix and

\footnotetext{
${ }^{7}$ examples of this from the data set can be seen in later in this chapter under the rights theme section
} 
eventually royal favour gave way to private rights exchanges. This property basis has become heavily connected with the dominant representational field of patents. Within the data-set an overwhelming number of submissions made appeals to how the patent systems should be tailored to produce the best environment for business acumen to prevail. However arguments both for and against the increased protection afforded by the patent system are also distributed almost evenly among the submissions. The conflict between relaxed or increased protection arises primarily from arguments for whether exclusions should exist for a particular domain of human endeavour, and also on raising the technical standard for which novelty is considered. This emphasis on exclusionary activities exemplifies that when appealing to business sense, there is a reliance of self-evident cause for creating an optimal economic and business operating system. This is a strong theme in the overall representation of ICW that counters protectionist arguments strongly present evidence of continued economic prosperity/opportunity, more so than proponents. Proponents of increased protections are noted to generally state that the current model is good, more protections are better for them and economically. Similarly arguments for decreasing scope/protections are positioned in the same way economic loss is emphasised, generally through a linking to competitive advantage positioning. To use a psycholinguistic term the commodification of knowledge is given an almost baseline argument status in discourses (Billig, 1996) when business themes/repertoires are invoked. When business themes are involved the commodification/economic value conception of knowledge is presumed as a de facto- requirement for business to operate and for economic success, which is can be considered part of a good/bad thema dichotomy. This is evidenced by the complete lack of acknowledgement of alternative conceptions of societal value/operating models in almost all legal submissions and in submissions where alternatives to a ICW protectionist business models and operations are given they are associated with negative effects, but see Perelman (2003) for alternatives.

The following are submissions from a legal firm, from round 1 of the public submissions with particular reference to the issue of software and 
business method patents as raised in the official MED document asking for public submissions on a set of issues that had been identified in the 1953 Patent Act bill.

The firm campaigned for many years to have patents granted for software inventions and successfully represented the applications in Clarks Applications (1993) and Hughes Aircraft Company's Applications (1995), two cases involving software inventions where the hearing officers allowed patents to be granted.

The submitter identifies stake, and that they in particular had been successful in the past in securing legitimisation of their interests (or those they represent being a legal firm). In this they embed themselves within the status quo and as having been instrumental in establishing the status quo and having it ratified in the past.

In discussing developments and responses to a European consultation process on business and software patents the author presents the options identified in the consultation document; harmonisation, more restrictive approach, more liberal approach ( as in the US at the time) - The submitter summarises the submissions as such

Those favouring a liberal approach comprise lawyers, established industry players and government agencies. Their concerns for the continuance of software patents are the protection of development investment, equality with the US, and an opening up of global markets. Their proposals include the application of current European Patent Office practise in the National; offices of Europe, applying patentability criteria to software that are slightly more liberal than those proposed in the Commission consultation paper, and to take extreme care with patenting business methods.

Those taking a restrictive approach comprise students, academics, engineers and start-up companies. They see software patents as threats to the open source movement and small to medium enterprises (SMEs). Their concerns include lack of patenting resources and expertise, fear of litigation, negative impact on standards for interoperability. Their desired proposals are to severally restrict the patentability of software, limit infringement liability for open source software, and reject all business method patents 
In the submitters summary we see two distinct groups constructed. In line with the predefined stake of the submitter they sympathise with the group is labelled as liberal that the submitter includes lawyers in. The submitter goes on to justify this support with the following statement.

The group favouring a restrictive approach numerically dominated the response and a large proportion of this group was explicitly from the open source movement, including the Euro Linux petition. However, the organisations with the most economic weight favour the liberal approach and are included in the included in the industry and other associations. On this basis, it can be argued that there is an economic majority ${ }^{8}$ in favour of patents on computer-implemented inventions.

The submitter constructs a number of important themes in this tract which have significance to this study. The first is the acknowledgement of a majority position against their own, via use of a disclaimer (Potter \& Wetherell, 1987) which is used in this context to deny the traditional construction and define a new criteria for measuring democratic majority. The criteria the submitter introduces is that of an economic majority as the qualifier, which is used to mark importance (Potter \& Wetherell, 1987). The second important aspect is that through use of further group demarcation the submitter highlights a particular group within their construction of restrictive position group to hide potentially more legitimate associated groups (such as academics, engineers and start-ups, as well as lawyers, governments and industry groups which do not conform to their position). This is accomplished through the use of discriminatory language to position this particular group as being incompetent.

The many anti-software patent respondents who are clearly unaware $^{9}$ that there were already over 20,000 software-related patents in Europe should be informed about potential benefits of patents and the procedures for applications

The economic theme appears to have two distinct features which appear to be tied to differing representations of the property social object. In

\footnotetext{
${ }^{8}$ emphasis added

${ }^{9}$ emphasis added
} 
the hegemonic/dominant social object comprising of the themes of economics/prosperity and business there is a reliance on the property as a physical good, i.e. treating it as having all the exclusions that a physical resource is subject to. This is the representation that is mobilised by legal and large industry organisations, and from an economics theory perspective most resembles simplistic classical economic models. The second representation removes the reliance on a physical good anchoring of property, and so adjusts the economic models and associated themes of business and prosperity to include more intricate versions of free-market economics. This argument is quick to highlight deficiencies with the monopoly exclusivity created by patent systems, as well as the innovative drain they can have on their modified business themes. Both however share the commonality of economic prosperity as being good, however they mobilise the social objects differently. The reliance of the dominant position on what appears to be a physical resource anchoring of property appears to be key in how subsequent arguments and positions are framed so let us examine that theme next.

\subsubsection{Property, Exclusions and Control}

Many submissions invoke the property metaphor of intellectual and creative work, either explicitly, or implicitly. Because the property is metaphor linked to most recent legal conceptions and more recently within the media, it is the single most prevalent representational symbol transmitted at an institutional level. While many submissions attack the property metaphor by introducing arguments for exclusions, and thus go on to define that many of the ideas and creativity involved with certain types of activity, such as biotechnology, software and business methods, do not constitute property because of reasons of intuitive, or inherentness, rather than innovative process. This dichotomy of intuitive versus innovative appears often and is specially placed in the property discourse because, while itself drawing from a legitimacy theme as produced via the institutional messages and symbols entwined with neo-liberal property discourses. It constructs property, as being a valid treatment of knowledge when it has the legal backing confirmed via the patent system, rather than 
being an inherent characteristic. This distinction however is often overlooked in rhetoric, and could be considered as evidence of how influential the property metaphor has become when considering exclusivity rights. The following extract is typical of themes of anti-property social representations present on the Internet. While it is not part of the patent submission data set the anecdote offers insight into how certain groups tend to disagree and dissociate property themes from diversified social objects of knowledge, where in dominant representations the property aspect is highlighted. Property themes are not as actively debated within the patent submissions as expected, with only a few submissions drawing into direct question the concept of property. It is possible that the distinction between patent and copyright is such that patent topics do not illicit the same disassociation from property ( especially physical property anchors) that copyright debates seem to because of the need to apply for patent. Copyright exists de facto upon expression in most jurisdiction, and thus groups have more direct encounters with copyright in day to day life, whereas patents are hidden from view, and not subject to individual experience in obtaining and dealing with them, regardless of the fact that many house hold items, within the end user agreements (usually found in product manuals in fine print) include clauses relating to patents and particular uses.

This extract comes from a threaded discussion forum on the popular technology and culture news aggregation site slashdot.org. ${ }^{10}$

Copyright is intrinsically immature. Copyright is claiming ownership over other people's physical property based on its similarity to your own (only the physical exists, only copies of information exist, a "work" in the abstract doesn't exist) It's much like a toddler's attitude on a larger, more effective, scale.

I say if they don't want stuff copied, then don't release it - fine by me. The opposite to copyright is demanding that artists release their work and pay us to listen to it, not no copyright (copyright: a right to restrict the distribution of copies of already-released copies

\footnotetext{
${ }^{10}$ The full discussion thread can be found at http://news.slashdot.org/news/ $08 / 04 / 27 / 2226237$. shtml. The thread's originating article is about the exclusion of a noted copyright expert being un-invited from a symposium on copyright being held in Canada.
} 
of information). I'm in the "no copyright" camp, not the oppositeto-copyright camp - I would oppose both copyright and oppositeto-copyright, supporting only physical property rights in the media that underlie information patterns. They're the only thing that even exists!

From within the submission corpus we find it is more common that submitter makes a plea towards the social nature of the representation of knowledge, or in some cases, particularly in those involving biotechnology and traditional knowledge, towards the representation of knowledge as being sacred ( consistent with the historical representations observed in chapter 3 ). The groups which make these challenges towards the property metaphor are important, among them include software developers, biotechnologists, geneticists, and cultural heritage groups, such as Iwi (tribal Māori) organisations, and government industry watchdogs like Pharmac. The striking feature of all these groups is that they are weighted towards high-technology areas, such as IT, and bio tech, or conversely on the opposite end of the spectrum in a humanitarian/not for profit area. Many of the individuals which comprise this nominal hightech group can claim membership to larger business organisations in terms of the creative and or engineering endeavours they may carry out. Larger organisations do tend to support increased protections to the exclusivity rights, by reaffirming the property metaphor. Individual innovators, researchers, independents and engineers tend towards a directed disruption of the property metaphor either by supporting a number of positions which emphasise technical and/or operational aspects of their respective trades, or in the case of traditional knowledge towards a basic human rights approach to knowledge. Potentially this is because they benefit from having an 'open access' culture, and recognise the need for freedom of expression and increased risk of continuing their behavioural and creative enterprises in a heavily regulated, first to file/buy/licence operating environment that a mandatory patenting environment necessitates. In these situations the patent system inherently favours large well established organisations and states (Boldrin \& Levine, 2008; Nachbar, 2006; Allred \& Park, 2007; Warner, 1999). However, the myth of the cowboy inventor is 
promulgated by the legal system, lawyers and is the modus operandi under which many incubators, venture capitalists and start-ups assume. The majority of patents awarded go to large corporates, and smaller organisations tend only file to avoid litigation in many cases, or so they can be swallowed in one time pay outs by larger organisations seeking to increase patent portfolios or as part of settlements. This does not lead to innovation but monopoly over arbitrary segments of the free market, as facilitated by patent pooling (gathering all patents by a single organisation around a particular technology/industry, so to exclude/charge monopoly rents on potential competition).

This is a situation which the software industry is well aware of, and parallels exist in genetics and pharmaceuticals (Kapczynski, Chaifetz, Katz, \& Benkler, 2005; Heller \& Eisenberg, 1998) and has existed in the past in areas such as automotive and engine mechanics (Boldrin \& Levine, 2008) radio aviation, and many other segments (Vaidhyanathan, 2001). The resultant monopolies have led to huge monopoly rents being charged and an overall drain in both innovation and funding into the $R \& D$ (research and development) sector in the past. On the surface from an economic perspective the increased funding and development investment looks to be a good economic indicator, as is touted as such (Gowers, 2006; Yasuda \& Kato, 2007). But doubts have been raised over whether the increases in investment especially in R\&D is due to the inefficiencies in the patent system, and that companies are being forced to research around existing patents rather than enter into licensing pacts at monopoly rents (Benkler, 2002). The earliest example of this sort of market segment monopoly being enforced through patent pooling was the steam engine, to which James Watt held several patents over and bought the rights to others which formed integral parts of the workings thereof. Other inventors significantly improved upon his design early during its' introduction but were barred from constructing/manufacturing their designs for more than 15 years after the invention, despite the radically more efficient designs, because of the market control Watt held over elements integral to basic elements of engine design.

Returning to themes around the limitation of what can be constituted 
as property, we encounter themes which imply a reliance on a social nature of knowledge conception heavily. The following tract coming from an Individual/consultant working in ICT sector.

I am heartened by your summary of the present patent system and its rationale. I am heartened that you recognise that patents granted for non-genuine innovations only cause net cost to society.

Please also recognise that the patenting of even truly innovative mathematical algorithms has never gained legislative approval. Advances in mathematical science have always been freely available for public use. It is a deductive fallacy that just because you can express an innovative mathematical algorithm using a computer to achieve a commercially useful effect that you should be able to get a monopoly upon that innovation.

Endeavours such as software, multimedia, advertising and general research seem to challenge to the conception of code/ideas/media as being regulated by law of exclusive rights rather than creativity and utility. This is demonstrated by a representation which emphasises difficulty with the property metaphor and its' highlighted feature of individual exclusive rights. This is supported by similar rhetoric and reluctance to use a property metaphor in on-line communities in chapter 3. One suggestion is that because of the ease at which information, now ever more diverse with the prevalence of adoption of digital media across all industries, is shared via ICT networks. The amount of available information in various myriads of forms, and the ease of obtaining diverse information, without transaction costs, from this immense pool makes it difficult to navigate using an exclusive rights social object. One of the fundamental aspects of a social object is that it allows groups that utilise it to navigate both new information, and environment successfully, (Wagner et al., 1999). Hence, the problem with a property metaphor is that it lacks any useful redeeming features in a landscape where accessing and utilising information does not impart significant benefits on the receiver, and few if any costs on the transmitter ( and in many cases benefits the transmitter, reversing the typical conception of exchanges when physical goods are involved $)^{11}$

\footnotetext{
${ }^{11}$ An example of this, is that example where Music sales are increased via giving
} 
The conflict in conceptions of how typical economic exchanges occur underlies this theme. To many groups there is value, both in terms of the ease of which they can navigate this information landscape in terms of a social object, but also in terms of the value of having the ease of access and the increased diversity that it potentially can add to their own creative and intellectual endeavours, and so discard the property metaphor. However groups which primarily rely on the property metaphor to engage their worlds, tend to do so with a the property metaphor being embedded within their social object of economic prosperity, and so defend the property conception of intellectual and creative work as a baseline argument to sustain their economic social objects. Groups that are observed to challenge or present difficulty with a property metaphor have expanded social representations of economic prosperity and as such are more willing to incorporate new information into that representational field.

These issues are not entirely new and have appeared several times in history, notably following the printing press' introduction and subsequent widespread adoption, which also enabled the collection and access to knowledge on a scale unimagined before. Unsurprisingly the philosophical debate arising out of increasing recognition afforded by the public to individuals and authors involved in the production of creative works led to the first occurrence of the property metaphor being conceived. However the key mediator in sustaining a monopoly rights framework based on the property metaphor, has been the distribution network, as provided by publishing houses and centralised repositories. This step is the very thing removed by the current ICT environment, so the property theme has once again become challenged. While traditional manufacturing has been used during the last half of the 50th century, to tie the property metaphor

away/making available freely via Peer2peer networks, a new album/movie/TV-series. While there are many free-riders these are not considered a market, as they would never have purchased the album had it been protected by some means (Digital restrictions management etc) anyway. However the increased exposure in a diversified market place created by allowing free-riders to effectively increase both reputation, exposure and fan bases, allows for new market value to be captured from the knock-on of the increased exposure) This however ultimately distributes cash flows, which has meant reductions in revenues in traditional markets which may not capture the diversified market as effectively 
of creative and intellectual works to physical goods, such as CDs', Prefabricated design solutions (Lockwood homes), paintings and DVDs, anything where expressions were inextricably linked to physical format. Digital media has removed this physical element making the metaphor more tenuous to maintain. Unsurprisingly it has been the purely creative sectors which have first discarded the property metaphor, most of which have few ties to physical manufacturing processes. Software development and engineers that work with the network being the first to re-envision the social object of property into something more aligned with the environment they work with (Lessig, 2001), and followed by other creative industries such as musicians, artists and finally businesses as the network metaphor and digital media has spread to every facet of society. While there are many businesses now that have adapted or grown out of the operating environment created by the network, and have adjusted their conceptions of intellectual and creative work as something beyond physical property metaphors, they however are still a minority and as traditional businesses still rely on traditional exclusive rights physical property metaphor as anchors for their operating and strategic directions, the social object of ICW is still associated with classical economic representational fields. Because the re-represented property metaphor that removes physical resource mappings, disrupts current dominant groups that have achieved their position largely through traditional economic practises, the threat of destabilisation causes increased efforts by dominant groups to maintain or strengthen the system to continue to favour them (this could be considered a classical example of Social Dominance Theory in action (Sidanius \& Pratto, 1999))

\subsubsection{Human Rights, Traditional Knowledge and The Pub- lic}

Human rights appear as both a naive opinion and as developed arguments stemming from a wide reading of both Intellectual Property Policy and counter arguments. The crux of this theme is based on a basic need human right of freedom of thought. Human rights are invoked in a number of different ways, which can be thought as broad ranging arguments in 
favour of a basic human right to creative expression, communication and to be free of persecution for being the recipient of controlled knowledge. These are logical extensions anchored to earlier human rights such as the right of free passage, journalistic rights religious freedom among others. There is a developing sector which considers that under current politicolegal frameworks set by Intellectual Property, these are challenged basic freedoms which need to be institutionally protected. The following extract relates to the human rights themes relating to property, and sustainable development, and comes from a non-industry group submission and is elaborating on a United Nations human rights commission statement about intellectual property rights.

In the unprecedented resolution, the UN sub-commission for the protection and Promotion of Human Rights pointed out the dire consequences on the human rights to food, health and self-determination if the TRIPS agreement is implemented in its current form. Reminding governments of the primacy of human rights obligations over economic policies and programs, the resolution states that there are " apparent conflicts between the intellectual property rights regime embodied in the TRIPS agreement, on the one hand, and international human rights law, on the other"

Examples relating to how IP rights have been used to stifle self determination and freedom of expression have become increasingly common, with companies using a wide range of IP rights to silence detracts through legal mechanisms. In one case, Apple Computer attempted (successfully in one instance) to silence blog writers over leaked product information using both monetary incentives and facets of copyright, trademark and other legal IP enforcement avenues. In these cases human rights of freedom of expression are generally cited for resistance. A corollary found in many debates in many countries, including New Zealand, is the right to re-use copyrighted material, which may have been legally obtained, in context outside the original conception. Many countries including that of New Zealand have no provisions in law to allow for transcoding, (moving from one format to another i.e. from CD to digital media files, like ogg vorbis or mp3) of copyrighted works to other formats, making common 
behaviours, in some cases criminal offences. Exemplifying the underlying human rights debate is that technology is being developed to allow for recognition of music/songs in public settings, and more generally what are called Trusted Computer and/or Digital Rights Management, making the enforcement of only a particular conceptual use possible. It is not far fetched that with advances in consumer technology that scenarios where humming a copyrighted song on the street could find you instantly charged for royalty fees. The challenge for human rights themes is to identify the boundaries and areas within existing legal structures which protect the rights of individuals, both in privacy contexts and civil liberties and the right to expression of opinions and freedoms. But more importantly and central to this theme, is the hard bias towards authors and rights holders as the de facto representation in the current legal reified world. This stance has led to inevitable conflicts and a debate surrounding the common human conception and treatment of knowledge and information in everyday lives of people. This has become more ubiquitous with the dawn of the current technological revolutions in distribution and group formation as facilitated by communications technologies such as the Internet. As Spini and Doise (2005) correctly identify, a right only tends to become salient, and in need of protection when it is threatened. Because of the relatively unchecked creative expression afforded to early adopters of the communications technologies, utilising the ease of distribution and edit-ability of digital media during the later part of the 1990's and early 2000's, Lawrence Lessig (Lessig, 2001) a legal theorist and lawyer who specialise in intellectual property law, has been instrumental in creating legitimising legal structures (such as creative commons licensing system). He sees a threat in the current legal and policy themes that are turning toward the network and digital media, going so far as to state that the current disruptive technologies are/have enabled for a more participatory society, that is now being threatened both by legal enforcements and technological means. The importance of the liberalisation of cognitive time is echoed by other theorists, who have made similar references to how automation has given societies huge amounts of cognitive free time since mid 20th century, but we have absorbed that additional free time in 
passive media such as television. The network as a participatory medium is starting to re-liberalise some of that cognitive surplus into meaningful artefacts, however legal systems are a threat to this liberalisation, and economic systems that have emerged over the last 20 years around IP are at odds with a massive influx of knowledge capital that could potentially be unlocked (Shirky, 2008).

The threat these two authors are referring to primarily is the legal underpinnings of copyright, and in particular the media cartels which traditionally occupy and controlled distribution channels during last century. But the main position is that copyright locks away creative content behind legal barriers, requiring complicated rights clearing before any part of any creators work can be re-used. The primary purpose behind the Creative Commons foundation essentially is to subvert copyright law to cope with the changes to sharing and distribution that digital media and the network creative landscape have facilitated. Creative commons offers no solution for patents law however, but the principle concepts behind creative commons, (which in essence uses contract/licensing law to circumvent parts of copyright, by default allowing for less restrictive allowed use of an artefact's) is reflected in the number times the human rights platform is raised in the submissions to the patent act review. As already discussed in other themes, the strength of protections for rights holders is difficult to confront head on, so successful arguments tend to be novel ways of circumventing these rights rather than purely confronting a particular representational structure such as property. Human rights themes are incorporated in a similar fashion in that even strong opponents of generalised themes of property and ownership over abstract knowledge structures, as assumed from associative group information obtained from literature and Internet and media study, tend to avoid presenting direct challenges but use indirect methods to challenge the increased protections position. The general progression of the development of the human rights theme is observed is as follows.

First a challenge or example to how the patent system either fails to protect standard behaviours and practises is identified.

The basic problem with these Reviews is that the government 
does not have the right to grant patents or proprietary rights over indigenous flora or fauna, or the products extracted from them...

Second a referent legal reified world is referenced, using the legitimising theme of obligations to justify a particular action.

... should be given by the government while the WAI 262 claim remains pending. By conducting these Reviews, the government is not only presenting itself as though these rights are the government's to give away or sell; but is putting itself in a position of restricting what the possible outcomes of the WAI 262 claim might be.

Third an action or step to rectify the situation is presented.

There must be a total moratorium on the granting of any patent or proprietary right over indigenous flora or fauna, or the products extracted from them, until there is resolution of the WAI 262 claim.

This sequence of dialogue is similar to the conversational/discourse analytic concept of trouble (Atkinson, Heritage, \& Maxwell, 1984). However given the asynchronous nature of the submissions, responses are expected to be reflected in changes or alteration to the draft patent act document. But it can be assumed that given the level of familiarity with both the legal process and the interest of participants being high enough to warrant a public submission in the first, that they have at least some awareness of other groups and their differing positions, as well as the dominant structures that exist in the sphere to know when a theme they are introducing may potentially be recognised as trouble, and therefore in need of clarification. It is the extensive clarification of human rights themes through use of references to authoritative sources, and international obligation ( i.e. Geneva convention, UN ) and common practises of associated groups which operationalise the introduced theme, that the human rights representational field is introduced within submissions.

The most important aspect in relation to the cluster of themes is that Human rights are now demonstrably integral to social objects that constitute the representational field of intellectual and creative work. While this social object tends to only be highly mobilised by watchdog groups, and is non-existent in Legal and Industry submissions, it is has a high referent legitimacy, appearing in a number of international and multilateral treaties. 
In terms of the value of this observation to the research goals of this thesis, this lends support to the formation of a new component of what, was identified as a Social Nature, social object of ICW in the historical analysis.

\subsubsection{Authority and Legitimacy}

Who has the authority to review patents, and to verify if an idea / patent application is worthy of receiving protection, forms another theme present within the submissions. It is one of the three themes acknowledged to be present, in the official overview of submissions released by the Ministry of Economic development in a media release ${ }^{12}$. The legitimacy theme takes central stage for a large swathe of legal firm submissions, because it influences and provides the mechanism for which they operate, hire and train their members. In the US it is customary for a business to employ/retain its own legal aide as a permanent staff member. Within the EU and New Zealand this is not a common occurrence. Many businesses especially those which operate in high-tech or creative industries which are attempting to work within the confines of the current hegemonic conception of knowledge are challenged to be equipped to deal with the patent application process, cross-licensing and various other checks which have become essential for those working within a traditional business model. It has become increasingly difficult for those companies to ensure that they are complying with the various laws, regulations and methods that are essential to the Intellectual Property regime currently in place, not only in terms of whom to engage in these search and application processes, but how to navigate increasingly complex licences which may prohibit certain uses of a product, or even be aware of a licensing requirement at all. Patents especially are difficult to navigate because the exclusivity that is afforded by them is discretionary. That is, a patent holder can choose to exercises their rights when and where they like, leading to what has been termed Patent trolling or Submarine Patents where a patent holder does not enforce their exclusive rights until another organisation or individual becomes profitable or exposed (via marketing or public recognition). Whole business

\footnotetext{
12 These summary documents are available from the ministries website http://www .med.govt.nz
} 
methods around this practise are increasingly common. How a company responds to these challenges, to determine the legitimacy of a claim, and whom they employ to mitigate this risk, and further who is authoritative in determining a decision are important themes, not only to industry but to the supporting legal structures and profession around them.

As this theme appears generally as an adjunct to other matters, it is important to identify which other themes and fields of interpretation are used to move into the general theme of authority and legitimacy. In most submissions there is some discussion of the role patent attorneys have. But one of the more discussed features of the New Zealand patent act is the process and power to overrule or impose conditions on a particular patent. Generally discussion is grounded, for the most part, in the traditional legal hierarchical system and courts. However there are some notable suggestions for both the application process, and screening of patents which challenge the traditional hierarchy of patent attorney, patent examiner, patent commissioner and courts. This challenge comes from how third party objections are allowed, and even more radically in the examination procedure, where there has been some suggestion and trials in other countries of a distributed patent examination process, harnessing something akin to collective intelligence using public participation in patent examination by recruiting distributed technical expertise into trademark and other IP examination procedures. Currently there are trials of this type of system by the USPTO, and their have been several private companies doing similar prior art searches using similar methods to enhance quality of legal advise and patent application processes ${ }^{13}$. It is important to also note that their have been a number of high profile legal cases such as the long running SCO vs NOVELL lawsuit in the US where community participation and research into patents and prior art have greatly assisted discovery ${ }^{14}$ (although this situation is slightly different). Within New Zealand, nonprofit organisations such as the New Zealand Open Source Society ${ }^{15}$ have

\footnotetext{
${ }^{13}$ See Public participation in patent examination - Wikipedia, the free encyclopedia, n.d. for a review of public participation in patent processes, and Schecter and Murray (2007)

${ }^{14}$ (Groklaw - Barracuda Networks Asks For Help Finding Prior Art to Defend ClamAV - Updated $3 X s$, n.d.)

${ }^{15}$ Website http://www.nzoss.org.nz
} 
conducted similar distributed prior art discovery research processes, and have been successful in having at least one notable patent application dismissed before examination based on their research ${ }^{16}$.

The following related tracts come from a legal grouped submission, emphasising biodiversity and plant variety issues in response to question of authority of oversight in patent process

The grant of a patent has its basis in social contract... essential that commissioner of Patents retains his/her power to refuse a patent on the grounds that it is contrary to morality... decisions are open to challenge in the High Court.

This is in relation to the issue of whether there is a high level veto power invested in an patent system oversight individual. The submitter argues that this should be the case, and implicates the morality repertoire. Further that this veto power is not final. The submitter goes on to further support allowing for arbitration powers

By removing the discretion, patent applicants and objectors are left to bring an application to courts... Settling matters by precedent is expensive and with the onus then being on individuals to protect the issues, there would be a significant reduction in public protection.

The submitter is defining protection in this instance, not of rights holders/applicants, but in terms of public good, by implying that there is inadequate understanding and resources to identify abuses and exploitation of the patent system in the general public. This is reinforced again in terms of what is required to apply for a patent, at the same time standardising the role and authority that the legal profession must have in a patent application process.

Patents are inherently legalistic and require the applicant to employ a patent attorney to draft claims.

Overall the submission constructs patents as being inherently bad for the public good, in relation to high-technology fields and in reference to

\footnotetext{
${ }^{16}$ (Computerworld > Government ponders digital media regulation, n.d.; Computerworld > UK looks to relax restrictive copyright laws, n.d.)
} 
bio-technology, plant varieties and genetic resources. The submitter emphasises a number of international treaties in relation to plant varieties that are outside of the current established frameworks such as TRIPS and GATT, and proposes that the regulations imposed by these treaties, which are concerned primarily with growers and breeders rights, offer more appropriate protections. There are repeated warnings in the submission in relation to bio-diversity, economic and market diversity if plant/gene and biotechnology exemptions are not implemented. Overall the demonstration of authority is that there needs to be checks, and that patents are inherently legal in nature, effectively excluding the notion that patents are accessible to all, by explicitly referring to patent claimants as being an elite, and generally outside of the the public's comprehension. The themes of this particular submitter seem to argue towards an industry specific authority in decision making as much as it marginalises and de-emphasises any greater public incorporation into the process of review of the controls.

A contrasting legal submission which is more representative of the legal submissions as a whole, comes from a submitter who makes extensive claims to represent patent attorneys both in New Zealand and Australia. Unlike the previous submission it does not acknowledge explicitly any risk or dangers to the public posed by increased patent protections, but uses an investment/economic incentive, stake claim. The submitter explicitly makes claims against the legitimacy of including exemptions of certain fields, such as biotechnology, plant varieties and medical methods. The main way in which the submission supports these arguments is through the use of the harmonisation theme and related discourses. In addition to this they place extended influence on harmonisation between Australia and New Zealand, and make many claims that New Zealand should effectively adopt the provisions in Australian law.

Thus, on the theme of authority and legitimacy, there is a definite push from legal-economic submissions towards keeping the authority of maintaining standards to relevant specialised industry participants. This is significant in that it associates legitimacy exclusively with an Industrial conception of knowledge and ICW, and in some submissions, raises concerns of any oversight power from public interests (such as the concerns raised 
by legal firms about morality clauses included in the act, and the right to veto patent applications being invested with the Patent Commissioner). The emphasis on removing oversight from public conceptions of knowledge is important as it lends further weight to a clustering of specific trade based conception of knowledge that supports a conception of knowledge that is anchored in traditional physical good concepts.

\subsubsection{Inventor, Novelty and Innovation}

This theme is included because it was included explicitly in the official ministry report on public submissions to the patent act draft. As a representational field and generalised theme it concerns the points raised in submissions positions on how inventor is defined. Importantly the topic of inventor is almost inextricably linked to topics of novelty and innovation as much, if not more so than what constitutes an inventor. These topics are rarely visited alone in submissions but clustered together. In terms of reified legal wording, currently in New Zealand law there is one overriding phrase which is linked to claims of novelty and innovation, this is repeated in legal submissions most often, but occasionally appears in submissions from other groups. This statement/phrase is method of new manufacture. This single statement is used as the benchmark for not just novelty but also as the baseline argument for subject matter that is considered patentable. In effect it is acting as the guiding recognised legitimising referent anchor for subsequent discussion. Thus it has influence as an effective institutional transmitted symbol on further discourses and on construction of a social object around inventors, novelty and innovation.

From the official draft bill submission summary document as issued from the Regulatory and Competition branch of the ministry of economic development.

The draft Bill carries over the term "true and first inventor" from the Patents Act 1953. A number of submissions have pointed out that judicial interpretation of this term includes a person who first introduced or imported an invention into New Zealand even though the person had no part in actually devising the invention. This interpretation is facilitated by the "local novelty" provisions of the current 
Act. "Invention by importation" is a concept that no longer features in the patent law of other developed countries, and it was never the intention to retain the concept in the Patents Bill. It has largely been rendered obsolete by the move to "absolute novelty" standards internationally. Under an absolute novelty standard, public disclosure of an invention anywhere in the world by any means before a patent application is filed destroys its novelty and would prevent the grant of a patent. The Patents Bill incorporates an "absolute novelty" standard. Another factor is that the term "true and first inventor" may give the misleading impression that New Zealand grants patent rights on a "first to invent" basis. This is not the case, patents in New Zealand are granted on a "first to file" basis. In light of these factors, and to remove any doubt, the Ministry proposes that the term "true and first inventor" be replaced by the term "inventor". In addition, the Ministry is also considering including a definition of "inventor" into the Bill. Such a definition would look to define the inventor as the actual divisor of the invention. The term "local novelty" in this context means that an invention is considered new if a description of the invention has not been published in New Zealand, or the invention has not been used in New Zealand before the date the patent application was made. Use or publication of the invention outside New Zealand before this date would not prevent the grant of a patent in New Zealand.

The main observation in terms of social objects of inventor and invention is that the official summary position makes specific reference to abstracting the concept further away than current wording in the existing bill concedes, thus we see the conceptual "Cowboy Inventor" myth as encountered in many lay constructions, of how patents provide benefits for societies and individuals, being marginalised. This is a logical progression moving away from perceiving individuals as being a significant component in the regulatory rights system, and placing further emphasis on groups and organisations as being primary utilisers of the patent system. It is also important to note that the rights system automatically must be structured to exclude publicly or community developed innovations, while this is not revelatory, in that the patent system has never in- 
corporated community or public development, the fact that no effort to include ways of dealing with these novel methods of communal development/innovation which has become a significant approach, in wake of the enabling ICT infrastructure, is telling of the expected sort of conceptual development models the patent system is setup to support, and further those which it marginalises and discriminates.

\subsubsection{Drafting Issues/Legal wording}

This theme requires a note because it forms an important chunk of the submissions, and is afforded more attention than any other theme in the summary document of the 2 nd round of submissions, with the volume of pages dedicated to it in the submissions, largely coming from legal firms. While somewhat uninteresting from a social representation point of view, it belies a bureaucratic theme which is important to understand because it permeates regulatory decisions. In-line with Moscovici's (Moscovici \& Markova, 1998) conception of reified worlds, with their own internal jargon that works to the exclusion of outside groups, the legal-political wording present in the legal and industry submissions primary, can be seen to serve the same purpose. In both summary documents those submissions which were observed to draw attention to specific legal technical wording and jargon were given the most coverage, and bore closest to the positions recommended by the government reviewers.

\subsection{On the role of conflict and contradictions}

Many of the submissions are in direct opposition to each other in terms of both the treatment of themes and the use of language. For instance most of the Legal submissions continue to use highly reified legal language throughout the submissions, and make very specific syntactical suggestions and alterations, which are at an advantage in terms of being integrated into the officially mandated overviews and summary documents. That puts other submissions, which make broader, yet sometimes as detailed references to international treaties and issues of public and national 
advancement, in relation to the patent system and patent act in general, at a disadvantage. In terms of conflict, the way in which this opposition is negotiated is that the latter is essentially ignored when it does not fit the agenda defined by the bureaucratic procedure. This is most clearly evident in that the actual draft bill following the analysis of the first round submission, which fails to include many of the points raised by non-legal entities, while they are given cursory acknowledgement in submission overview documents. As for the second round any challenge to the established economic-legal representation as constructed primarily through the reliance of international obligations with explicit highlighting of the TRIPS agreement is excluded from government commission analysis proceedings, despite the second round following the publication of the draft bill attracting far more detailed submissions. In terms of how conflict is used to justify this, first stake is constructed as being principally, the hypothetical investment of funds by the establishment of research and development industries in New Zealand, especially the pharmaceutical and bio-technology industries. Conflict is widely operationalised both in submissions and in official commission reports as the conflict arising from New Zealand deviating from overseas practises and International agreements, and in turn hypothetical risk to potential investment ( and sometimes the threat of trade sanctions). The main countries which are used extensively for comparison are The UK, Australia and the USA and occasionally the European Union is mentioned. However, because the EU has adopted a number of deviations from both the UK and USA's patent systems, primarily in terms of exclusions, and the varied positions member states have towards IP laws it is less frequently referred to by proponents of this operationalisation. Contempt is actively shown for alternative conceptions which argue for deviations or exemptions in a number of submissions and reports, even where there is ample evidence provided to support those alternative conceptions or for those exclusions.

It is clear the there is a dominant representation which is used to influence the political system, one which is resilient to change and which exemplifies a classical neo-liberal economic position towards the increased commodification of almost all types of intellectual and creative work that 
falls towards the invention/idea/method spectrum. This is structurally resistant to change regardless of the consultation processes which are inherent in a democratic society such as New Zealand. This position regardless is in direction opposition of publicly presented rhetoric appealing to innovative/public good/knowledge economy discourses, or as encountered in the likes of the following Patent Act Draft review, media release.

A modern and effective patent law provides an incentive for innovation, while maintaining an appropriate balance between the interests of innovators and the interests of users of patented technology. - Judith Tizard

As shown throughout this chapter, themes and positions are demonstrably constructed in a fairly singular fashion which is not guided by principles of rewarding innovative individuals or groups, at least not those that exist outside of what could be considered an Industrial social representation of ICW. In effect those recognised and incorporated into a legal reified social representations of knowledge are those that aim specifically at producing a legislative construct that minimises the distinctiveness of New Zealand in a globalised economic business and trading environment, providing a streamlined legal operating framework for associated legal industry and to maintain and strengthen current traditionally grounded industrial representations of knowledge production and consumption. The relative exclusion of a collective participatory representation from these themes unfairly positions these groups into a consumer frame and, as already hinted at, this is far from an accurate depiction of the groups outside of large industry, nor is it representative of all industry. It is no wonder that these expanding groups have become so vocal, or that these currently in power groups are seeking to expand and maintain protections at what must seem like a threat.

\subsection{So where is the Property? Summary}

Within the historical and literature review discussed in previous chapters, patents are the form of right which has been inferred to be most closely 
linked with the property metaphor. But within the data set presented here it is seldom explicitly invoked as a theme. Why might this be? Or is the property conception simply hiding?

If we apply the proposed historical development of social representations associated with control of ICW as presented in chapter 3, there is a definite period where Patents were considered the most linked to the physical property anchor. However I contend that it has become so objectified within a trade and industrial social object that it has become almost a cliché to invoke this within groups that follow a trade related role. Groups which exist outside of this sector of activity have for the second half of last century occupied a consumer/user positioned by the Industrial legaleconomic dominance of multinationals and in turn economic development boards and related industry groups nation-states focus on a neo-liberal free market positivism for the development of society. However digital media and the technologies of the network have progressively liberated these groups from this position by breaking away from a simple passive interaction with the conceptual phase space as distributed via Industrial representational subscribers. Enabled by the ease at which groups could be formed, and information retrieved, a surge in tangible outputs from the "Public" has become a significant and recognisable component of people's everyday lives. For this group the concept of knowledge has become diversified, but I think the primary feature is that most people have come to position themselves in a participatory role. Property is still important, but it has once again become individual in nature, and with it a recognition that an individual's knowledge comes from many sources, thus an individual ICW is collective in its origin. Industry, rather than acknowledge this representation directly, shows signs of moving towards a representation of knowledge as not just being produce, but as being strategic.

Figure 4.2 shows an updated thematic map extending on the historical representations developed in chapter 3. It follows the same principle of incorporating a time-line element. I have also superimposed what I consider to be the most important technological events which have influenced upon the development of the representations. 


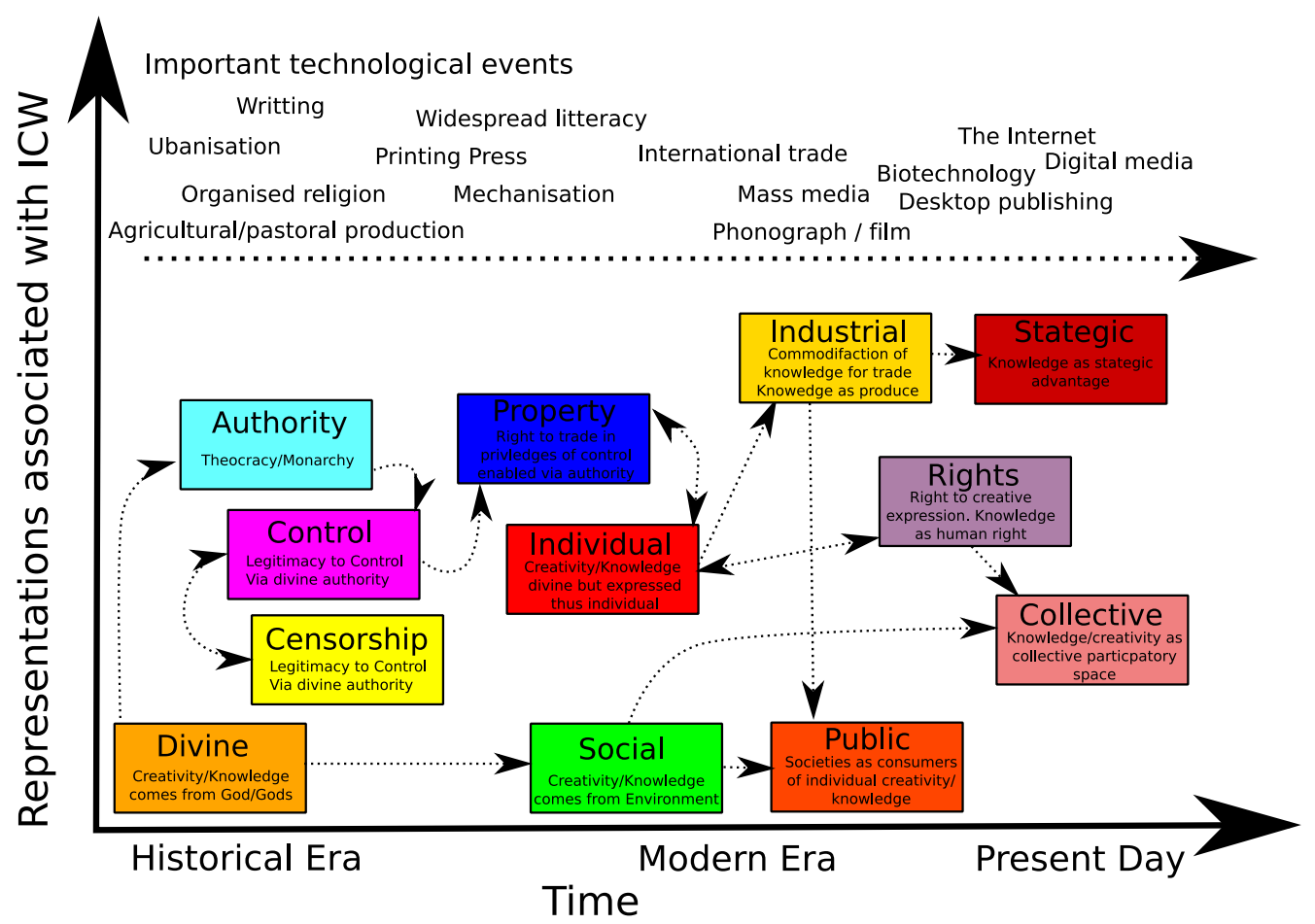

Figure 4.2: Proposed expanded map of social representations related to Intellectual and Creative work through time, including important technological developments, and various influences between representational objects development

\subsection{Informing Social Representations Theory}

The approach taken here applying thematic analysis, and in particular thematic maps, as tools for investigating social representation is probably the most significant addition to SRT, and has the benefit of allowing for social groups and those groups social representations to be positioned in relation to others. The use of a narrative historical framework to position these current social objects is also a key component to helping gain insight into how and where various subject mater positions have come from, and for making projection as to how they may change in the future. This time line element to social representation is discussed by previous authors such as Bauer and Gaskell (1999), however to this authors knowledge the incorporation of such a wide ranging time period for examining social objects is a 
first, and can only be made possible using the sort of narrative combinational mixed study approach used here.

Referring to 4.2 and presuming that the historical representations suggested in the previous study can be taken as indicative of common sense understanding (or at the very least, informed positions) through history, we can construct an evolutionary approach to social representations of ICW.

The current data set suggests that there is a re-assertion of what is could be regarded historically as natural rights or in other cases socially produced/common knowledge. It would follow that at some point in recent history that these rights had been eroded. If we look at the progression we can observe that there has been two main streams to which social representations have been aligned to traditionally. With the individual rights discourses being clearly supplanted in recent history by industrial conceptions as the authoritative/legitimate representation, it is through this stream that the largely undefended majority position (if we consider the majority to be those without an opinion)have been labelled/positioned to have a common sense representation of ICW as being a product, thus as a public of consumers directly in relation to the industrial representation. However with recent technological and social developments this undefended common sense position has come to be re-discovered as the masses find themselves being drawn into contact with legal conceptions supporting the dominant industrial conception and disagreeing with it.

In this way we can observe how collective symbolic coping occurs, within a historical context which enables a better understanding of how anchoring and objectification may occur, simply because historical representations and symbols are most likely to be mobilised as groups which carry components of these historical narratives and representations mobilise them in public discourses.

I suggest more emphasis should be given, to longer ranging historical aspects surrounding any given topic of investigation employing social representations theory in general than is currently afforded by researchers. 


\section{Chapter 5}

\section{Review and Concluding remarks}

Personal Tabu: A small rule for living, bordering on a superstition, that allows one to cope with everyday life in the absence of cultural or religious dictums. - Douglas Coupland, "Generation X: Tales for an Accelerated Culture"

\subsection{Concluding remarks}

As I have been investigating and writing this thesis I have been presented with the difficultly of trying to bridge four distinct academic areas, Psychology, Computer Science, Economics and Law. Apart from having to learn many basic and advanced economic and legal theories early on to grapple with the literature, one of the key features of this process discovered very early on is that researchers among all of the disciplines of law, economics computer science, on the topic of IP all approach similar fundamental issues from different disciplinary background, but sharing a common defining feature. And that was the centrality of the cultural phenomenon of the network ${ }^{1}$, not just as a tool, but as occupying a greater importance in all their respective research and life in general. As the reader would have no doubt come to understand, I too have come to occupy a similar position, in that I position the network as being the defining social change spurring many of the issues discussed here and refuelling old debates gone cold. One part of a discipline that has failed to grapple with emerging themes centred around the network has been Cross-cultural and Social psychology. While there has been efforts in the wider discipline, in

\footnotetext{
${ }^{1}$ as it relates to technologies of distribution, communication and digital media enabling group and community formation
} 
the form of journals like the Journal of Computer Mediated Communication $^{2}$, that have gone from obscurity to prominence in the last 5 years. The JCMC is an exception, however, having been originally grounded in linguistic communications and discursive psychology methodology, its more recent contents has come to resemble more the sort of Indigenous Psychology of the network which has traditionally appeared and been catered to by non-discipline specific journals like First Monday ${ }^{3}$ that have focused on society and technology issues, and have always had a primary focus on the network. All of this activity and the highly salient influence the network has on our day to day lives leaves me somewhat amused to attend seminars or read comprehensive cross cultural literature examining 30/40/50 cultural groups in classic cross cultural comparative style, while ignoring the influence the network has had on redefining identity, group associations and development, as more and more individuals find themselves detached from political geographical indicators, and traditional conceptions of spatial segregation lose meaning. Understanding the constituent parts of this Digital Cultural Identity should be an important part of the psychology discipline, and should be mainstreamed into research paradigms. Just as Yugoslavia was dissolved, reformed, re-established and dissolved into separate nation states, each represented a change in how to incorporate the new labels in any study looking at Eastern Europe, so should the network be treated to the same conceptual inclusion and redefinition process within psychology.

How is this related to social representations and Intellectual and Creative Work? Well, as observed both in the literature review and the patent act study, the network is central in enabling navigation of inherently abstract segments and chunks of information. Without the agnostic, plentiful, vast, instantaneous carrier medium of the network it is harder to communicate the conceptual representation of knowledge and ideas between groups and individuals. The network which, although technically a bunch of wires, fibre, Integrated circuits, control protocols and such,

\footnotetext{
${ }^{2}$ Which recently was involved in a fairly instrumental deal with a major academic publisher allowing it to continue its liberal publishing/open access format while being incorporated into the publishers stable

${ }^{3}$ http: //firstmonday.org
} 
has provided a salient and accessible anchor for the objectification of the process of sharing, accessing and retrieving and most importantly participating in the act of abstract knowledge and information conveyance and procurement. Without the network as a model for common sense understanding, as the reference point, any group which might have constructed abstract knowledge in this fashion would have been hard-pressed to explain it to outside groups. This might explain why mathematicians, computer scientists engineers, hackers, academics, artists and other abstractthinking groups traditionally associated with the "social-nature" social representations of knowledge/ICW, have been the biggest supporters of a societally endorsed re-representation of Intellectual property rights. In the wake of the network having become so embedded and central to everyday life throughout society (a brute fact as it were), there has been a new widespread awareness of participatory positions that each of us as human beings can have in accessing and adding to the conceptual space of thought, by simply accessing the network. A hypothetical example best illustrates:

Ten years ago a middle aged farmer had few avenues to engage a collaborative store of knowledge, let alone participate and add to it (without significant barriers to entry). This individual may not have taken a book out of a public library since primary school, seldom engaging the phase space of thought more than engaging friends and relatives in conversation, and dealing with issues on the farm, or flicking the channel on the TV. Now the same hypothetical individual, while not an avid user of the network, at least participates, checks e-mail and information on-line, and is a member of a mailing list of farmers who swap information on stock feeds and other relevant information.

This vignette can be swapped for hundreds if not thousands of similar cases of slightly differing content and context, even in cases where the individual may not use the network themselves, they are likely to have an understanding (even if limited), as gleaned through interactions with individuals or activities where the network is Incorporated.

The objectified conceptual idea of the network as a vast, instantaneous 
agnostic $^{4}$ carrier of information, as much as the physical medium digital communications and storage technologies, produces the key element in why status quo legal representations have come to perceive themselves as needing more legal protections and rights so as to maintain histori$\mathrm{cal} /$ current positions of status and power.

On one side of this argument are groups which benefit from the current representations of the conceptual space - i.e. remaining anchored in an individual and property metaphor, providing support of an Industrial object. There are also groups that also can be included here, that have slipped by the wayside in this analysis - the groups that have been far removed from the common sense representation and theme of the network, and so have been able to maintain there own exclusive social objects of the network. There are still societies and groups around the world which, for the time being at least, continue to exist in participatory vacuums, including traditional groups, third world citizens, and the underprivileged. For these groups, the conception of knowledge in relation to the globalised world remains one of a consumer and user of industrial groups produce. While it is possible many of these groups may hold social representations of ICW as divine or social, the legitimacy of these conceptions, as demonstrated throughout this thesis, is largely ignored by the status quo. Projects like 'One Laptop per Child' may help make the shift for these groups, but for the Industrial groups themselves only time will tell. Already, some industries have moved to embrace the collectivist conception of knowledge, finding the economic methods of extracting value from these representations a goldmine. Getting the dominant groups to adjust there business models en-masse however is not likely to occur any time soon, again only time will tell.

As for the rest of us, the future is somewhat uncertain. I argue that the approach taken to writing exceptions into existing legal paradigms to account for the new liberalised collectivist subscribing groups in society is

\footnotetext{
${ }^{4}$ For the most part. While attempts in some geographical/political regions, are marginally successful at censoring elements of the network (notably the world wide web (hypertext)), by using both language and technology to demarcate barriers/discourage activities, there are always ways around such censorship due to the underlying nature of the technology
} 
the completely wrong way to approach the issue. More suited would be the establishment of a set of legislative frameworks around fair use, like the much-touted United States Freedom of Speech constitutional clause. This would be more suited to how most groups in society go about using the conceptual phase space in day-to-day life. In any event, I see two worlds emerging, one dominated by legal restrictions and lawsuits, licensing agreements and defensive portfolios of rights for doing business, and another which mostly ignores the legal rights, as something "for the big guys". Where to draw the line in what constitutes a commercial bit of knowledge which should be subject to the rights world is the hard part. Currently Free Software is hitting that exact legal situation very hard, having been so successful at circumventing the rights structures, and doing so in spite, not because of them. It seems utterly contradictory to the general theme of what is meant to be at the heart of these rights, to slap down obviously innovative and commercially stimulating endeavours that exist outside rights frameworks, just because some company, individual or co-operative managed to secure legal rights to something, whatever it may be. While software is the consistently used example because of its salience, many of the arguments are equally applicable to other endeavours given the technologies of the network are spreading to all sectors of human endeavour, software was just there first due to mathematics integral role within digital electronics and signalling.

In final conclusion, I shall restate the original goals and answer them in reflection.

Goal: To identify if there are social representations of a globalised concept of abstract knowledge, if so how do different groups realise it?

Yes, I argue that there are definite social representations of abstract knowledge, the two groups that have emerged are industrial and greater society ${ }^{5}$. Industry clearly mobilises a social representation centred around trade and commercial interests and as such it uses a conceptual anchor of physical property for much of its interactions with the world around it. For the rest of us, until recently there has been a position among the dominant Industrial groups representation, a product of that group both hav-

\footnotetext{
${ }^{5}$ acknowledging that this conception puts industry in a minority position
} 
ing influence on authority figures, and controlling distribution channels. However the technologies of the network have changed this representation for groups outside of large industry, to incorporate a participatory element that has changed distributional power structures, leading to a rerepresentation of abstract knowledge into a collectivist conception.

Goal: To identify elements of the social representations and associated themes present in modern day social objects as well as attempt to identify historical representations of ICW.

Historically there has been what appears to be a Divine initial representation, which incorporated religious components. This developed over time and the controlling structures associated with hegemonic structures in developing societies led to a privilege system, which in turn led to a social and environmental origin conception of knowledge, at the same time as developing legitimacy for individual rights associated with privileges with the advent of industrialism. This in turn led to a property discourse revolving around control themes. The social/environmental representation however continued to be the guiding legitimising structure around the control themes and splits away into an industrial conception. Eventually the social nature component fades and is replaced with a public as a consumer of knowledge products, during a period of heavy commodification of many types of industry (industrial revolution, and then mass media).

Goal: Identify groups which claim to have stake and if present, the features of conflicting and contradictory representations, and how and why contradictory representations are held. The main group which has invested stake are groups which subscribe to an Industrial/knowledge production representation of knowledge. These groups, industry, media, legal and by nature of the influence these groups exert political, are in conflict with almost all representations of knowledge that exist outside of this commodified approach to knowledge. They value the scarcity that IP rights systems introduce as it enables them to profit from trade in them. Any representations of knowledge outside of this tight representation could be perceived as a threat because they potentially de-commodity segments of conceptual phase space that these groups have become de- 
pendant on to operate.

\subsection{Final Thought}

While the themes presented here seem to construct the current technological developments as an epiphany in human development, and suggest a huge social and cultural upheaval due to changes in societies, there is a thought in the back of many peoples minds, that despite the obvious changes in all our day to day lives, that the flirtation with collectivist non free-market conforming treatments of IP is a passing fad. There is rhetoric of communism (which has only negative connotations in most people's minds), thievery and unsustainability in current practises. I would suggest to people who fence sit, rather than take sides, or to businesses attempting to grapple with these issues, that now is the time to start to ignore the laws as they relate to IP rights, not by breaking them, but rather avoiding them by crafting business models which do not draw upon the dominant conceptions, as this is clearly going to give you advantages over those that buy in and have the overhead of dealing with the rights systems, now that there is a number of supporting structures and technologies to do so it is no longer an unproven path, nor are you alone. Many movements, like Creative Commons, GNU foundation and others have created exquisite legal loopholes to flaunt the purpose of the laws which they have found archaic and ill suited to incorporating development and creative endeavours that arise from the technologies of the network. So go and use them. 


\section{Bibliography}

Alford, W. (1997). To steal a book is an elegant offense: Intellectual property law in chinese civilization. Stanford University Press.

Allred, B., \& Park, W. (2007, November). Patent rights and innovative activity: evidence from national and firm-level data. Journal of International Business Studies, 38, 878. Available from http://proquest.umi.com/pqdweb?did=1369553391 \ $\& \mathrm{Fmt}=7 \backslash \& \mathrm{Cl}$ ient $I d=7511 \backslash \& \mathrm{R} Q \mathrm{~T}=309 \backslash \& \mathrm{VName}=\mathrm{P} Q \mathrm{D}$

Antaki, C., Billig, M., Edwards, D., \& Potter, J. (2003). Discourse analysis means doing analysis. Discourse Analysis Online, 1.

Aronson, J. (1994). A pragmatic view of thematic analysis. The Qualitative Report, 2,. Available from http://www. nova.edu/ssss/QR/ BackIssues/QR2-1/aronson.html

Atkinson, J. M., Heritage, J., \& Maxwell, J. (1984). Structures of social action: Studies in conversation analysis. Cambridge UniversityPress.

Augoustinos, M., LeCouteur, A., \& Soyland, A. J. (2002). Self-sufficient arguments in political rhetoric: constructing reconciliation and apologizing to the stolen generations. Discourse \& Society, 13, 105.

Augoustinos, M., Walker, I., \& Donaghue, N. (2006). Social cognition: An integrated introduction (Second Edition ed.). Sage Publications Ltd.

Baerveldt, C., \& Voestermans, P. (2005). Culture, emotion and the normative structure of reality. Theory \& Psychology, 15, 449.

Bangerter, A. (1995). Rethinking the relation between science and common sense: a comment on the current state of social representation theory. Papers on Social Representations, 4.

Barr, P. (2003). User-interface metaphors in theory and practice: A thesis submitted to the victoria university of wellington in fulfilment of the requirements 
for the degree of master of science in computer science.

Bauer, M., \& Gaskell, L. (1999, June). Towards a paradigm for research on social representations. Journal for the Theory of Social Behaviour, 29, 163-186. Available from http: / / www. ingentaconnect. com/ content/bpl/jtsb/1999/00000029/00000002/art00096

Bekar, C., \& Lipsey, R. (2001). Science, institutions, and the industrial revolution. Oxford University Press. Available from http://www .sfu.ca/ $\{\sim$ \}lipsey/davis.PDF

Benkler, Y. (2000, May). From consumers to users: Shifting the deeper structures of regulation toward sustainable commons and user access. Federal Communications Law Journal, 52, 561.

Benkler, Y. (2002, December). Coase's penguin, or, linux and the nature of the firm. The Yale Law Journal, 112, 369.

Benkler, Y. (2004, August). Commons-based strategies and the problems of patents. Science, 305, 1110. Available from http: / / proquest . umi. com/pqdweb?did=684900361 \\&Fmt= $7 \backslash \& \mathrm{Cl}$ ient $\mathrm{Id}=7511 \backslash \& \mathrm{RQT}=309 \backslash \& \mathrm{VName}=\mathrm{P} Q \mathrm{D}$

Billig, M. (1991). Ideology and opinions: Studies in rhetorical psychology. Loughborough studies in communication and discourse. Thousand Oaks, CA, US: Sage Publications.

Billig, M. (1996). Arguing and thinking: a rhetorical approach to social psychology. Cambridge University Press.

Boldrin, M., \& Levine, D. K. (2008). Against intellectual monopoly. Cambridge University Press. Available from http://www.dklevine .com/general/intellectual/againstnew.htm

Boyatzis, R. E. (1998). Transforming qualitative information: Thematic analysis and code development. Sage Publications Inc.

Braun, V., \& Clarke, V. (2006, April). Using thematic analysis in psychology. Qualitative Research in Psychology, 3, 77-101. Available from http://www.ingentaconnect.com/content/ sage/qrp/2006/00000003/00000002/art00002

Byford, J. (2002). Anchoring and objectifying "neocortical warfare": Representation of a biological metaphor in serbian conspiracy literature. Papers on Social Representations Textes sur les représentations so- 
ciales, 11. Available from http://www.psr.jku.at/PSR2002/ 11_3Byfor.pdf

Carlaw, K., Oxley, L., \& Nuth, M. (2006). Beyond the hype: Intellectual property and the knowledge society/knowledge economy. Journal of Economic Surveys, 20.

Chartrand, H. H. (2000). Copyright c.p.u.: Creators, proprietors, and users. Journal of Arts Management, Law, and Society, 30, 209. Available from http://proquest.umi.com/pqdweb?did=67546490 \ \&Fmt $=7 \backslash \& \mathrm{client} I \mathrm{~d}=7511 \backslash \& \mathrm{RQT}=309 \backslash \& \mathrm{VName}=\mathrm{PQD}$

Christensen, C. M. (1997). The innovator's dilemma: when new technologies cause great firms to fail. Harvard Business School Press.

Clémence, A., Devos, T., \& Doise, W. (2001). Social representations of human rights violations: Further evidence. Swiss Journal of Psychology Zeitschrift für Psychologie - Revue Suisse de Psychologie. Vol 60(2).

Clémence, A., Doise, W., Rosa, A. S. de, \& Gonzalez, L. (1995). La représentation sociale des droits de l'homme: Une recherche internationale sur l'étendue et les limites de l'universalité. International Journal of Psychology, 30, 181. Available from http://www.informaworld .com/10.1080/00207599508246565

Cohrs, J. C., Maes, J., Moschner, B., \& Kielmann, S. (2007). Determinants of human rights attitudes and behavior: A comparison and integration of psychological perspectives. Political Psychology, 28, 441469. Available from http://www.blackwell-synergy.com/ doi/abs/10.1111/j.1467-9221.2007.00581.x

Computerworld $>$ government ponders digital media regulation. (n.d.). Available from http://computerworld.co.nz/news.nsf/news/ AF2BEF0AAFB8C732CC2573DA000232C1?opendocument \\&utm _source=topnews $\backslash \& u t m \_m e d i u m=e m a i l \backslash \& u t m \_c a m p a i g n=$ topnews

Computerworld $>$ uk looks to relax restrictive copyright laws. (n.d.). Available from http://computerworld.co.nz/news.nsf/news/ DB71D10BC88D7E21CC2573CA0066E246?opendocument \\&utm _source=topnews $\backslash \& u t m \_m e d i u m=e m a i l \backslash \& u t m \_c a m p a i g n=$ topnews 
Cox, P. A. (2000). Essay on science and society:will tribal knowledge survive the millennium? Science, 287, 44-45. Available from http:// www. sciencemag.org

Diamond, J. M. (1999). Guns, germs, and steel: The fates of human societies. Norton.

Diaz-Veizades, J., Widaman, K. F., Little, T. D., \& Gibbs, K. W. (1995). The measurement and structure of human rights attitudes. Journal of Social Psychology. Vol 135(3), 135, 313-328.

Doise, W. (1993). Debating social representations. New York, NY, US: Clarendon Press/Oxford University Press. (.

Doise, W. (2001). Human rights studied as normative social representations. Deaux, Kay; Philogène, Gina (2001). Representations of the social: Bridging theoretical traditions. (pp.

Doise, W., Spini, D., Jesuino, J. C., \& Ng, S. H. (1994). Values and perceived conflicts in the social representations of human rights: Feasibility of a cross-national study. Swiss Journal of Psychology - Zeitschrift für Psychologie - Revue Suisse de Psychologie. Special Issue: Structuring conflicts. Vol 53(4), 53, 240-251.

Drahos, P., \& Smith, H. (1999). The universality of intellectual property rights: Origins and development. In Panel discussion on intellectual property and human rights. Geneva: WIPO. Available from http://www.wipo.int/tk/en/hr/ paneldiscussion/papers/pdf/drahos.pdf

Durkheim, E. (1973). On morality and society (R. N. Bellah, Ed.). University of ChicagoPress.

Elejabarrieta, F. (1994). Social positioning: a way to link social identity and social representations. Social Science Information, 33, 241-253. Available from http://ssi. sagepub.com

Eliade, M. (1961). Images and symbols: Studies in religious symbolism. Princeton UniversityPress.

Farr, R. (1987). Social representations: A french tradition of research. Journal for the Theory of Social Behaviour, 17, 343-365. Available from http://www.blackwell-synergy.com/doi/abs/ 10.1111/j.1468-5914.1987.tb00103.x 
Farr, R. M. (1993). The theory of social representations: whence and whither? Papers on Social Representations, 2, 130138. Available from http://www.psr.jku.at/PSR1993/ abstracts1993/2_17abstr.html

Finkel, N. J., \& Moghaddam, F. M. (Eds.). (2005). The psychology of rights and duties: Empirical contributions and normative commentaires. The law and public policy. Washington, DC, US: American Psychological Association. (.

Fluid - wikipedia, the free encyclopedia. (n.d.). Available from http://en -wikipedia.org/wiki/Fluid

Gannon, M. J. (2001). Cultural metaphors: Readings, research translations, and commentary. SAGE.

Gaskell, G., \& Bauer, M. W. (2001). Biotechnology 1996-2000: The years of controversy. NMSI Trading Ltd.

Golub, A. (2004). Copyright and taboo. Anthropological Quarterly, 77, 521-530. Available from http://muse.jhu.edu/journals/ anthropological_quarterly/v077/77.3golub.html (Volume 77, Number 3, Summer 2004)

Gowers, A. (2006). Gowers review: of intellectual property. Available from http://www.hm-treasury.gov.uk/independent_reviews/ gowers_review_intellectual_property/gowersreview _index.cfm (Gowers Review: index)

Groklaw - barracuda networks asks for help finding prior art to defend clamav - updated 3xs. (n.d.). Available from http://www.groklaw. net/ article.php

Guanhong, T. (2004). A comparative study of copyright and the public interest in the united kingdom and china. SCRIPT-ed, 1:2, 272. Available from http://www.law.ed.ac.uk/ahrc/script -ed/issue2/china.asp

Haile, H. G. (1983). Luther: An experiment in biography (New ed.). Princeton Univ Pr.

Hart, O., \& Moore, J. (1990, December). Property rights and the nature of the firm. The Journal of Political Economy, 98, 1119-1158. Available from http://links.jstor.org/sici?sici=0022-3808\% 
$28199012 \div 2998 \% 3 A 6 \div 3 C 1119 \% 3 A P R A T N O \div 3 E 2.0 . C O \div 3 B 2-3$

Heller, M. A., \& Eisenberg, R. S. (1998, May). Can patents deter innovation? the anticommons in biomedical research. Science, 280, 698-701. Available from http://www.sciencemag.org/cgi/content/ abstract/280/5364/698

Herder, M., \& DyckBrian, J. (2008). Canada's stem cell corporation: Aggregate concerns and the question of public trust. Journal of Business Ethics, 77, 73. Available from http://proquest . umi . com/pqdweb?did=1387346731 / $\& \mathrm{Fmt}=7 \backslash \& \mathrm{Cl}$ ient $\mathrm{Id}=7511 \backslash \& \mathrm{RQT}=309 \backslash \& \mathrm{VName}=\mathrm{PQD}$

Herrera, M., Lavallée, M., \& Doise, W. (1998). Représentations sociales des droits de l'homme auprès de diverses communautés culturelles au québec. / social representations of human rights among culturally diverse communities in quebec. Revue Québécoise de Psychologie. Vol 19(3), 19, 151-187.

Hesse, C. (2002). The rise of intellectual property, 700 b.c.-a.d. 2000: An idea in the balance. Daedalus, 131, 26. Available from http: //proquest . umi. com/pqdweb?did=121881925 \\&Fmt= $7 \backslash \& \mathrm{client} I \mathrm{~d}=7511 \backslash \& \mathrm{RQT}=309 \backslash \& \mathrm{VName}=\mathrm{PQD}$

Hofstede, G. H. (2001). Culture's consequences: comparing values, behaviors, institutions, and organisations across nations. Sage.

Holloway, I., \& Todres, L. (2003). The status of method: Flexibility, consistency and coherence. Qualitative Research, 3, 345.

Howarth, C. (2002, June). Identity in whose eyes? the role of representations in identity construction. Journal for the Theory of Social Behaviour, 32, 145-162. Available from http://www.blackwell-synergy .com/doi/abs/10.1111/1468-5914.00181

Huguet, P., Latan, B., \& Bourgeois, M. (1998). The emergence of a social representation of human rights via interpersonal communication: empirical evidence for the convergence of two theories. European Journal of Social Psychology, 28, 831-846. Available from http : / / dx.doi.org/10.1002/(SICI) 1099-0992 (199809/10)

Jamar, S. D. (1992). The protection of intellectual property under islamic law. Capital University Law Review, 21, 1079-1106. 
Jost, J. T., \& Kruglanski, A. W. (2002). The estrangement of social constructionism and experimental social psychology: History of the rift and prospects for reconciliation. Personality and Social Psychology Review, 6, 168-187. Available from http://psr.sagepub.com/cgi/ content/abstract/6/3/168

Kapczynski, A., Chaifetz, S., Katz, Z., \& Benkler, Y. (2005). Addressing global health inequities: An open licensing approach for university innovations. Berkeley Technology Law Journal, 20, 1031.

Khan, Z. (2002). Intellectual property and economic development: Lessons from american and european history. Lodon: British Commission on Intellectual Property Rights. Available from http: //www.iprcommission.org/papers/pdfs/study _papers/spla_khan_study.pdf

Kuran, T. (2001). The provision of public goods under islamic law: Origins, impact, and limitations of the waqf system. Law $\mathcal{E}$ Society Review, 35, 841-898. Available from http://links.jstor.org/sici?sici=0023-9216\%282001\% $2935 \div 3 A 4 \div 3 \mathrm{C} 841 \% 3 \mathrm{ATPOPGU} \div 3 \mathrm{E} 2.0 . \mathrm{CO} \div 3 \mathrm{~B} 2-\mathrm{Y}$

Kurzweil, R. (2000). The age of spiritual machines: When computers exceed human intelligence. Penguin (Non-Classics).

Lakoff, G., \& Johnson, M. (1980a, August). Conceptual metaphor in everyday language. The Journal of Philosophy, 77, 453-486. Available from http://links.jstor.org/sici?sici=0022-362x\% $28198008 \div 2977 \div 3 \mathrm{~A} 8 \% 3 \mathrm{C} 453 \div 3 \mathrm{ACMIEL} \div 3 \mathrm{E} 2.0 . \mathrm{CO} \div 3 \mathrm{~B} 2-\mathrm{S}$

Lakoff, G., \& Johnson, M. (1980b). The metaphorical structure of the human conceptual system. Cognitive Science: A Multidisciplinary Journal, 4, 195-208. Available from http: / / www. informaworld. com/ $10.1207 /$ s15516709cog0402_4

Lakoff, G., \& Johnson, M. (1980c). Metaphors we live by (2nd ed.). University Of Chicago Press.

Lessig, L. (2001). The future of ideas: The fate of the commons in a connected world. Random House.

Levin-Rozalis, M. (2007). Playing by the rules: Social representations of 'law' as the socio-cognitive mediating mechanism between law and 
society. Theory Psychology, 17, 5-31. Available from http://tap . sagepub.com/cgi/content/abstract/17/1/5

Liu, J. H., Goldstein-Hawes, R., Hilton, D., Huang, L.-L., GastardoConaco, C., Dresler-Hawke, E., et al. (2005). Social Representations of Events and People in World History Across 12 Cultures. Journal of Cross-Cultural Psychology, 36(2), 171-191. Available from http: / / jcc.sagepub.com/cgi/content/abstract/36/2/171

Liu, J. H., \& Sibley, C. (2006). Differential effects of societal anchoring and attitude certainty in determining support or opposition to (bi)cultural diversity in new zealand. Papers on Social Representations, 15, 1.1-1.15. Available from http: / / www.psr.jku.at/

Liu, J. H., Wilson, M. S., McClure, J., \& Higgins, T. R. (1999). Social identity and the perception of history: cultural representations of aotearoa/new zealand. European Journal of Social Psychology, 29, 10211047. Available from http://dx.doi.org/10.1002/(SICI) 1099-0992 (199912) 29:81021: :AID-EJSP 9753.0.CO;2-4

Liu, L. (2004). Sensitising concept, themata and shareness: A dialogical perspective of social representations. Journal for the Theory of Social Behaviour, 34, 249-264. Available from http://www.blackwell-synergy.com/doi/abs/10.1111/ j.0021-8308.2004.00247.x

Liu, L. (2006). Quality of life as a social representation in china: A qualitative study. Social Indicators Research, 75, 217-240. Available from http://dx.doi.org/10.1007/s11205-004-3198-z

Locke, J. (1838). An essay concerning human understanding. printed by Thomas Davison.

Markov, I. (2003). Dialogicality and social representations: The dynamics of mind. Cambridge UniversityPress.

Markova, I. (1996). Towards an epistemology of social representations. Journal for the Theory of Social Behaviour, 26, 177-196.

Markova, I. (2000). Amedee or how to get rid of it: social representations from a dialogical perspective. Culture and Psychology; 6 (4) Dec 2000.

Matsumura, S. (2006). Orangutan sociality, tool use, and human culture (van schaik's among orangutans) among orangutans: Red apes and 
the rise of human culture . by carel van schaik . cambridge: Belknap press of harvard university press, 2004. Current Anthropology, 47, 878-882. Available from http://www.journals.uchicago .edu/doi/abs/10.1086/507191

McGraw, A. P., \& Tetlock, P. E. (2005). Taboo trade-offs, relational framing, and the acceptability of exchanges. Journal of Consumer Psychology, 15, 2-15. Available from http://www.sciencedirect.com/science/article/B8JGB -4RNT6WS-2/2/6de04bdb244a2cdb4 7089c03c0eae270

McGuire, W. J. . (1986). The vicissitudes of attitudes and similar representational constructs in twentieth century psychology. European Journal of Social Psychology. Vol 16(2).

Moloney, G., Hall, R., \& Walker, I. (2005, September). Social representations and themata: The construction and functioning of social knowledge about donation and transplantation. British Journal of Social Psychology, 44, 415-441. Available from http://www. ingentaconnect.com/content/bpsoc/bjsp/ 2005/00000044/00000003/art00006

Moloney, G., \& Walker, I. (2002). Talking about transplants: social representations and the dialectical, dilemmatic nature of organ donation and transplantation. British Journal of Social Psychology; 41 (2) Jun 2002.

Moscovici, S. (1984). The phenomenon of social representations. , 3-69.

Moscovici, S. (1988). Notes towards a description of social representations. Journal of European Social Psychology, 18, 211-250;.

Moscovici, S. (2001). Why a theory of social representation?

Moscovici, S., \& Markova, I. (1998). Presenting social representations: A conversation. Culture Psychology, 4, 371-410. Available from http://cap.sagepub.com/cgi/content/abstract/4/ $3 / 371$

Moscovici, S., \& Vignaux, G. (1994). Le concept de themata (Structures et Transformations de Representations Sociales ed.; C. Guimelli, Ed.). Neuchatel: Delachaux et Niestle. (Translated article in published book) 
Nachbar, T. (2006). Monopoly, mercantilism, and intellectual property. Virginia Law Review, May. Available from http:// repositories .cdlib.org/bclt/lts/9/

On islamic customs and "ip": Jakarta post. (n.d.). Available from http://osdir.com/ml/org.dmca-activists/2005-10/ msg00007.html

Pawlowski, S. D., \& Nenov, I. N. (2004). It offshoring in the professional media (1999-2003): a social representations analysis. In (p. 81-83). Tucson, AZ, USA: ACM.

Perelman, M. (2003). The political economy of intellectual property. Monthly Review, 54. Available from http: / / www . monthlyreview .org/0103perelman.htm

Poeschl, G. (2004). Dinâmicas posicionais e dinâmicas representacionais: Reflexões e interrgações. / positional dynamics and representational dynamics: Thoughts and interrogations. Psicologia: Revista da Associação Portuguesa Psicologia. Vol 18(1).

Potter, J., \& Billig, M. (1992). Re-representing representations discussion of räty \& snellman. Papers on Social Representations, 1, 15-20. Available from http://www.psr.jku.at/PSR1992/ abstracts1992/1_15abstr.html

Potter, J., \& Edwards. (1999). Social representations and discursive psychology: From cognition to action. Culture and Psychology, 5, 447-458. Available from http://209.85.173.104/search?q=cache: wE4UGkahDrs J:Www-staff. 1boro.ac.uk/\{ \} ssde/Potter -Edwards\%2520Soc\%2520Reps\%26DP 2520CultPsy1999 - pdf+brute+facts+2B+social+representations $\backslash \& h l=$ en $\backslash \& c t=c l n k \backslash \& c d=9 \backslash \& g l=n z \backslash \& c l i e n t=$ firefox $-\mathrm{a}$

Potter, J., \& Wetherell, M. (1987). Discourse and social psychology: Beyond attitudes and behaviour. SAGE.

Pratchett, T., Stewart, I., \& Cohen, J. S. (2003). Science of discworld ii: The globe: 2 (New Ed ed.). Ebury Press.

Public participation in patent examination - wikipedia, the free encyclopedia. (n.d.). Available from http://en.wikipedia.org/wiki/ Public_participation_in_patent_examination 
Pugatch, M. P., \& Elgar, E. (2006). The intellectual property debate: Perspectives from law, economics and political economy. Edward Elgar Publishing.

Robinson, W. P. (1996). Social groups and identities: Developing the legacy of henri tajfel. Routledge.

Rose, D., Efraim, D., Gervais, M., Joffe, H., Jovchelovitch, S., \& Morant, N. (1995). Questioning consensus in social epresentation theory. Papers on Social Representations, 4, 150-176.

Rose, M. (1995). To steal a book is an elegant offense: Intellectual property law in chinese civilization. Business History Review, 69, 448. Available from http://proquest.umi.com/pqdweb?did= 9622909 \\&Ft=7\\&client $I d=7511 \backslash \& R Q T=309 \backslash \& V N a m e=P Q D$

Rose, M. (1996). Mothers and authors: Johnson v. calvert and the new children of our imaginations. Critical Inquiry, 22, 613. Available from http: / /proquest . umi.com/pqdweb?did=10086976\\&Fmt= $7 \backslash \& \mathrm{Client} I \mathrm{~d}=7511 \backslash \& \mathrm{R} Q \mathrm{~T}=309 \backslash \& \mathrm{VName}=\mathrm{P} Q \mathrm{D}$

Rose, M. (2002a, October). Copyright and its metaphors. UCLA Law Review, 50, 1 .

Rose, M. (2002b). Pragmatic plagiarism: Authorship, profit, and power. Comparative Literature, 54, 270. Available from http://proquest. umi.com/pqdweb?did=200147891\\&Fmt= $7 \backslash \& \mathrm{Client} I d=7511 \backslash \& \mathrm{R} Q \mathrm{~T}=309 \backslash \& \mathrm{VName}=\mathrm{P} Q \mathrm{D}$

Rose, M. (2003). Nine-tenths of the law: The english copyright debates and the rhetoric of the public domain. Law and Contemporary Problems, 66, 75. Available from http://proquest.umi.com/ pqdweb?did=337281761 \\&Fmt=7\\&client Id=7511 \\&RQT= $309 \backslash \& \mathrm{VName}=\mathrm{PQD}$

Ross, M. (2005). Briefing paper to united nations special rapporteur on the wai 262 claims to indigenous flora and fauna me o o ratou taonga katoa. Available from www.pcpd.org.nz/sr/wai262.pdf

Ruttan, L. (2006). Sociocultural heterogeneity and the commons. Current Anthropology, 47, 843-853. Available from http://www. journals .uchicago.edu/doi/abs/10.1086/507185

Räty, H., \& Snellman, L. (1992). Making the unfamiliar familiar - some 
notes on the criticism of the theory of social representations. Papers on Social Representations, 1, 3-14. Available from http://www.psr -jku.at/PSR1992/abstracts1992/1_15abstr.html

Sacks, H., Schegloff, E. A., \& Jefferson, G. (1974, December). A simplest systematics for the organization of turn-taking for conversation. Language, 50, 696-735. Available from http://links.jstor.org/sici?sici=0097-8507\% $28197412 \div 2950 \div 3 \mathrm{~A} 4 \div 3 \mathrm{C} 696 \% 3 \mathrm{AASSFTO} \div 3 \mathrm{E} 2.0 . \mathrm{CO} \div 3 \mathrm{~B} 2-\mathrm{R}$

Sapir, E. (1963). Selected writings of edward sapir in language, culture and personality. Berkeley: Univ. of California Press.

Sapir-whorf hypothesis - wikipedia, the free encyclopedia. (n.d.). Available from http://en.wikipedia.org/wiki/Sapir_Whorf

Schecter, M., \& Murray, S. (2007). Peer to patent: Community patent review a project pilot in which readers should consider participating. Available from http://www.marketsandpatents.com/bulletin/ IPB-10152007.html

Shirky, C. (2008). Here comes everybody: The power of organizing without organizations. Penguin Press HC, The.

Sidanius, J., \& Pratto, F. (1999). Social dominance: An intergroup theory of social hierarchy and oppression. Cambridge University.

Spini, D., \& Doise, W. (2005). Universal rights and duties as normative social representations. Finkel, Norman J.; Moghaddam, Fathali M. (2005). The psychology of rights and duties: Empirical contributions and normative commentaires. The law and public policy. (pp.

Stallman, R. (2004). Did you say "intellectual property"? it's a seductive mirage. Available from http://www.gnu.org/philosophy/not -ipr.xhtml

Tajfel, H., \& Turner, J. C. (1986). The social identity theory of intergroup behavior. Psychology of Intergroup Relations, 2, 7-24.

Universal declaration of human rights - wikipedia, the free encyclopedia. (n.d.). Available from http://en.wikipedia.org/wiki/Universal _declaration_of_human_rights

Vaidhyanathan, S. (2001). Copyrights and copywrongs: The rise of intellectual property and how it threatens creativity. NYU Press. 
Verheggen, T., \& Baerveldt, C. (2007). We don't share! the social representation approach, enactivism and the ground for an intrinsically social psychology. Culture and Psychology, 5-27.

Verkuyten, M., \& Brug, P. (2004). Multiculturalism and group status: The role of ethnic identification, group essentialism and protestant ethic. European Journal of Social Psychology, 24, 647 - 661.

Voelklein, C., \& Howarth, C. (2005). A review of controversies about social representations theory: A british debate. Culture $\mathcal{E}$ Psychology, $11,431$.

Wagner, W. (1995). Social representations, group affiliation, and projection: Knowing the limits of validity. European Journal of Social Psychology, 25, 125-139. Available from http: / / dx. doi .org/10.1002/ejsp .2420250202

Wagner, W. (1998). Social representations and beyond: Brute facts, symbolic coping and domesticated worlds. Culture \& Psychology. Vol 4(3).

Wagner, W., Duveen, G., Farr, R., Jovchelovitch, S., Lorenzi-Cioldi, F., Markova, I., et al. (1999, April). Theory and method of social representations. Asian Journal Of Social Psychology, 2, 95125. Available from http://www.blackwell-synergy.com/ doi/abs/10.1111/1467-839x.00028

Wagner, W., \& Hayes, N. (2005). Everyday discourse and common sense: The theory of social representations. Palgrave Macmillan.

Wagner, W., Kronberger, N., \& Seifert, F. (2002, September). Collective symbolic coping with new technology: Knowledge, images and public discourse. The British journal of social psychology / the British Psychological Society, 41, 323-43. (PMID: 12419006)

Warner, J. (1999, April). Information society or cash nexus? a study of the united states as a copyright haven. Journal of the American Society for Information Science, 50, 461.

Wetherell, M. (1998). Positioning and interpretative repertoires: Conversation analysis and post-structuralism in dialogue. Discourse Society, 9, 387-412. Available from http://das.sagepub.com/cgi/ content/abstract/9/3/387

Yamagishi, T., \& Kiyonari, T. (2000, June). The group as the container of 
generalized reciprocity. Social Psychology Quarterly, 63, 116.

Yamagishi, T., Mifune, N., Liu, J., \& Pauling, J. W. (in press). Exchanges of group-based favors: Ingroup bias in the prisoner's dilemma game with minimal groups in japan and new zealand. Asian Journal of Social Psychology, 11, 31. Available from http: / / www.blackwell -synergy.com/toc/ajsp/10 (AJSP-07-060-RA.R2)

Yasuda, F., \& Kato. (2007). Measuring the economic impact of ip systems. Available from http: / /www.wipo.int/portal/en/news/ 2007 /article_0032.html

Yu, N. (1998). The contemporary theory of metaphor: A perspective from chinese. John BenjaminsPublishing Company.

Zimmer, C. (1995, November). Tooling through the trees. Discover, 16, 46. Available from http://proquest. umi.com/pqdweb? did= $8654521 \backslash \& \mathrm{Fmt}=7 \backslash \& \mathrm{Client} I \mathrm{~d}=7511 \backslash \& \mathrm{RQT}=309 \backslash \& \mathrm{VName}=\mathrm{PQD}$

Zimmer, C. (2007, November). From ants to people, an instinct to swarm. The New York Times. 